\title{
WestVirginiaUniversity
}

THE RESEARCH REPOSITORY @ WVU

Graduate Theses, Dissertations, and Problem Reports

2016

\section{Optical and Terahertz Measurements of Spintronic Materials}

Derek A. Bas

Follow this and additional works at: https://researchrepository.wvu.edu/etd

\section{Recommended Citation}

Bas, Derek A., "Optical and Terahertz Measurements of Spintronic Materials" (2016). Graduate Theses, Dissertations, and Problem Reports. 5161.

https://researchrepository.wvu.edu/etd/5161

This Dissertation is protected by copyright and/or related rights. It has been brought to you by the The Research Repository @ WVU with permission from the rights-holder(s). You are free to use this Dissertation in any way that is permitted by the copyright and related rights legislation that applies to your use. For other uses you must obtain permission from the rights-holder(s) directly, unless additional rights are indicated by a Creative Commons license in the record and/ or on the work itself. This Dissertation has been accepted for inclusion in WVU Graduate Theses, Dissertations, and Problem Reports collection by an authorized administrator of The Research Repository @ WVU.

For more information, please contact researchrepository@mail.wvu.edu. 


\title{
Optical and Terahertz Measurements of Spintronic Materials
}

\author{
Derek A. Bas
}
Dissertation submitted to the Eberly College of Arts and Sciences
at West Virginia University
in partial fulfillment of the requirements
for the degree of
Doctor of Philosophy
in
Physics

\author{
Alan D. Bristow, Ph.D., Chair \\ Mikel Holcomb, Ph.D. \\ David Lederman, Ph.D. \\ Tudor Stanescu, Ph.D. \\ Thomas E. Wilson, Ph.D. \\ Department of Physics and Astronomy \\ Morgantown, West Virginia \\ 2016
}

Keywords: nonlinear optics; ultrafast optics; terahertz time-domain spectroscopy; spintronics; antiferromagnetic magnons; topological insulator; manganese fluoride; bismuth selenide;

coherent control; ultrafast photocurrents

Copyright 2016 Derek A. Bas 


\title{
Abstract
}

\section{Optical and Terahertz Measurements of Spintronic Materials}

\author{
Derek A. Bas
}

Terahertz time-domain spectroscopy (THz-TDS) is a versatile method to determine lattice, electronic charge and spin dynamics. This dissertation employs THz-TDS to study the spin and charge dynamics in topological insulator and antiferromagnetic systems. Observing time-domain effects on the scale of picoseconds gives unprecedented control over optoelectronic properties. Methods and challenges of $\mathrm{THz}$ generation, detection, and transmission are outlined. The wealth of light-matter interactions present in all nonlinear optical experiments are discussed, including primarily optical rectification, shift currents, and injection currents. Each of these gives valuable insight into the carrier dynamics of a material type.

Conventional electronics can be improved in their speed and efficiency by taking advantage of an additional degree of freedom- electron spin. Therefore, we consider material types which exhibit great potential to replace common electronic materials while simultaneously employing electron spin for information storage or transport.

Antiferromagnets show a type of spin-order that has the ability to store bits without unwanted interactions between neighboring particles. In antiferromagnetic $\mathrm{MnF}_{2}$ which has a Néel temperature of $T_{N}=67 \mathrm{~K}, \mathrm{THz}$-TDS is performed on one-magnon and two-magnon resonances in the $0.1-2.3 \mathrm{THz}$ range while varying the temperature from 6 to $295 \mathrm{~K}$. The behavior of the one-magnon resonance is modeled by modified molecular field theory with an additional coupling term $j$ set as a free parameter to fit the data. The resulting best fit value $j=1.1$ provides the first experimental evidence indicating that neighboring spins in $\mathrm{MnF}_{2}$ are only weakly coupled, closely approximating mean-field theory. Time-of-flight analysis was performed on the transmitted $\mathrm{THz}$ pulses to measure the temperature-dependent $\mathrm{THz}$ refractive index, which was modeled by phonon energy in the $T>T_{N}$ regime and magnetic energy in the $T<T_{N}$ regime. In the range $T<10 \mathrm{~K}$, measured data deviates from this theory, and can be modeled by internal energy from hyperfine interactions, providing the first direct observation of hyperfine interactions in $\mathrm{THz}$ spectroscopy.

Topological insulators exhibit the ability to transport spin-polarized currents along their surfaces with high mobilities. Phase-related pulses at photon energies 0.8 and $1.6 \mathrm{eV}$ are used to simultaneously inject shift and injection currents into thin-films of the prototypical topological insulator $\mathrm{Bi}_{2} \mathrm{Se}_{3}$, and the foundation is laid out for an extensive study of the novel carrier properties in topological surface states. A method of symmetry analysis based on the crystal lattice is developed for isolation and individual study of the surfaceonly shift currents, which are threefold symmetric with equal components parallel and perpendicular to the pump polarization, and bulk/surface injection currents, which are isotropic parallel to the pump polarization and vanishing perpendicular to it. Pump energies can be tuned through the Dirac point, a capability which holds promise for the search of smoking gun evidence for the novel topological insulator surface state behavior that has been theorized. 


\section{Acknowledgments}

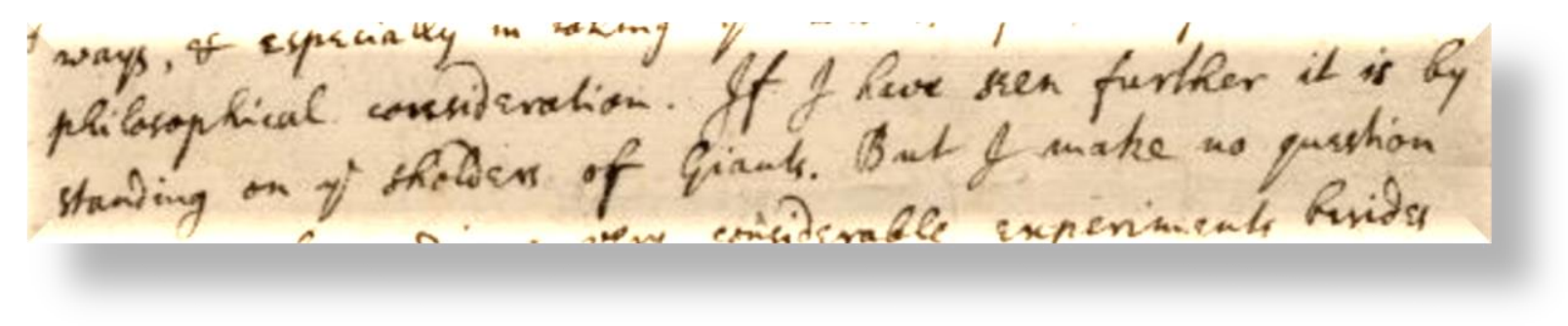

Isaac Newton used the old metaphor in his letter to Robert Hooke. The first time I saw the phrase "on the shoulders of giants" was on the cover of the Hawking book, and it immediately struck me as a profound concept, and one that holds great meaning to my scientific side. Scientific and technological progress is incredible to me, and far greater than any individual can ever hope to be. I hope my own contribution will be significant, but it was only possible through the work of my many predecessors. And so I sincerely thank the countless giants on whose shoulders I stand.

None of this work could have been accomplished without the sponsorship of the West Virginia Higher Education Policy Commission (HEPC.dsr.12.29) and the National Science Foundation (CBET-1233795). I also acknowledge the use of the WVU Shared Research Facilities.

l'd like to say thank you to my advisor, Dr. Bristow, for the years of patience and mentoring. To him I owe especially my care and attention to detail in the presentation of my research. And I thank the rest of my Ph.D. committee, Dr. Holcomb, Dr. Stanescu, Dr. Lederman, and Dr. Wilson, for helpful criticism and support, which aided in getting me to where I am today. And I thank my everyday colleagues as well, especially Joe Rowley, Brian Wilmer, Tess Senty, and Scott Cushing, for conversations and distractions to help me get through grad school. Thanks also to Kevin Vargas-Velez, Sercan Babakiray, Trent Johnson, Dr. Borisov, Rodrigo Muniz, and Dr. Sipe for the valuable input to our collaborations.

Of course, people outside my scientific community were valuable to my success as well. I cannot thank my family enough for their emotional support and their belief in me. And thanks, Chris, for making the long trip to watch me defend. And most of all I thank my wife Lis, who was there for me through the whole story, who didn't let me give up, and who made me want to always be the best possible version of myself.

-Derek Bas

http://digitallibrary.hsp.org/index.php/Detail/Object/Show/object_id/9285 


\section{Table of Contents}

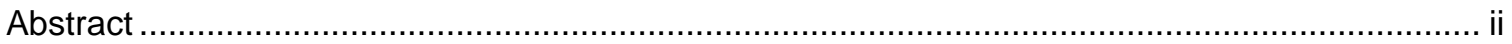

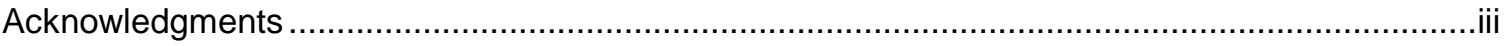

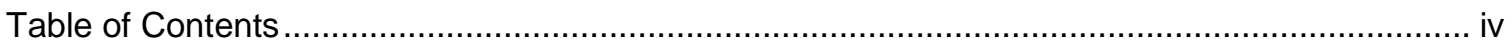

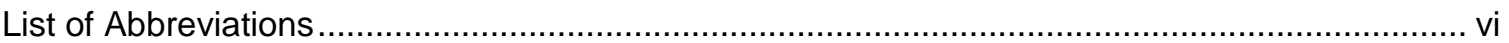

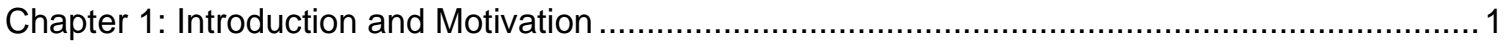

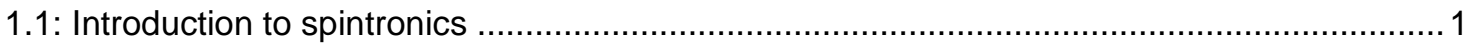

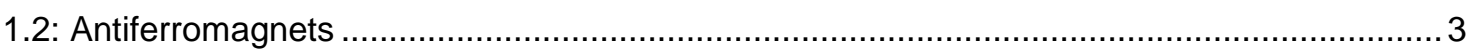

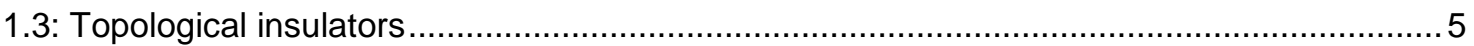

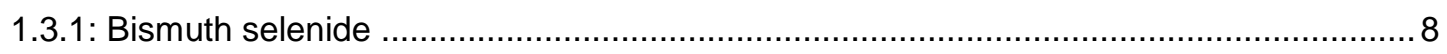

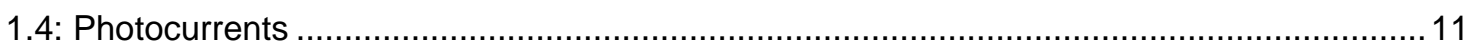

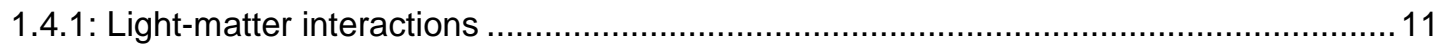

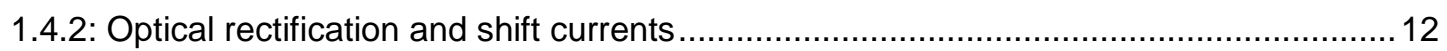

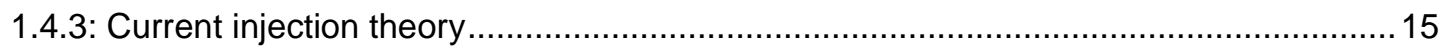

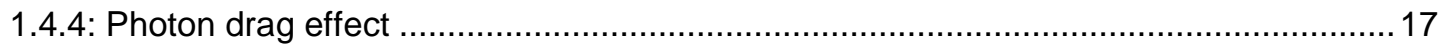

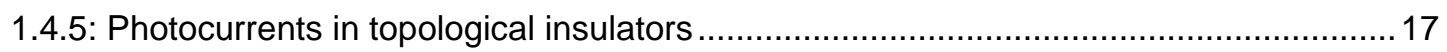

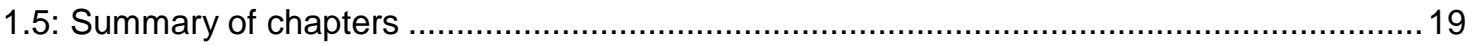

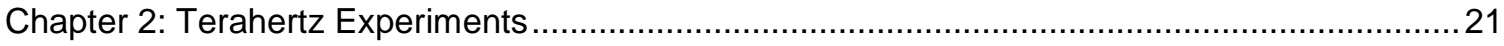

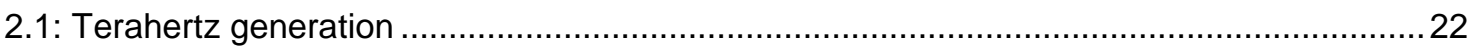

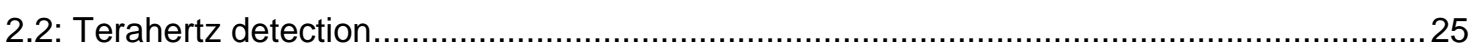

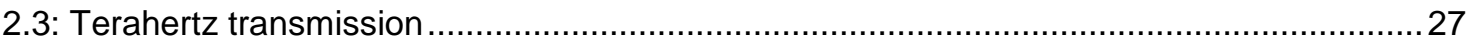

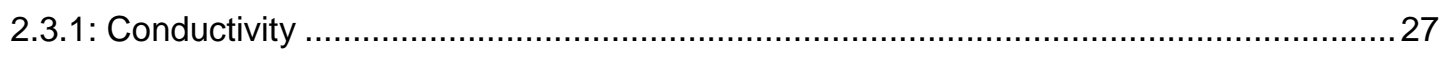

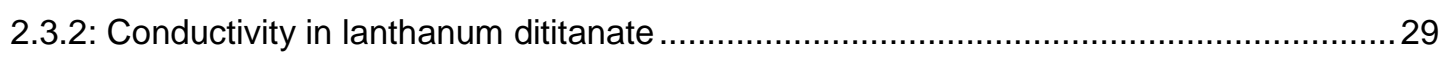

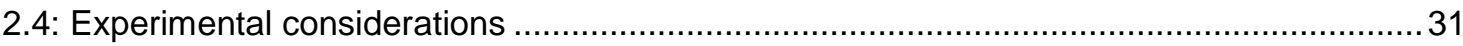

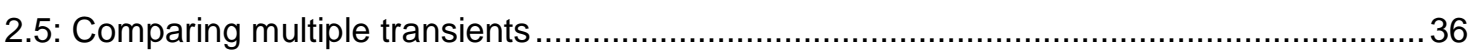

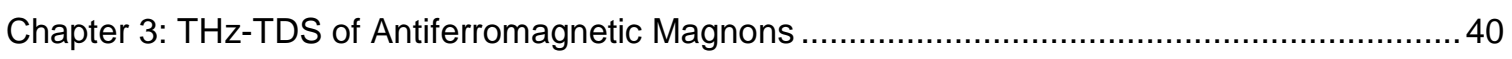

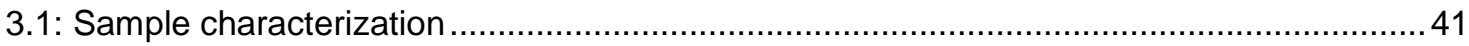

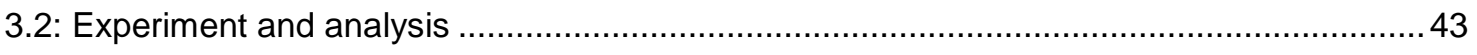

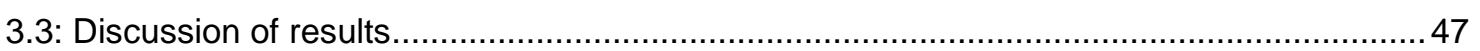

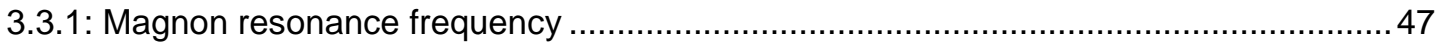

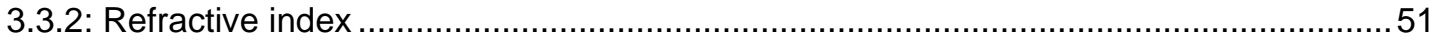

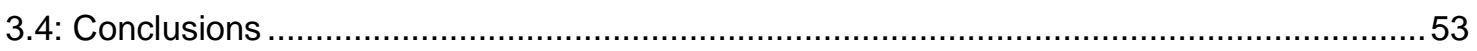




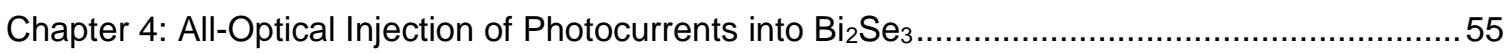

4.1: $\mathrm{Bi}_{2} \mathrm{Se}_{3}$ thin-film growth by molecular beam epitaxy .......................................................56

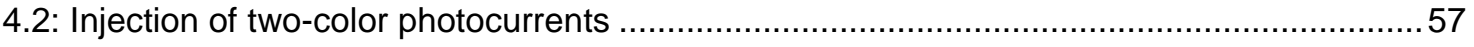

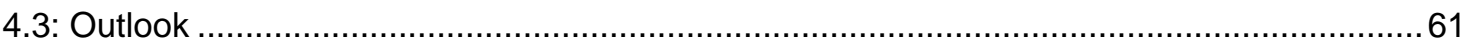

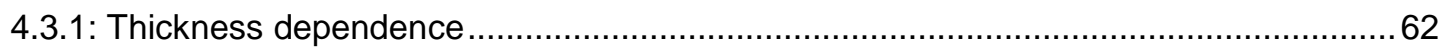

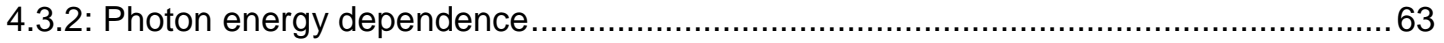

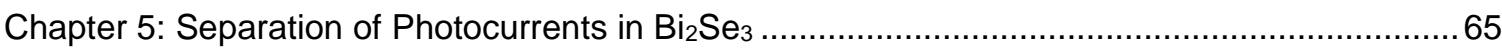

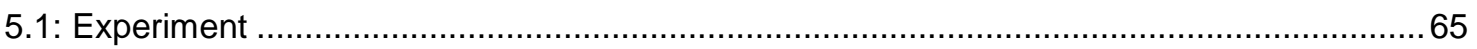

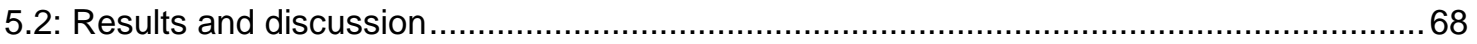

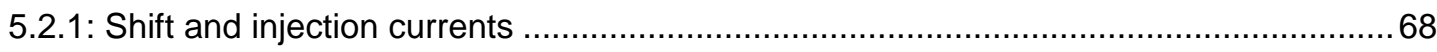

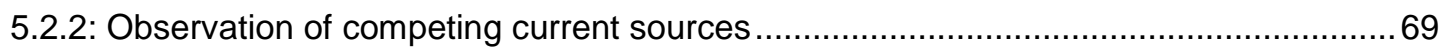

5.2.3: Separation of shift and injection currents ........................................................... 73

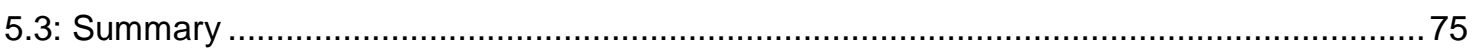

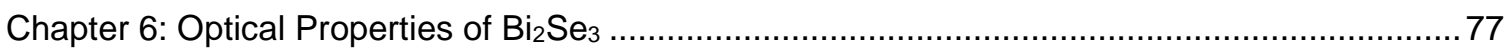

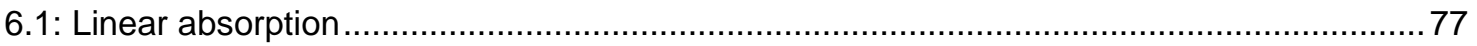

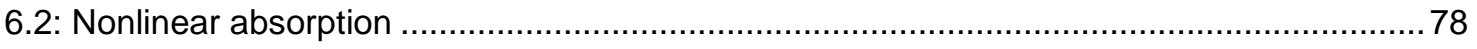

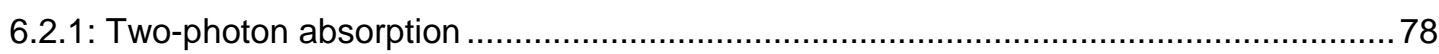

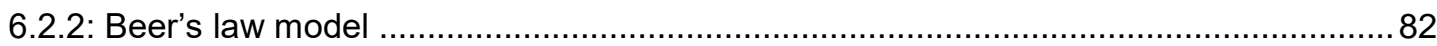

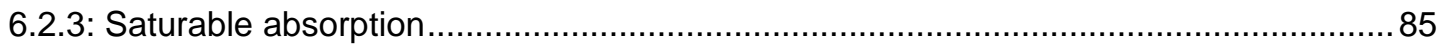

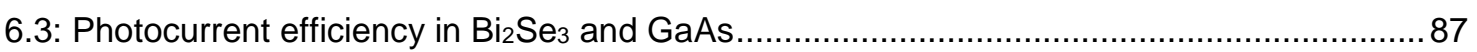

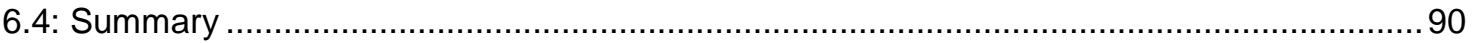

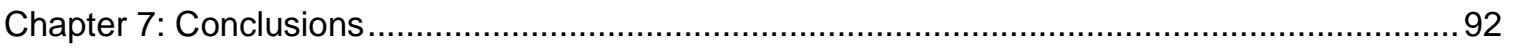

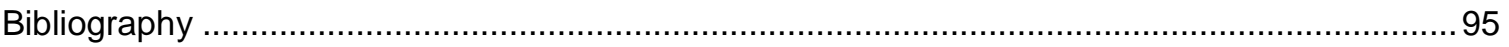

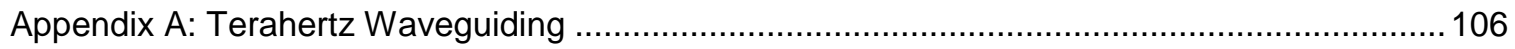

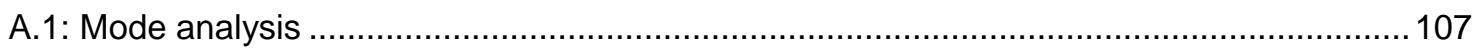

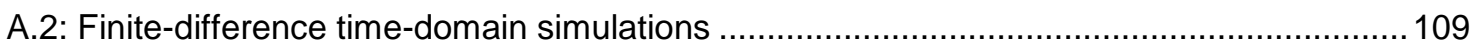

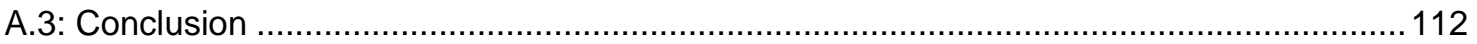




\section{List of Abbreviations}

\begin{tabular}{ll} 
1PA & Single-photon absorption \\
2PA & Two-photon absorption \\
3PA & Three-photon absorption \\
AF & Antiferromagnet / antiferromagnetic \\
CB & Conduction band \\
CW & Continuous wave \\
DOS & Density of states \\
EO & Electro-optic \\
IC & Injection current \\
IR & Infrared \\
ND & Neutral density \\
OR & Optical rectification \\
QL & Quintuple layer \\
SA & Saturable absorption / saturable absorber \\
SC & Shift current \\
SS & Surface states \\
THz & Terahertz \\
THz-TDS & Terahertz time-domain spectroscopy \\
TI & Topological insulator \\
VB & Valence band \\
\hline
\end{tabular}




\section{Chapter 1: Introduction and Motivation}

\section{1: Introduction to spintronics}

The emerging field of spintronics is the electron spin-based counterpart to conventional chargebased electronics. ${ }^{1}$ Instead of relying on the transport of charge, it relies on the transfer of spin, which can occur without moving charges at all. Spin transfer simply replaces charge transfer in some applications (providing advantages in speed, efficiency, and stability), and in others it can be thought of as an additional degree of freedom to add capability and performance. In the endless pursuit of technological advancement, the capability to understand and exploit electron spin gives countless new options for scientists and engineers to take advantage of.

A key challenge in the development of spintronics is the need to identify and characterize spinbased materials, and this effort is the major purpose of this thesis. Various types of materials are identified, some typical and some exotic, and work is performed leading towards a complete understanding of their electron spin structure. The fundamental research questions that must be explored here as well as in any spintronics work are the questions of how to create and detect a spin-polarization in a system, and how long the spin polarization will last in equilibrium conditions.

Some spintronics devices have already been proposed in other works. ${ }^{2}$ One example is a diode that operates by allowing a high current of electrons with spin $+1 / 2$, while suppressing the current of electrons with spin $-1 / 2$. Another is a spin transistor, in which the spin polarization is used as a switch to control the flow of current through the device. The fundamental premise of such devices is the Zeeman splitting inherent in magnetic materials. When a magnetic field $B$ is applied, energy levels that are otherwise degenerate become split by an energy

$$
\Delta E=g m_{j} \mu_{B} B
$$

Where $g$ is the Landé g-factor, $m_{j}$ is the total angular momentum, and $\mu_{B}$ is the Bohr magneton. This results in an overall equilibrium spin-polarization due to one spin being more energetically favorable than the opposite. 
The formation of an equilibrium spin order is the first hurdle in creating a spintronics device. Aside from Zeeman splitting, spin order can also arise through spin-orbit coupling (the interaction between the magnetic fields created by the orbital motion and spin of the same electrons) or exchange energy (the interaction allowing neighboring electrons to exchange positions). In materials such as antiferromagnets (AFs) and topological insulators (TIs), the spin polarization is a direct consequence of the material properties, and spin-orbit coupling and exchange energies play major roles in determining the degree of spin polarization at equilibrium.
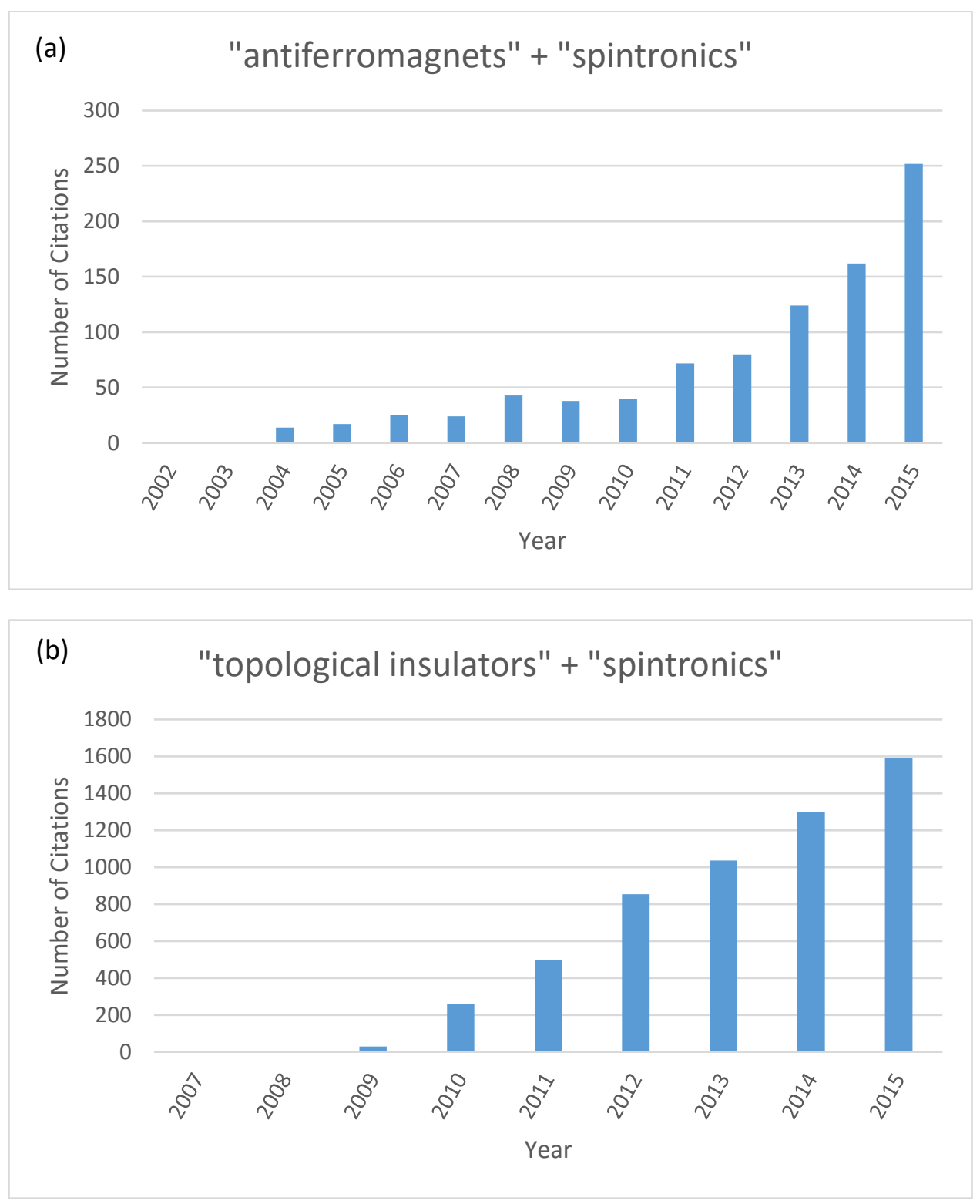

Figure 1.1: Citation report of spintronics topics generated by Web of Science. 
Although the foundations for spintronics were laid as early as the 1930s, the term "spintronics" was not used until the late 1990s. Figure 1.1 is a citation report using Web of Science illustrating the year-byyear prevalence of the terms "antiferromagnets spintronics" and "topological insulators spintronics" within citations of peer-reviewed articles. In the figure, (a) shows the recent interest in AF materials in the context of spintronics, and (b) shows that Tls are even more highly cited at present, depite being a newer addition to the field. Both classes of materials show great promise and perhaps in the near future they will be commonly used materials in everyday devices. But for the time being, there are many unanswered questions and it is important to determine which materials of each type are the most efficient at spinpolarization applications, and how they may be utilized and improved.

\section{2: Antiferromagnets}

AFs are desirable spintronic materials because of their magnetic structure and capability to store and transport spin while exhibiting zero macroscopic magnetization. AF devices may store information in the form of electron spin "bits", while remaining insensitive to external fields and nearby magnetic devices. To explain antiferromagnetism, the behavior of unpaired electrons in crystal lattices must be understood, namely in terms of their spin and the Pauli exclusion principle. Since electron spin is a quantum mechanical concept, magnetism cannot be explained using classical mechanics. Materials with unpaired electrons always exhibit some type of magnetism, which may be ferromagnetism, antiferromagnetism, or ferrimagnetism.

Two neighboring electrons orbiting adjacent ions in a crystal lattice may have spins that are either parallel or antiparallel to each other. If they are parallel, they cannot occupy the same space due to the Pauli exclusion principle. Being farther apart, they experience less Coulomb repulsion, and hence the system has a lower energy. Since electrons prefer to be in the lowest energy state possible, this leads to a material being ferromagnetic: In the presence of an influential field from either crystal asymmetry or an external source, all the unpaired electrons will tend to align along the same direction.

In some materials, neighboring magnetic ions are intermediated with nonmagnetic ions. This is the case in $\mathrm{MnO}$, which is made up of alternating $\mathrm{Mn}^{2+}$ ions and $\mathrm{O}^{2-}$ ions. Each $\mathrm{Mn}^{2+}$ ion has 5 unpaired 
electrons in its outer d orbitals, and because of Hund's first rule they must either be all spin up, or all spin down. Connecting neighboring $\mathrm{Mn}^{2+}$ ions is the outer $\mathrm{p}$ orbital from an $\mathrm{O}^{2-}$ ion, which is filled with two electrons- one spin up and one spin down. In order to covalently bond with its neighbors, the $\mathrm{O}^{2-}$ must donate a spin-up electron to one side and a spin-down to the other. The only way this can happen is for the first $\mathrm{Mn}^{2+}$ to be spin-down and the second to be spin-up. Therefore, it is energetically favorable for alternating $\mathrm{Mn}^{2+}$ ions to have antiparallel spin orientations. This phenomenon is called superexchange and it causes materials to be antiferromagnetic.

These two possibilities are simplified examples. The $\mathrm{O}^{2-}$ in the superexchange example could instead couple $\mathrm{Mn}$ ions of different valence. In this case, the $\mathrm{O}$ can donate to one $\mathrm{Mn}$, and the second $\mathrm{Mn}$ can then donate to the $\mathrm{O}$, essentially inverting the bond in space. But this can only happen if the $\mathrm{Mn}$ ions have parallel spins, and this possibility is energetically favorable because allowing electrons to hop reduces the kinetic energy. This is called double exchange, and it leads to ferromagnetism.

Even more complex examples exist, like additional intermediary atoms or nearest neighbors being coupled through superexchange at $90^{\circ}$ from each other. If this leads to antiparallel spins only partially cancelling each other, resulting in a small net magnetization, then the material is known as ferrimagnetic, but this possibility is not explored thoroughly in this work. The type of atoms involved, their proximity to each other, and their orientation all play a role in determining whether a material is ferromagnetic, antiferromagnetic, or ferrimagnetic.

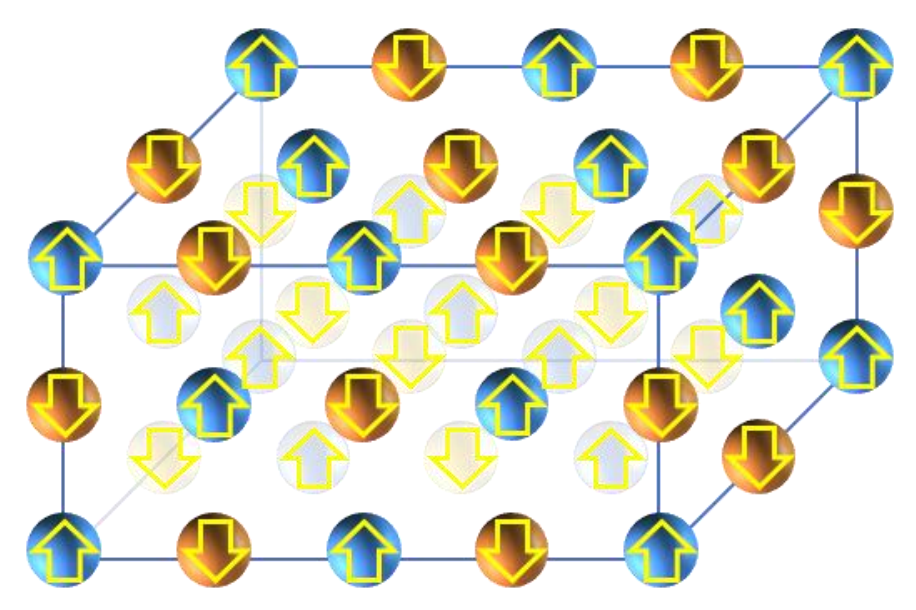

Figure 1.2: Schematic depicting an example of AF spin order. 
Materials often have a characteristic temperature at which they undergo significant state changes, which may be the melting point or boiling point. The magnetic counterpart in a ferromagnet is the Curie temperature, at which a transition occurs from a permanent magnet (spins remaining aligned in a particular direction even at equilibrium) to having spins only aligned in response to an external force (paramagnetic behavior). For an AF, this transition temperature is known as the Néel temperature $T_{N}$. When the material's crystal lattice reaches $T_{N}$, thermal energy from phonons in the lattice adds disorder to the system, randomizing the local spins, and at higher temperatures the material becomes paramagnetic. This means spins may still be aligned in an ordered fashion, but only in the presence of an external field. The AF studied in this work is manganese fluoride $\left(\mathrm{MnF}_{2}\right)$, in which the manganese atoms have unpaired spins that lend themselves to the spin order in the material.

The alternating spin structure in AFs is well-understood and has been first observed by neutron diffraction, ${ }^{3}$ in which neutron beams diffract from a lattice with constructive interference peaks which reveal the crystal symmetry much like in x-ray diffraction. Unlike x-rays, neutrons have a magnetic moment, so they diffract differently from spin-up and spin-down electrons, and thus can directly reveal the spin structure. But while AFs have been well-studied for decades, their interactions with light have only recently come under scrutiny. The ability to fully characterize AF materials and their associated magnon interactions using all-optical ultrafast techniques is valuable and therefore Chapter 3 will lay the foundation with $\mathrm{MnF}_{2}$ representing the prototypical example which can be extended to more complex material systems.

\section{3: Topological insulators}

The prediction and discovery of Tls comes from a 30+ year effort on the study of the quantum Hall effect. The quantum Hall effect occurs in a two-dimensional electron system with a strong applied magnetic field. The Lorentz force causes electrons to oscillate in orbits around the magnetic field vector in a similar manner to the classical Hall effect, with cyclotron frequency $v_{c}$ leading to quantized energy levels $E_{m}=$

$h v_{c}\left(m+\frac{1}{2}\right)$. These energy levels are called Landau levels, and they can be viewed as a band structure. If the system is cold enough, and the magnetic field is strong enough, then the Fermi energy will lie in between 
Landau levels, with an energy gap in between occupied and unoccupied Landau levels. In this case the system must be treated quantum mechanically, and the Hall conductance can only take on quantized values

$$
\sigma=\frac{n e^{2}}{h}
$$

where $e$ is the elementary charge, $h$ is Planck's constant, and $n$ is the integer filling factor. ${ }^{4,5}$

The quantization of the Hall conductance was observed by von Klitzing et al. in $1980^{6}$ using the two-dimensional electron gas in a metal-oxide-semiconductor field-effect transistor under the influence of a 15-T magnetic field. Due to the Hall conductance being forced to take on only well-defined, fixed values, the experiment was used as a highly accurate new means of calculating the fine-structure constant $\alpha$ to an accuracy of one part per billion. This precision also led to a new standard in calibration of resistance, based on what is now known as the von Klitzing constant $R_{K}=h / e^{2}$.

At the interface of a quantum Hall state and vacuum the topology changes, leading to edge states. ${ }^{7}$ The cyclotron orbits of the quantum Hall state meet the edge and are forced to continue on rather than complete their orbits. While the circular orbits create Landau levels which are similar to the energy levels in an ordinary insulator, the existence of the broken topology creates new gapless states which allow electrons to travel in one direction freely along the edge.

To understand the band structure of a $\mathrm{TI}$, one can look first to the simpler band structure of graphene. Graphene is a two-dimensional sheet of carbon atoms bonded together in a hexagonal lattice. The energy $E$ of electrons in graphene can be described by the dispersion relation

$$
E= \pm \sqrt{\gamma_{0}^{2}\left(1+4 \cos ^{2} \frac{k_{y} a}{2}+4 \cos \frac{k_{y} a}{2} \cdot \cos \frac{k_{x} \sqrt{3} a}{2}\right)}
$$

where $k_{x, y}$ is the momentum of electrons and $a \approx 2.46 \AA$ is the lattice parameter. This dispersion results in the electrons having a linear dispersion near the Dirac point, and the conduction band $(\mathrm{CB})$ meets the valence band (VB), forming a cone like the schematic in Figure 1.3. Thus, the electrons and holes behave as massless Dirac fermions. 
In a three-dimensional TI, a similar band structure occurs, but it only applies to charge carriers on the surfaces of the material. Since the VB meets the $C B$ in this cone, it represents metallic surface states (SS). The surfaces act as two-dimensional sheets of free Dirac fermions which behave relativistically. These carriers have a high conductivity in two dimensions, so in an ideal $\mathrm{TI}$, electrons flow freely along the surfaces. This behavior only occurs on the surfaces, as it comes from the breaking of the topology, analogous to the edge states created by the interface of a quantum Hall insulator. The bulk of the material has an uninteresting band structure similar to that of an ordinary insulator, where electrons remain trapped as they are in the Landau levels in a quantum Hall insulator.

(a)

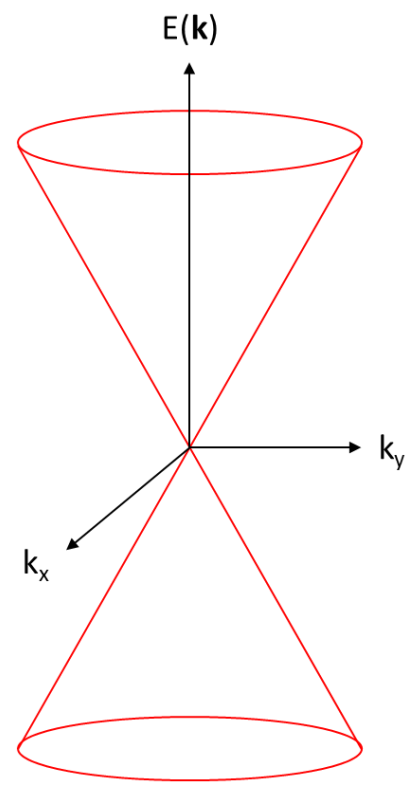

(b)

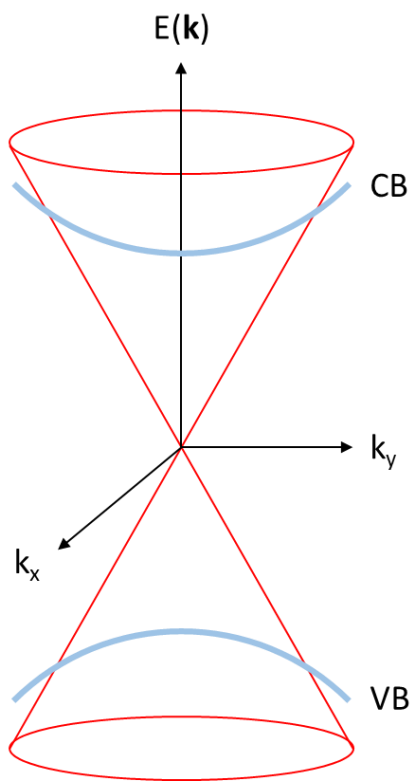

Figure 1.3: (a) Schematic of a Dirac cone band structure, similar to the six symmetric cones at the $K$-points in graphene. (b) Schematic showing the Dirac cone in the SS of a TI, overlapped with the bulk VB and CB which are simultaneously present.

In addition to this dichotomy between bulk and SS electrons, the SS electrons share another interesting feature. Although they conduct freely, their spin interacts strongly with the outer layer of atoms because of how massive those atoms tend to be. Thus, they experience much stronger spin-orbit coupling than typical materials. The electron orbits produce a magnetic field that is strong enough to influence the spin of the electrons themselves. Therefore, the direction of the current is tied inextricably to the orbital direction of motion, and thus the momentum is physically locked orthogonally to the direction of electron 
spin. In other words, electrons traveling in one direction are forced to be spin-up, while electrons traveling in the opposite direction are forced to be spin-down. This behavior is illustrated in Figure 1.4.

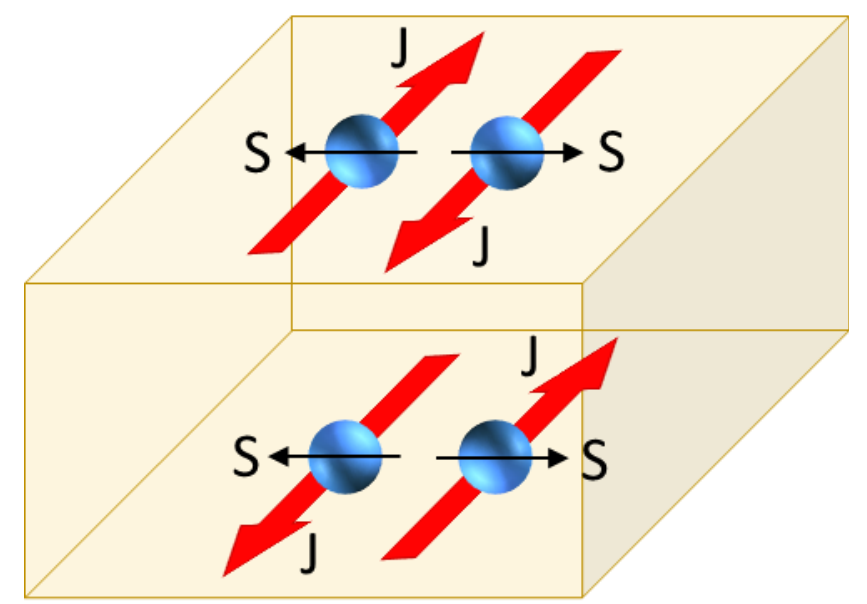

Figure 1.4: Spin-locking property of TI SS electrons (blue spheres). Red arrows show the momentum $J$ and black arrows show the spin $S$.

This spin-locking is the main property that makes Tls very interesting for spintronic applications. Using a TI, you can be sure that you know the spin-polarization of a current based on the direction in which it is traveling. And furthermore, the spin-polarized surface currents are protected by time-reversal symmetry, making them immune to backscatter by nonmagnetic impurities. ${ }^{8-11}$ This and the exceptional mobilities associated with the Dirac cone make the spin-polarized currents an exceptionally advantageous feature of Tls, making them promising components for future spintronic devices.

\subsection{1: Bismuth selenide}

Bismuth selenide $\left(\mathrm{Bi}_{2} \mathrm{Se}_{3}\right)$ has many reasons to generate interest among varying fields in physics. Its simple band structure and band gap of about $0.3 \mathrm{eV}$ (the largest amongst the common TIs) make it a potentially useful semiconductor for an extensive variety of applications. As the prototypical TI, it has been intensively studied for its electronic, thermoelectric, ${ }^{12,13}$ and optical properties. ${ }^{14-22}$ Among other things, it has been demonstrated to be an effective saturable absorber (SA) material useful for fiber lasers, ${ }^{23,24}$ a component in negative index of refraction metamaterials based on the tunability of its dielectric constant as a function of temperature and pressure, ${ }^{25}$ an efficient thermal energy harvester due to a high Seebeck 
coefficient, ${ }^{26}$ and an efficient light-to-heat converter for use in cancer cell treatment and diagnostics, ${ }^{27}$ applications which highlight the importance of the material's continued study and characterization.

$\mathrm{Bi}_{2} \mathrm{Se}_{3}$ is made up of a hexagonal lattice exhibiting bulk inversion symmetry with alternating layers of $\mathrm{Bi}$ and $\mathrm{Se}$ as shown in Figure 1.5. It naturally grows in increments of quintuple layers (QLs), each of which is made up of 5 atomic layers, and is approximately $1 \mathrm{~nm}$ thick. As with all thin films, additional layers result in a higher density of energy states, until the VB and CB become quasi continuous in thicker samples.

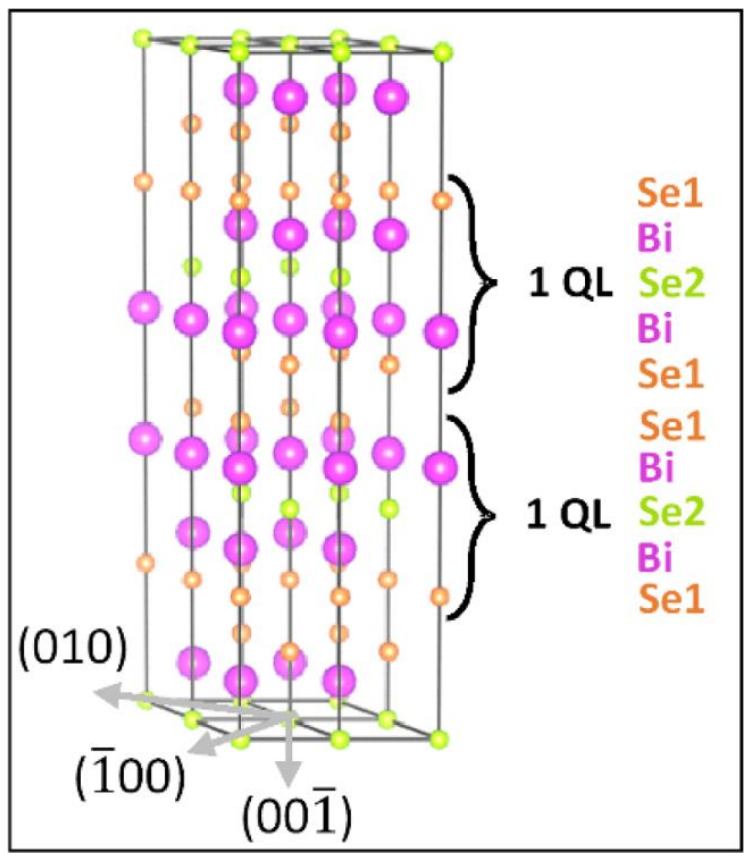

Figure 1.5: Rhombohedral atomic structure of $\mathrm{Bi}_{2} \mathrm{Se}_{3}$. A QL is approximately $1 \mathrm{~nm}$ thick. Purple spheres are $\mathrm{Bi}$ atoms, and orange and green spheres are Se atoms taking two different positions in terms of their symmetry.

Figure 1.6 shows the band structure of $\mathrm{Bi}_{2} \mathrm{Se}_{3}$ near the $\Gamma$-point, generated by an effective tightbinding model on a triangular lattice with eight bands per QL. ${ }^{11,28}$ The band indices from the bottom (VB) to the highest CB are $\alpha=1,2,3,4$ respectively, and each is divided into spins $\sigma=\uparrow, \downarrow$. Interactions between bands exist, but in the region near $k=0$ shown in the figure they are irrelevant. Unlike in graphene, $\mathrm{Bi}_{2} \mathrm{Se}_{3}$ has only one low-energy Dirac cone (red in the figure) and it is near the $\Gamma$ point. A second Dirac cone exists, but it is at higher energy with the same momentum as the first (blue). 


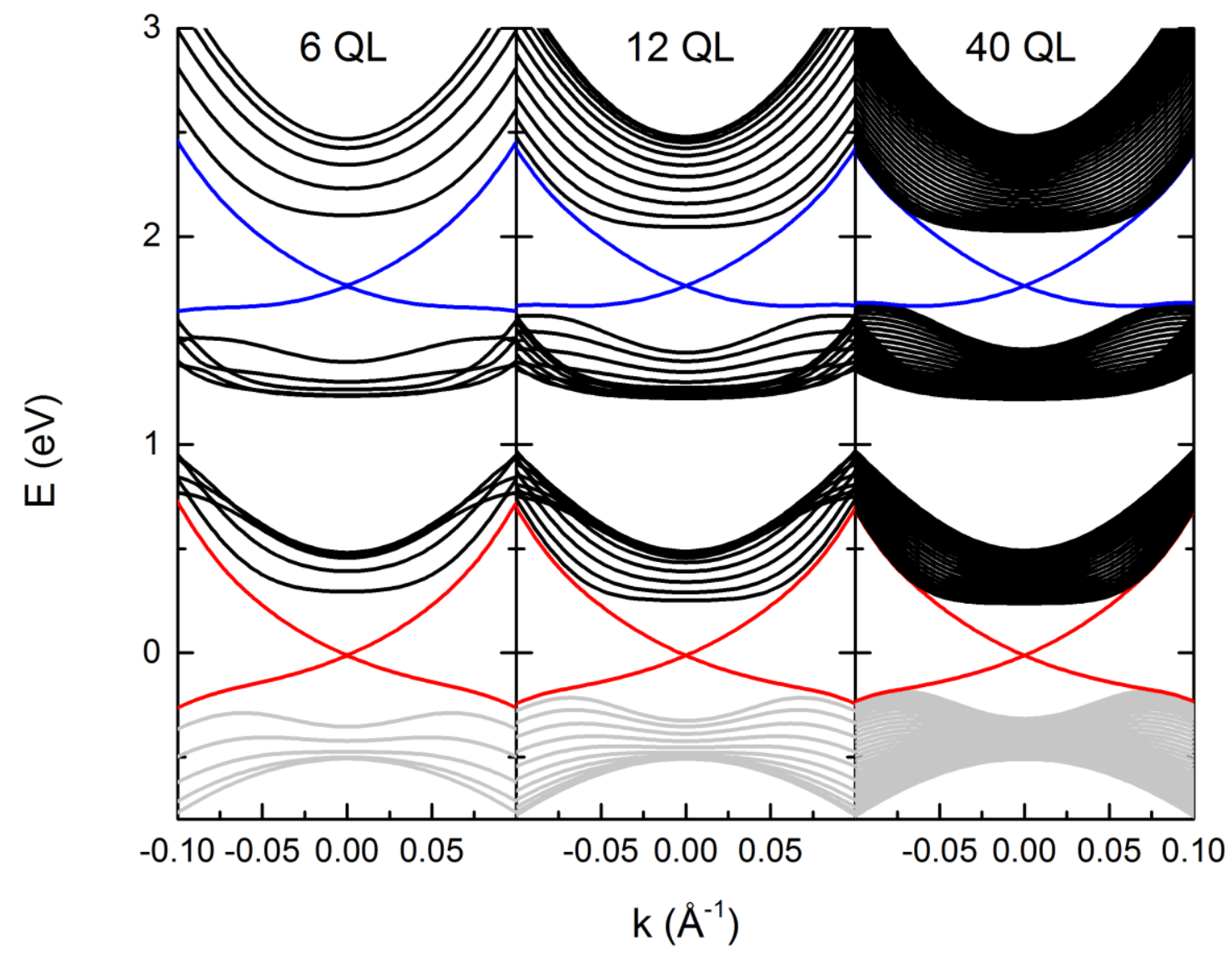

Figure 1.6: Band structure of $\mathrm{Bi}_{2} \mathrm{Se}_{3}$ films of thickness $6 \mathrm{QL}$ (left), $12 \mathrm{QL}$ (middle), and $40 \mathrm{QL}$ (right). Gray curves are VB, black curves are CB, red curves are the first set of SS, and blue curves are the second set of SS (SS2). As-grown samples tend to have a Fermi level somewhere inside the first set of $\mathrm{CB}$ states $(\sim 0.3 \mathrm{eV}$ on this scale).

Bismuth, being an exceptionally massive atom, provides strong spin-orbit coupling for the lattice. This gives $\mathrm{Bi}_{2} \mathrm{Se}_{3}$ its unique topologically protected $\mathrm{SS}^{29,30}$ Scanning tunneling microscopy has been used to experimentally verify the protection from backscattering in bismuth-based Tls. ${ }^{31-33}$ The spin-locking property in $\mathrm{Bi}_{2} \mathrm{Se}_{3}$ has been experimentally verified to some degree by measuring samples with metallic contacts, ${ }^{34}$ but there is much room for further study, especially in terms of how the thickness of a TI film relates to the relative contribution of the SS to the carrier dynamics. Films of about $6 \mathrm{QL}$ or thinner have exhibited hybridization of the Dirac cone ${ }^{35}$ introducing a gap to the SS. 
As-grown samples tend to be $n$-doped due to selenium (Se) vacancies, causing the Fermi level to be above the first set of SS, inside the CB. ${ }^{36}$ This causes the bulk to be conducting as well as the surfaces, making it more difficult to study ideal TI behavior. Efforts have been made to lower the Fermi level through doping or electrostatic gating. ${ }^{37-39}$ Fortunately, there exists a second set of surface states (SS2) ${ }^{40}$ about $1.7 \mathrm{eV}$ above the first set, giving an alternative for direct coupling of near-IR (infrared) photons to excite Fermi level electrons to SS2.

\section{4: Photocurrents}

\subsection{1: Light-matter interactions}

Many studies on particle dynamics in semiconductors and other novel materials take advantage of light-matter interactions. Hitting a sample with an ultrafast laser pulse provides a very quick (10-100 fs or even quicker in some cases), very intense stimulus to provoke non-equilibrium changes in the sample. This type of stimulus produces a wide range of phenomena that tell a lot about the symmetry of a lattice and electron interactions within a material.

Many of these phenomena may also be studied by hooking a sample up to metal contacts and applying a voltage. However, this has negative consequences that can be avoided by using the all-optical route. For example, there may be a build-up of space charge effects caused by band bending at a metalsemiconductor interface..$^{41,42}$ In these all-optical experiments there is no need for an interface of any type as the samples can be held in free space.

The many light-matter interactions available occur on various timescales. In order to completely understand a given material's light interaction, one must carefully deconstruct all contributions. Photocurrents in the context of this work are produced as a result of a plane electromagnetic wave described by

$$
\boldsymbol{E}(\boldsymbol{r}, t)=\boldsymbol{E}(\boldsymbol{q}, \omega) e^{-i \omega t+i \boldsymbol{q} r}+\boldsymbol{E}^{*}(\boldsymbol{q}, \omega) e^{i \omega t-i \boldsymbol{q} \boldsymbol{r}}
$$

where $\boldsymbol{E}(\boldsymbol{q}, \omega)$ is an electric field amplitude with wave vector $\boldsymbol{q}$ and frequency $\omega .{ }^{43}$ When an electric field is incident on matter, it forces positive and negative charges apart, creating a polarization $\boldsymbol{P}$. If the electric 
field is weak, there is a simple proportionality, but if it is strong, then $\boldsymbol{P}$ must be expanded into higher order terms:

$$
\boldsymbol{P}(\boldsymbol{k}, \omega)=\boldsymbol{P}^{(1)}(\boldsymbol{k}, \omega)+\boldsymbol{P}^{(2)}(\boldsymbol{k}, \omega)+\boldsymbol{P}^{(3)}(\boldsymbol{k}, \omega)+\cdots
$$

The first term is the simple weak-field term $\boldsymbol{P}^{(1)}(\boldsymbol{k}, \omega)=\chi^{(1)}(\boldsymbol{k}, \omega) \boldsymbol{E}(\boldsymbol{k}, \omega)$. The second term $\boldsymbol{P}^{(2)}(\boldsymbol{k}, \omega)=\chi^{(2)}\left(\boldsymbol{k}=\boldsymbol{k}_{i}+\boldsymbol{k}_{j}, \omega=\omega_{i}+\omega_{j}\right): \boldsymbol{E}\left(\boldsymbol{k}_{i}, \omega_{i}\right) \boldsymbol{E}\left(\boldsymbol{k}_{j}, \omega_{j}\right)$ generally follows the symmetry of the crystal lattice and vanishes under inversion symmetry. Examples of this $2^{\text {nd }}$-order nonlinear polarization are optical rectification $(\mathrm{OR})$ and shift currents (SCs), described in Section 1.4.2. The term $\boldsymbol{P}^{(3)}(\boldsymbol{k}, \omega)=$ $\chi^{(3)}\left(\boldsymbol{k}=\boldsymbol{k}_{i}+\boldsymbol{k}_{j}+\boldsymbol{k}_{l}, \omega=\omega_{i}+\omega_{j}+\omega_{l}\right): \boldsymbol{E}\left(\boldsymbol{k}_{i}, \omega_{i}\right) \boldsymbol{E}\left(\boldsymbol{k}_{j}, \omega_{j}\right) \boldsymbol{E}\left(\boldsymbol{k}_{l}, \omega_{l}\right)$ is nonvanishing for all materials, and its symmetry is typically isotropic. This $3^{\text {rd }}$-order polarization describes current injection, which is explored in Section 1.4.3. Following this pattern, all odd polarization terms $\boldsymbol{P}^{(1,3,5, \ldots)}(\boldsymbol{k}, \omega)$ are present in every material, but all even terms $\boldsymbol{P}^{(2,4,6, \ldots)}(\boldsymbol{k}, \omega)$ may vanish, depending on the crystal symmetry.

\subsection{2: Optical rectification and shift currents}

When an electric field $\boldsymbol{E}$ is incident on electrons, they experience the coulomb force $\boldsymbol{F}=q \boldsymbol{E}$. When this takes place in a material, the resulting current depends on how tightly bound the electrons are. The current $\boldsymbol{J}$ can be described by Ohm's Law:

$$
\boldsymbol{J}=\sigma \boldsymbol{E}
$$

Here, the ratio of the strength of the resulting current density produced by the given electric field is called the conductivity $\sigma$. If the photons involved are not energetic enough to excite electrons from their bound states to the $\mathrm{CB}$, the radiation will nonetheless provoke simple harmonic motion about the nuclei to which the electrons are localized. When the oscillation of the field direction is very fast (for example 800-nm light, which oscillates at a rate of $375 \mathrm{THz}$ ), then the polarization is partially described as the average position of the electron wavefunction. In a centrosymmetric system like that in Figure 1.7(a), the negatively charged electron wavefunction is centered on the positively charged nucleus, so the atom has no $2^{\text {nd }}$-order polarization, although it will always have an instantaneous polarization depending on where an electron is at any given moment. Even when radiation is incident as in Figure 1.7(b), electrons oscillate with the same 
strength in opposite directions, so although the electron cloud is forced to change shape the $2^{\text {nd }}$-order nonlinear polarization is still zero. Therefore, the polarization of the systems in both Figure $1.7(a)$ and (b) contains only odd terms $\boldsymbol{P}(\boldsymbol{k}, \omega)=\boldsymbol{P}^{(1)}(\boldsymbol{k}, \omega)+\boldsymbol{P}^{(3)}(\boldsymbol{k}, \omega)+\boldsymbol{P}^{(5)}(\boldsymbol{k}, \omega)+\cdots$.
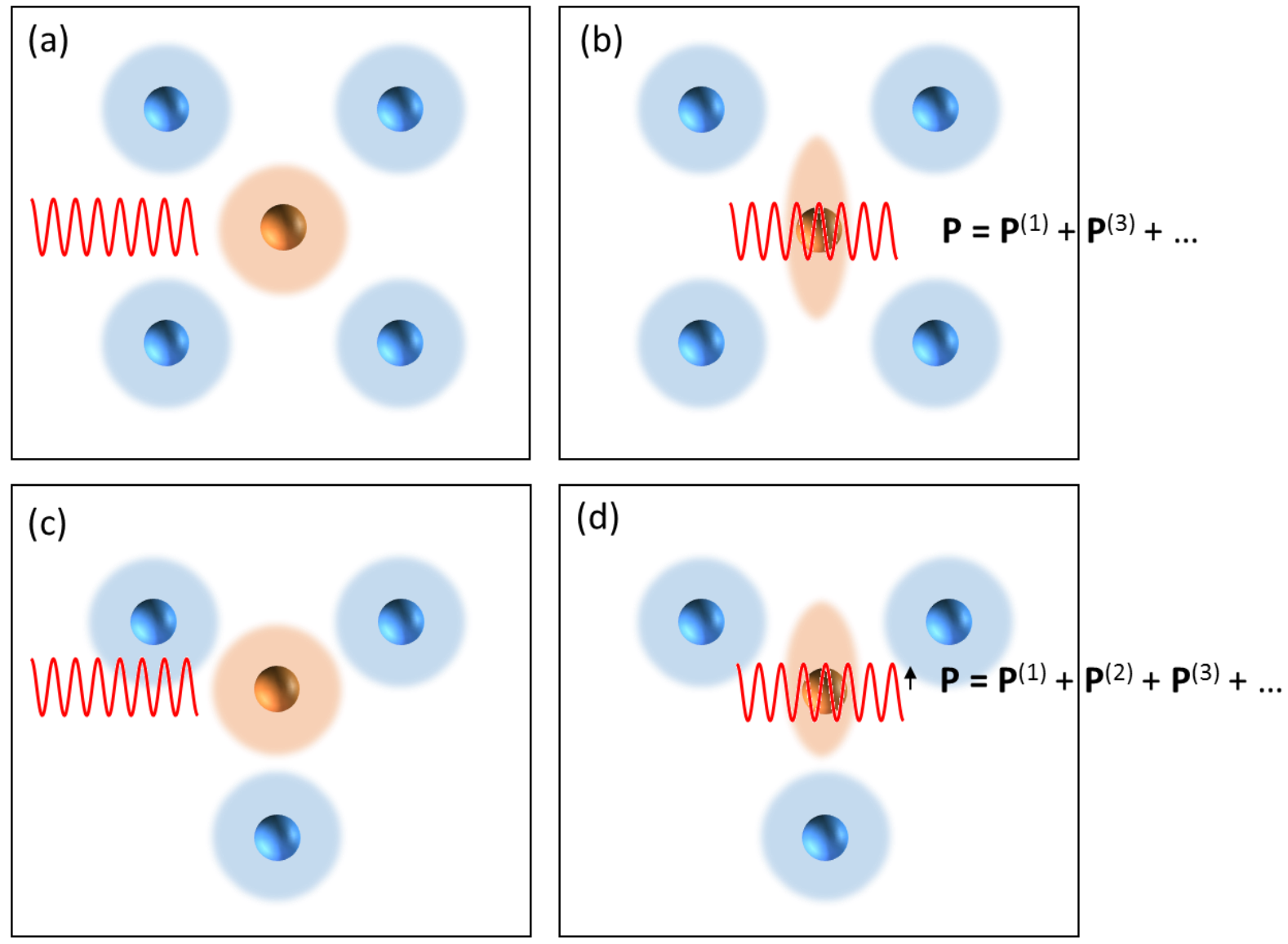

Figure 1.7: (a) A centrosymmetric crystal. (b) A quickly oscillating electric field alters the electron wave function symmetrically. (c) A non-centrosymmetric crystal. (d) An electric field stretches the electron wave function asymmetrically due to neighboring coulomb forces, resulting in the indicated polarization vector including the $2^{\text {nd }}$-order nonlinear polarization.

On the other hand, if a system does not have centrosymmetry, oscillating electrons will encounter a neighboring coulomb force in one direction that is weaker in the opposite direction. Figure 1.7(c) and (d) show a noncentrosymmetric system before and after photon stimulation. As the orange electron wavefunction oscillates, it experiences a repulsive force from the blue neighboring atom in one direction, while being free to move further in the opposite direction. The stronger the strength of the incident radiation, the more asymmetric the new wavefunction becomes, creating an average displacement of the negative charge from the positively charged nucleus. Since a quickly oscillating electric field creates a static 
nonlinear polarization, this process is known as rectification. In fact, in the case of Figure 1.7(d), all of the terms in Equation 1.5 are nonvanishing.

In the case of a pulsed laser used for the stimulation process, the amplitude of the oscillations gradually grows and then decreases within a gaussian envelope. Therefore, the OR signal follows the envelope of the pump pulse.

If the photon energy is enough to excite electrons across the material's band gap, a similar process occurs, but electron wavefunctions can then be re-localized from their original positions to neighboring atoms. This occurs due to a difference in the density of states (DOS), where the original atom may have more favorable VB states, but the neighboring atom has more favorable CB states. The result is an SC which can be described by

$$
J_{a}=\sigma^{a b b}(\omega) E_{b}(\omega) E_{b}(-\omega)
$$

In GaAs, OR and SC were observed and compared at 0.8 and $1.6 \mathrm{eV}$, respectively. ${ }^{44} \mathrm{SCs}$ occur when electrons promoted from the VB to the $\mathrm{CB}$ consequently have their wavefunctions re-localized from one atom to its nearest neighbor. ${ }^{45}$ This re-localization occurs in $\mathrm{Bi}_{2} \mathrm{Se}_{3}$ because the $\mathrm{DOS}$ is higher near the Se atoms in the VB, but the DOS is higher near the $\mathrm{Bi}$ atoms in the $\mathrm{CB},{ }^{12}$ so on average some electrons on promotion must shift from the Se atoms to nearest neighbor Bi atoms. In the bulk, each atom is a center of inversion symmetry, so electrons will shift isotropically on average. However, at the surface the inversion symmetry is broken, so this shift will produce an average current in a preferential direction. 


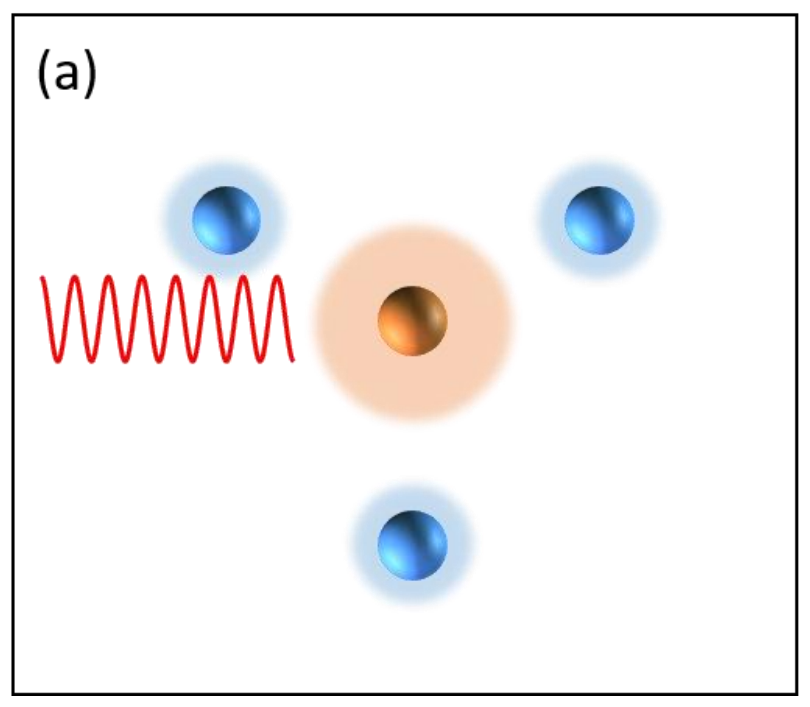

(b)

Figure 1.8: SC. (a) In its unexcited state, electrons are predominantly localized near the orange atom. After excitation, the wave function shifts along the polarization of the pump field, causing a redistribution of charge towards a blue atom.

It has recently been proposed ${ }^{46}$ that SC may be used as an alternative means of capturing solar energy in photovoltaic devices. Conventional Si solar cells can convert sunlight to electricity at a rate of about $400 \mathrm{~mA} / \mathrm{W}$. For the narrow-band gap materials proposed as SC photovoltaics, theory suggests that hundreds of $\mathrm{mA} / \mathrm{W}$ could be achieved, suggesting that their efficiency may soon outpace that of conventional solar cells.

\subsection{3: Current injection theory}

The third-order nonlinear optical process of current injection can be described by the equation

$$
\frac{d J_{i}^{i n j}}{d t}=2 \eta_{i j k l} E_{j}^{\omega} E_{k}^{\omega} E_{l}^{2 \omega} \sin \left(2 \phi_{\omega}-\phi_{2 \omega}\right)
$$

and the phase relationship between the two pump pulses is commonly written as $\Delta \phi=2 \phi_{\omega}-\phi_{2 \omega}$. A Fermi's Golden Rule calculation leads to the conclusion that the injection efficiency is proportional to the fourth-rank tensor $v^{(3)}(-2 \omega, \omega, \omega),{ }^{47}$ which is related to the sum of one- and two-photon excitation terms. ${ }^{48}$ For our particular geometry we can consider only the diagonal elements of the susceptibility tensor $\chi_{x x x x}^{(3)}{ }^{49}$ 


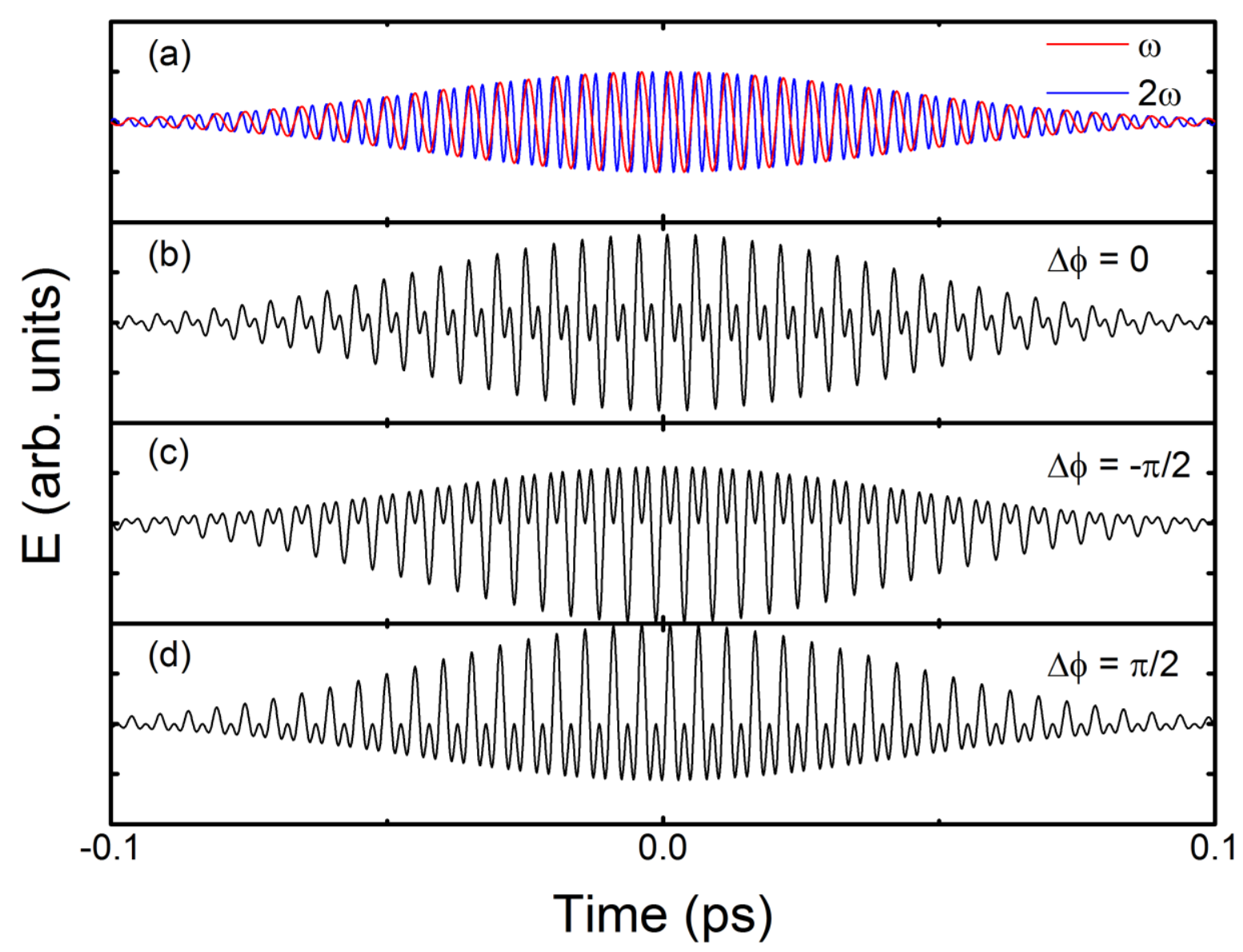

Figure 1.9: (a) Electric field for 100-fs laser pulses with center wavelengths of $1540 \mathrm{~nm}(\omega$, red) and $770 \mathrm{~nm}(2 \omega$, blue) with relative phase condition $\Delta \phi=0$. (b) The sum of the two pump electric fields in (a). (c) The sum of the pumps after shifting the $2 \omega$ pump by $-\lambda_{2 \omega} / 4$. (d) The sum of the pumps after shifting the $2 \omega$ pump by $\lambda_{2 \omega} / 4$.

The use of coherent control of ultrafast laser pulses to selectively excite charge carriers in semiconductors has allowed for significant advancement in the study and understanding of carrier dynamics in semiconductor materials. The application of two collinear pump pulses of frequencies $\omega$ and $2 \omega$ excites single-photon and two-photon interband absorption pathways and the relative phase $\Delta \phi$ between the pump pulses controls the interference pattern in crystal momentum space. ${ }^{48}$ This technique has been used with success to generate photocurrents in semiconductors such as silicon and $\mathrm{GaAs}{ }^{50,51}$ as well as graphene ${ }^{52}$ and carbon nanutubes. ${ }^{53}$ Above band-gap excitation of lower symmetry semiconductors, such as wurtzite crystals, results in electrons and holes being injected preferentially on one side of the Brillouin zone. ${ }^{54}$ Coherent control of photocurrents has the advantage of being non-contact and non-invasive, so currents 
can be produced and observed without the need for contact with metals which might significantly alter the properties of the material in question.

\subsection{4: Photon drag effect}

Photon drag is a second-order nonlinear phenomenon which occurs as a result of electron friction due to scattering. This effect requires a single linearly polarized obliquely incident laser beam, and describes the transfer of momentum from photons to electrons. Currents formed in this way can be described by the equation

$$
j_{\lambda}^{(d r a g)}=\Phi_{\lambda \mu \nu \eta}(\omega) \times \frac{i}{2}\left[E_{\nu}(\boldsymbol{r}, \omega) \frac{\partial E_{\eta}^{*}(\boldsymbol{r}, \omega)}{\partial x_{\mu}}-E_{\eta}^{*}(\boldsymbol{r}, \omega) \frac{\partial E_{v}(\boldsymbol{r}, \omega)}{\partial x_{\mu}}\right] .
$$

Photon drag currents have been observed in $p$-type germanium ${ }^{55}$ and graphene, ${ }^{56}$ as well as in three-dimensional TIs. ${ }^{57}$ Photon drag currents need to be kept in mind when considering the wealth of effects that occur simultaneously in any material. However, because photon drag currents are expected to be parallel to the $k$-vector of the pump beam, they are undetectable by the methods used in Chapters 4 and 5 below.

\subsection{5: Photocurrents in topological insulators}

Photocurrents are a versatile tool for studying excited-state charge carrier dynamics in atoms, molecules, and solids. Even for the simplest semiconductors, subject to excitation in the limit of long pump pulses at a single carrier frequency, there is a complex assortment of photocurrents. "One-color effects" arise in noncentrosymmetric crystals, such as GaAs. If the photon energy $\hbar \omega$ is below the band gap $E_{g}$, the pump pulse can induce OR; here in a simple description the induced dipole moment per unit volume follows the intensity of the pulse. If $\hbar \omega>E_{g}$, then SC can arise, where in a simple description the current density follows the intensity of the pulse, as the center of charge within a unit cell moves as light is absorbed and electrons are promoted from VB to CB. ${ }^{44,45}$ Finally, for semiconductor crystals with a lower symmetry than GaAs, such as those that form in the wurtzite structure, excitation above the band gap can also lead to an IC, where electrons and holes are injected preferentially on one side of the Brillouin zone; here in a simple description, neglecting scattering, the time rate of change of the injected current follows the intensity. These 
three processes of OR, SC, and IC are all aspects of the same optical response, and the connections between them have been studied in detail for the noncentrosymmetric crystals in which they arise. ${ }^{58}$ Typically the currents due to injection are the largest if they survive, and if not the SC are larger than the OR response, but there are exceptions: Bieler et al. ${ }^{59}$ found that SC are larger than IC in (110)-grown GaAs/AIGaAs quantum wells, which have reduced symmetry due to quantum confinement.

In centrosymmetric crystals, such as silicon, one-color effects do not survive. However, photocurrents can arise from the interference of light with carrier frequencies at $\omega$ and $2 \omega$ when $2 \hbar \omega$ crosses the gap..$^{50}$ This principle was first described in molecules by Brumer and Shapiro using $\omega$ and $3 \omega$ continuous wave (CW) lasers. ${ }^{60}$ "Two-color" injection currents (ICs) survive in any material, as do two-color SC and OR currents. In fact, two-color ICs were first studied in GaAs crystals. ${ }^{51}$ The IC is expected to dominate when $2 \hbar \omega$ crosses the band gap.

Graphene has a band structure that distinguishes it from the usual semiconductors, in that its states near the six symmetric $K$ points form a Dirac cone with massless dispersion. Two-color photocurrents have been observed, ${ }^{52}$ and the expressions for the nonlinear optical response that describes them have been worked out in detail 61 for frequencies where states near the $K$ points are important, with scattering included phenomenologically. Unlike most semiconductors, where two-color ICs have been studied for $\hbar \omega<E_{g}<$ $2 \hbar \omega$, in undoped graphene one-photon absorption (1PA) is possible at both $2 \omega$ and $\omega$, and 1PA of the fundamental interferes with the current injection process. ${ }^{62}$ Yet since the lattice structure has inversion symmetry there are no one-color effects.

The $\mathrm{TI} \mathrm{Bi}_{2} \mathrm{Se}_{3}$ has a richer band structure, containing both a direct band gap in the bulk and graphene-like massless conducting states near the surface. It has been proposed ${ }^{63}$ that two-color ICs can be injected by a laser pump and its second harmonic, and this was observed ${ }^{64}$ in $\mathrm{Bi}_{2} \mathrm{Se}_{3}$ thin films using a terahertz $(\mathrm{THz})$ probe.

Many surface effects come into play at interfaces between Tls and other materials, some of which can lead to exotic phenomena, ${ }^{65,66}$ but very often they only introduce complications in experiments designed to study the topological SS. ${ }^{67}$ This suggests that photocurrents would constitute an ideal probe, since 
contacts at the surface can be avoided. Due to the 0.3-eV band gap of $\mathrm{Bi}_{2} \mathrm{Se}_{3}$, optoelectronic applications involving explicitly the properties of the topological SS had been thought to require optical fields with midto-far IR frequencies. However, the discovery of SS2 shows that surface-to-surface optical transitions can be excited using near-IR commercial solid-state lasers $(\sim 0.8 \mathrm{eV})$ that offer the possibility of extreme control of dynamics.

Just as important for the study of a $\mathrm{TI}$ such as $\mathrm{Bi}_{2} \mathrm{Se}_{3}$ is that in the bulk the material is centrosymmetric, and thus no one-color effects can arise. ${ }^{14,20,68}$ Nonetheless, if it is excited with light at $\omega$ and $2 \omega$, with $2 \hbar \omega>E_{g}$, two-color ICs will appear. At the surface the symmetry is broken, and in fact a onecolor SC is allowed. ${ }^{41,42,68}$ The presence of both two-color and one-color photocurrents is both a challenge and an opportunity. The challenge is to be able to separate the two effects and identify just to what an experiment is sensitive. The opportunity is that by examining the interplay between these two photocurrents a noninvasive study of the electronic states both in the bulk and at the surface should be possible. In Chapter 5, we demonstrate a scheme for detection of these two types of photocurrents. For each photocurrent the dependence on the angle between the light polarization and the crystal lattice axis is measured and found to agree with theoretical predictions. We present a comparison of the strengths of the SCs and ICs, and outline a method for isolating the IC using simple measurements of the total two-color photocurrent.

\section{5: Summary of chapters}

Chapter 2 will discuss the variety of experiments available utilizing and relating to $\mathrm{THz}$ radiation. Fundamentals of $\mathrm{THz}$ detection will be discussed including the difficulties and characteristics. Methods of THz generation will be discussed including OR and photocurrent generation via SC and IC, and a variety of samples are compared in terms of their viability as $\mathrm{THz}$ emitters. Then, the collection and analysis of time-resolved $\mathrm{THz}$ measurements will be explained in terms of characteristics and limitations of the methods used. Additionally, the conductivity of free carriers in solar harvesting materials will be probed by transmission of $\mathrm{THz}$ radiation, which will be related to how adding a dopant affects the band structure. 
In Chapter 3, temperature dependent THz time domain spectroscopy (THz-TDS) is used to analyze magnon resonances in $\mathrm{AF} \mathrm{MnF}_{2}$. The resonance frequency of the magnon is examined as a function of temperature as the material transitions from its AF phase to the paramagnetic. This temperature- and frequency-dependence is then compared to theoretical models and used to determine the degree of coupling between nearest neighbor spins. The $\mathrm{THz}$ refractive index is also measured as a function of temperature and the phenomena which dictate its behavior are identified and modeled.

In Chapter 4, photocurrents are optically injected into molecular beam epitaxy-grown $\mathrm{Bi}_{2} \mathrm{Se}_{3} .{ }^{64} \mathrm{The}$ photocurrents originate from the well-known nonlinear all-optical processes IC and SC, and their behavior corresponds to that in simpler semiconductor samples from the literature. In addition to the proof-of-principle demonstration we also discuss the outlook including further parameter space that will be explored in future studies.

In Chapter 5 we deal with the problem of isolating multiple contributions to the photocurrent signal. The simultaneous occurrence of multiple sources of currents is unavoidable so a method is developed for the isolation of the two contributions. By using an in-depth analysis, we are able to compare IC and SC magnitudes independently across a variety of conditions and samples.

Chapter 6 will deal with the fundamental light absorption properties of $\mathrm{Bi}_{2} \mathrm{Se}_{3}$ as measured by various methods. 1PA coefficients are recorded and compared with the band structure of the material, and z-scan and non-degenerate transient absorption measurements are used to explore the nonlinear absorption properties. These fundamental constants are then used to explain the behavior of IC experiments. 


\section{Chapter 2: Terahertz Experiments}

$\mathrm{THz}$ radiation falls within somewhat of a technology gap between microwave and IR wavelengths. The exact range of wavelengths that belongs in the $\mathrm{THz}$ category is up for debate, but the definition most often refers to frequencies $v=0.1-10 \mathrm{THz}$, which corresponds to wavelengths $\lambda=3-0.03 \mathrm{~mm}$, or photon energies $E=0.4-40 \mathrm{meV}$. Because of this, THz waves are sometimes also referred to as millimeter waves or submillimeter waves.

The reason the $\mathrm{THz}$ portion of the spectrum is sometimes referred to as a "gap" is because lower energy radio and microwave radiation is often used in electronic technologies, and higher energy IR and ultraviolet radiation is used in well-established optical technologies. $\mathrm{THz}$ bridges a gap between the two wherein it shares some properties of each side, but until recently has not seen serious research and development of sources, detectors, and waveguides. THz shows tremendous potential in a wide variety of areas including imaging and remote sensing, quality control, and materials characterization, but much work needs to be done in order to develop commercial THz products.

One of the advantages of all-optical and $\mathrm{THz}$ experiments is the degree of control experimentally that can be exploited to explore parameter space. The polarization of a laser pulse with respect to a given sample axis can be easily controlled using waveplates or even by simply rotating the sample about the optical axis, which translates to a modification of the direction of the force of the stimulus. The photon energy can also be easily modified given the right equipment. In this work an optical parametric amplifier (OPA) is used, in which difference frequency generation is used to generate a near-IR wavelength between 1.1 and $2.5 \mu \mathrm{m}$. This allows for very accurate access to specific energy states in a system. For example, by lowering the photon energy to below the band gap of a material, certain interactions that require promotion of electrons to the $\mathrm{CB}$ are turned off, allowing the consequences of those interactions to be directly observed. Of course, the strength of the stimulus can also be easily modified using a set of calibrated neutral density (ND) filters. In many cases, pumping very weakly has a linear and uninteresting effect on the electrons in a system, but increasing the power provided tends to push the signal into a nonlinear regime where a rich wealth of interesting physics can be explored. 


\section{1: Terahertz generation}

This research is focused specifically on the use of THz pulses in spectroscopy. Single-cycle broadband pulses are generated using OR in a chalcopyrite generation crystal: For this work, the generation crystal was either 0.5-mm thick, (110)-cut zinc germanium diphosphide ( $Z n G e P_{2}$ or ZGP);69 or 0.5-mm thick, (110)-cut cadmium silicon diphosphide $\left(\mathrm{CdSiP}_{2}\right.$ or $\left.\mathrm{CSP}\right)$. To accomplish this, the generation crystal is pumped with a 100-fs pulse with a carrier wavelength of either $800 \mathrm{~nm}$, or an OPA wavelength $1.1-2.5 \mu \mathrm{m}$. Electrons vibrate at the carrier frequency, but due to asymmetry in the crystal lattice along the pump polarization vector, their average wavefunctions become spatially offset, creating a nonlinear polarization which follows the pump envelope as described in Section 1.4.2.

The chalcopyrites ZGP, CSP, and cadmium germanium diphosphide (CdGeP 2 or CGP) are all type II-IV-V $V_{2}$ compounds which have a similar structure and therefore should behave similarly under optical stimulation. ${ }^{70}$ All three crystals belong to the tetragonal point group $\overline{4} 2 m$, and crystallize in the space group $D_{2 d}^{21} \cdot{ }^{71}$ Their chalcopyrite structure is closely related to zinc-blende and wurtzite crystal structures. Their known nonlinear optical properties are listed in Table 1. In order to fully model the power dependence of $\mathrm{THz}$ generation in the materials, the rest of the table would need to be completed.

\begin{tabular}{|l|l|l|l|}
\hline & $\mathrm{d}_{36}(\mathrm{pm} / \mathrm{V})$ & $\alpha\left(\mathrm{cm}^{-1}\right)$ & $\gamma\left(\mathrm{cm}^{3} / \mathrm{GW}^{2}\right)$ \\
\hline CGP & $162^{72}$ & $3.5^{72}$ & \\
\hline CSP & $92^{73}$ & $0.1^{74}$ & \\
\hline ZGP & $75^{75}$ & $1.8^{74,75}$ & $0.09^{75}$ \\
\hline
\end{tabular}

Table 1: Nonlinear tensor element $d_{36}$, 1PA coefficient $\alpha$, and three-photon absorption (3PA) coefficient $\gamma$ of CGP, CSP, and ZGP at $1300 \mathrm{~nm}$.

Figure 2.1 shows the setup used to generate $\mathrm{THz}$ in a chalcopyrite sample. The gate pulse is a 100-fs, 800-nm laser pulse derived from a regenerative Ti:sapphire laser amplifier with a $1 \mathrm{kHz}$ repetition rate. $50 \%$ of this pulse is split off and sent through an OPA, which allows color tuning in the near-IR range from about 0.5 to $1.0 \mathrm{eV}$. The color-tuned pulse becomes the trigger pulse, which is then normally incident on one of the chalcopyrite samples. OR in the sample then produces $\mathrm{THz}$ radiation which radiates outward in a cone. This radiation is then collected using off-axis parabolic mirrors, which refocus the radiation on a 0.3-mm thick ZnTe crystal, which is used as an electro-optic (EO) sampling crystal. The EO crystal 
experiences a birefringence that is proportional to the $\mathrm{THz}$ field, and the gate pulse experiences the birefringence as it passes through the EO crystal at a time delay $t$.

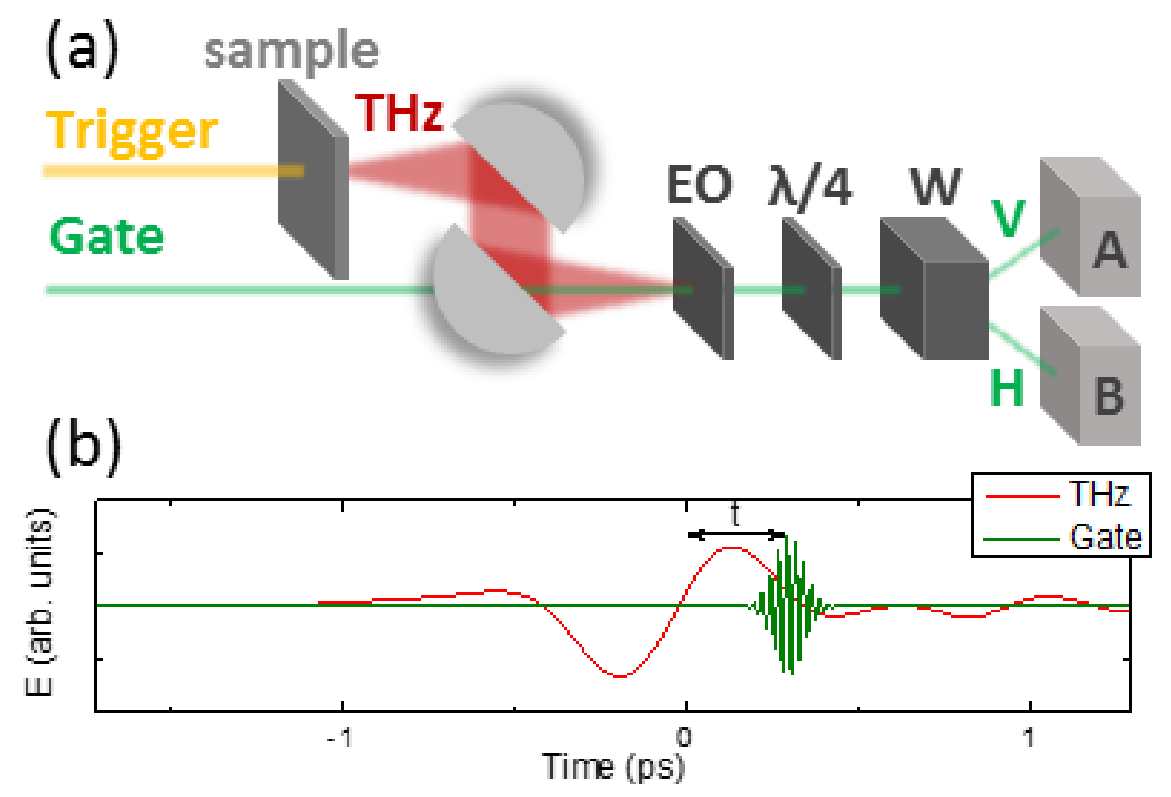

Figure 2.1: Schematic of $\mathrm{THz}$ generation experiment. (a) $\mathrm{EO}=$ electro-optic crystal; $N / 4=$ quarter-wave plate; $\mathrm{W}=$ Wollaston prism; $\mathrm{V}=$ vertically polarized pulse; $\mathrm{H}=$ horizontally polarized pulse; $\mathrm{A}$ and $\mathrm{B}=$ balanced photodiodes. (b) $\mathrm{THz}$ and gate pulses overlapped with a time delay $t$ in the EO sampling crystal.

Without the presence of $\mathrm{THz}$, the gate pulse is converted from linear polarization to circular in the quarter-wave plate. The Wollaston prism then redirects the orthogonally polarized components to photodiodes. Since both components of the gate are the same intensity, the signal detected is $A-B=0$. When $\mathrm{THz}$ is present, the birefringence in the EO crystal causes the difference between the two components to change, and the signal detected $A-B$ is proportional to the $\mathrm{THz}$ electric field.

The graph in Figure 2.2 shows the dependence of the amplitude of the $\mathrm{THz}$ electric field $\left|E_{T H z}\right|$ on the intensity $I_{0}$ of the pump pulse. In the case of all three chalcopyrites, at low intensity the field strength increases linearly. However, at higher pump intensities the pump beam is attenuated as it passes through the crystal according to Beer's law: $d I(z) / d z=-\alpha I(z)-\beta I^{2}(z)-\gamma I^{3}(z)+\cdots$, where $\alpha, \beta$, and $\gamma$ are the 1PA, 2PA, and 3PA coefficients, respectively. This results in the sublinear behavior which can be approximated with the simple model $\left|E_{T H z}\right|=A\left(I_{0}\right)^{B}$ with $B \approx 0.5$ in the case of all three samples. 


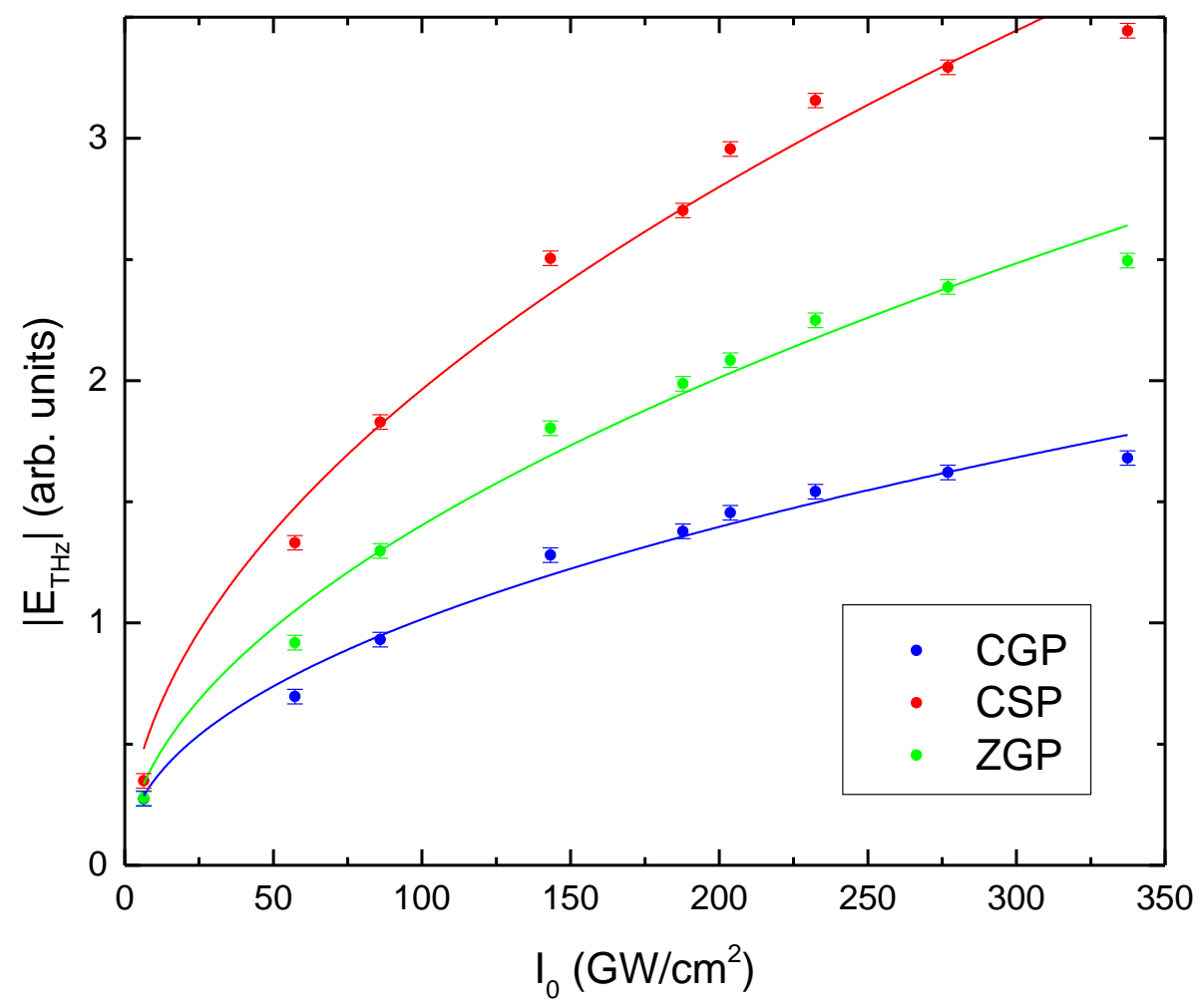

Figure 2.2: Power dependence of $\mathrm{THz}$ signal produced by chalcopyrites using a $1300-\mathrm{nm}$ pump pulse. Solid curves are fits of the form $\left|E_{T H z}\right|=A\left(I_{0}\right)^{B}$. Error bars were approximated from the signal to noise ratio of a single $\mathrm{THz}$ transient.

The wavelength dependence shown in Figure 2.3 shows a similar behavior for all three materials. The pump intensity was set to $100 \mathrm{GW} / \mathrm{cm}^{2}$ to ensure operation in the linear power regime. The photon energy of the pump was then varied via the OPA in the near-IR range from about 0.5 to about $1.0 \mathrm{eV}$. THz transients $|E(t)|$ were then recorded, and the relative field strength was calculated using $\left|E_{T H z}\right|=\sqrt{\int E^{2} d t}$ over the duration of the main pulse to mitigate the noise. Above about $0.6 \mathrm{eV}, \mathrm{CSP}$ is consistently the most efficient material for generating $\mathrm{THz}$, but below $0.6 \mathrm{eV}$ it drops off significantly compared to CGP and ZGP. For those lower photon energies, CGP appears to be the most efficient of the three materials. However, they are also within the same order of magnitude, so all three may be used interchangeably for future $\mathrm{THz}$ generation if the maximum achievable field amplitude is not crucial to the success of the experiment. 


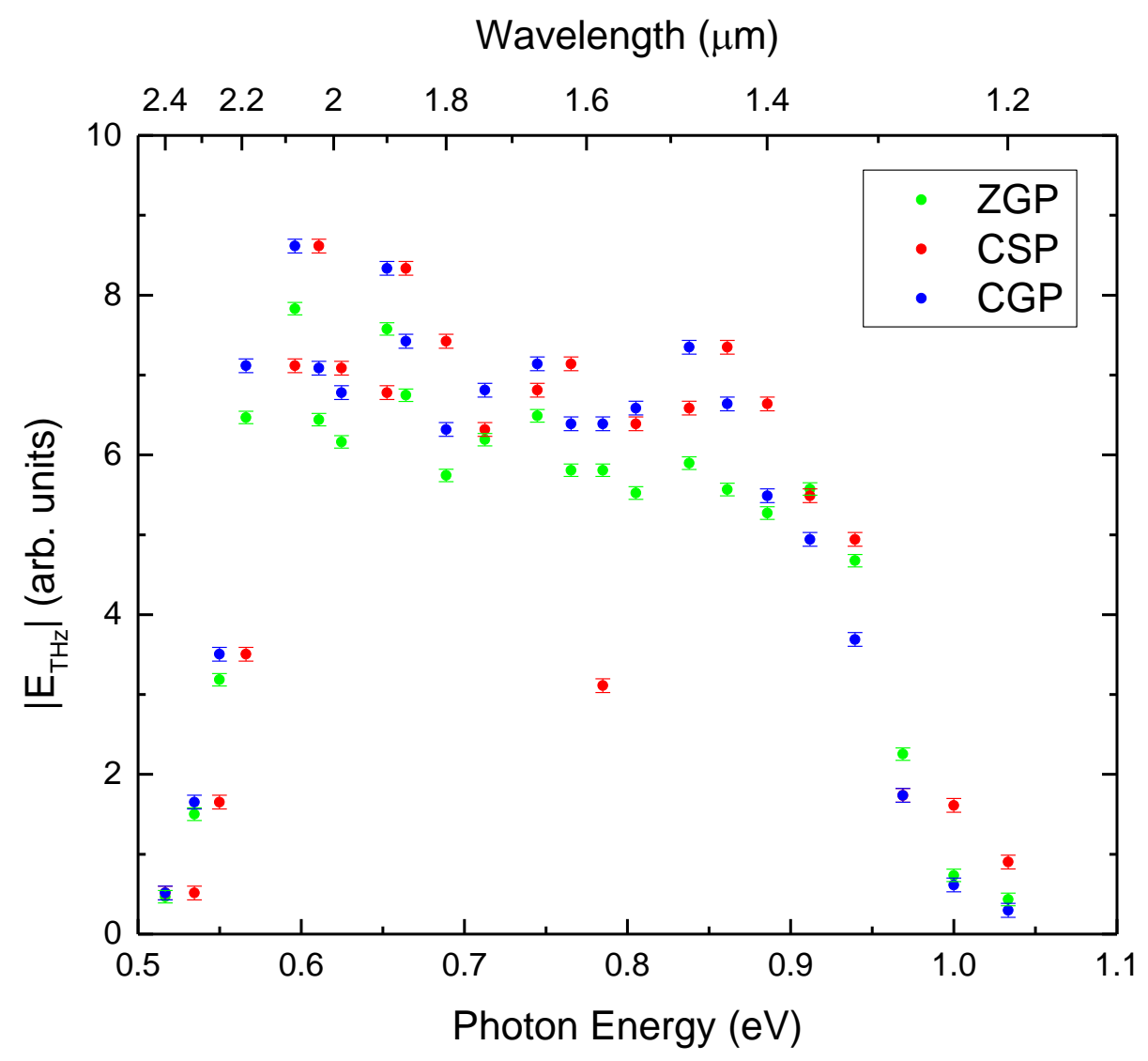

Figure 2.3: Pump photon energy dependence of $\mathrm{THz}$ signal produced by chalcopyrites using a pulse in the linear power regime. Error bars are estimated based on the signal to noise ratio of a single transient.

In summary, it has been shown that the chalcopyrites CGP and CSP are efficient alternatives to ZGP for THz generation through OR. The three materials studied here show similar behavior in both their power and wavelength dependences. All three can be used for $\mathrm{THz}$ sources in the optical experiments performed in Chapter 3.

\section{2: Terahertz detection}

A linearly polarized THz pulse $E_{x}(\omega)$ emitted by a sample is related to the EO signal $S_{x}(\omega)$ that we detect through a relation involving the transfer function $h(\omega)$,

$$
S_{x}(\omega)=h(\omega) E_{x}(\omega) .
$$


The transfer function describes attenuation and retardation on the $\mathrm{THz}$ pulse as a function of frequency $\omega$. There are several factors involved in the modification of the pulse, potentially including spatial truncation from the finite size of the reflective optics, attenuation effects caused by atmosphere, and the limited bandwidth sensitivity of the detection crystal. The transfer function can be experimentally determined, but because the exact shape of the $\mathrm{THz}$ waveform generally exceeds the scope of this work, we assume $S_{x}(\omega) \propto E_{x}(\omega)$ is a valid approximation.

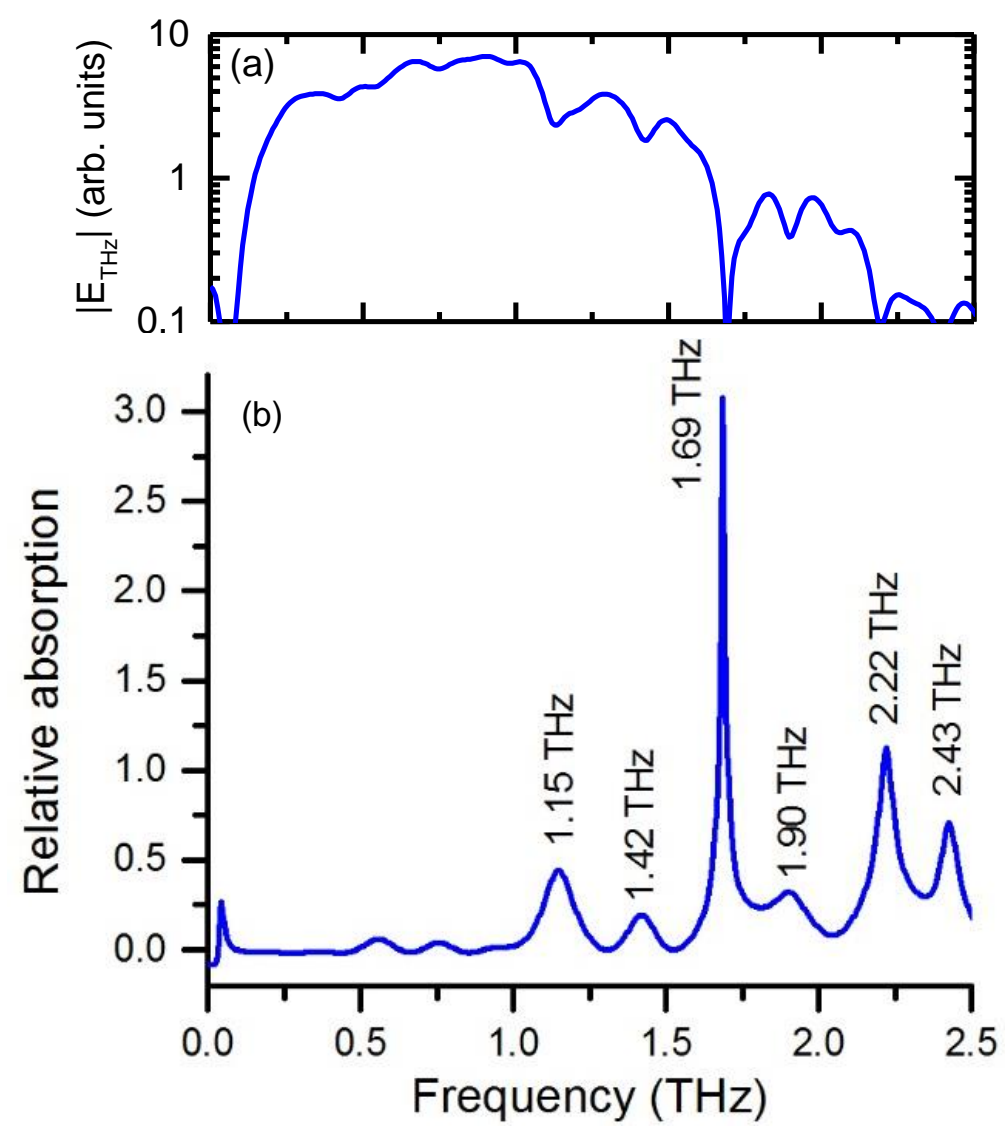

Figure 2.4: Atmospheric absorption of $\mathrm{THz}$ radiation. (a) Reference pulse traveling through atmosphere. (b) Relative absorption $A$ of atmosphere compared to $\mathrm{N}_{2}$ purge. Frequency labels are peaks caused by water absorption lines.

Propagation through atmosphere is mostly affected by the humidity present. $\mathrm{THz}$ is strongly absorbed by atmospheric water vapor at several frequencies, including 1.16, 1.41, 1.67, 1.92, 2.26, and 2.39 $\mathrm{THz} .{ }^{76,77}$ These absorption lines create problems with spectroscopy experiments because the weakened signal at these frequencies causes a higher signal-to-noise ratio. In order to alleviate this to some degree, an enclosed chamber envelops the entire path of the $\mathrm{THz}$ radiation for experiments in which 
this is a problematic issue, and nitrogen $\left(\mathrm{N}_{2}\right)$ is slowly and continuously pumped into the chamber, which has the effect of removing water vapor from the chamber and increasing the transmitted signal at the absorption line frequencies. Figure 2.4 shows the relative absorption in atmosphere compared to the purge chamber, calculated as $A=-\ln \left(I_{\text {nopurge }} / I_{\text {purge }}\right)$, demonstrating that the $\mathrm{N}_{2}$ purge dramatically increases the signal at several frequencies within our range of interest. Therefore, we know that the transfer function for an unpurged experiment must have the form $h(\omega)=h_{1}(\omega) e^{-A}$, in which $h_{1}(\omega)$ represents the remainder of the unaccounted-for contributions, including the reflective optics and the detection crystal.

Most of the experiments carried out in this work do not use a purge chamber. The absorption lines do not obscure the magnon features studied in the $\mathrm{MnF}_{2}$, and spectroscopy has not yet become a priority in the study of $\mathrm{Bi}_{2} \mathrm{Se}_{3}$.

\section{3: Terahertz transmission}

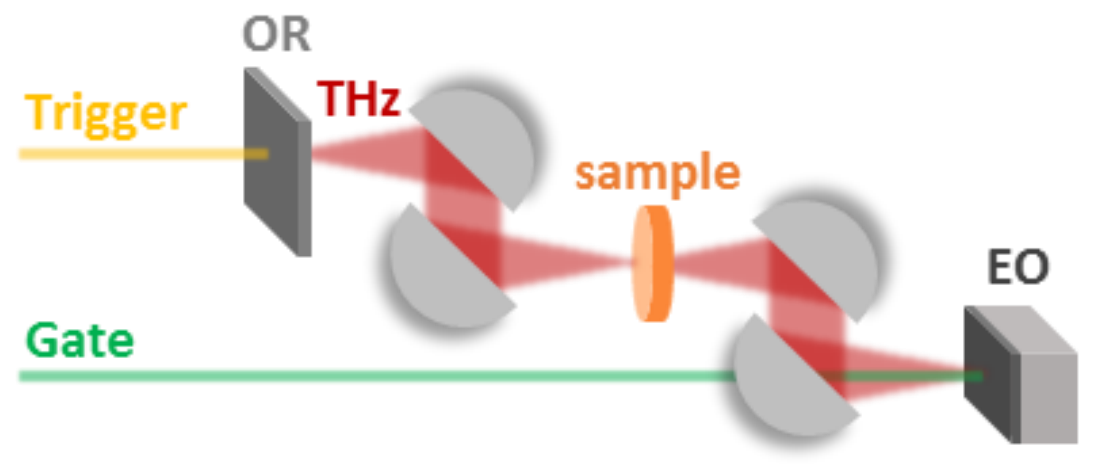

Figure 2.5: Schematic of $\mathrm{THz}$ transmission experiments. $\mathrm{OR}=$ Optical rectification crystal for $\mathrm{THz}$ generation; EO = electro-optic detection scheme, shown in detail in Figure 2.1.

In the following sections, $\mathrm{THz}$ radiation is transmitted through a sample. To accomplish this, a second set of parabolic mirrors are implemented so the $\mathrm{THz}$ can be passed through an intermediate focus where the sample is inserted, as in Figure 2.5.

\subsection{1: Conductivity}

When $\mathrm{THz}$ radiation passes through a material, its electric field can interact with the charged particles in the material. If the interaction cross-section is negligible, no energy is lost to the material, so no 
current is produced, and the conductivity $\sigma=0$ in Equation 1.6. However, in a metal, there are many free electrons that can be moved easily by the incident field, and $\sigma \gg 0$. In this case, energy from the radiation is lost to heating from the motion of electrons. This energy loss manifests itself in an attenuation of the radiation which can be detected on the output side of the material. Therefore, by comparing the output radiation to the amount sent into the material, one can directly obtain the value of the conductivity.

Additionally, by passing a broadband pulse of radiation through the material and measuring the output spectrum, the experiment becomes frequency resolved, allowing an observation of any resonances the electrons in the material might experience.

Furthermore, if a time-resolved experiment is used, one can detect not only the change in amplitude of the radiation, but the change in phase as well. And in this manner the entire complex conductivity can be resolved over a broad frequency range can be recorded using a $\mathrm{THz}$ conductivity experiment.

The complex optical conductivity $\sigma(\omega)$ of a material contains both the real and imaginary parts of the refractive index. The relationship between refractive index and conductivity is

$$
\begin{gathered}
\sigma_{1}=\omega \varepsilon_{0}\left(2 n \kappa-\varepsilon_{L, 2}\right), \\
\sigma_{2}=\omega \varepsilon_{0}\left(\varepsilon_{L, 1}-n^{2}+\kappa^{2}\right),
\end{gathered}
$$

where $\omega$ is the frequency of the optical field, $\varepsilon_{0}$ is the permittivity of free space, $n$ and $\kappa$ are the real and imaginary parts of the refractive index, and $\varepsilon_{L, 1}$ and $\varepsilon_{L, 2}$ are the real and imaginary parts of the lattice component of the permittivity (constants). THz is generated by OR in ZGP, ${ }^{78}$ and then refocused and transmitted through the sample of interest.

When a $\mathrm{THz}$ pulse is transmitted through a sample without the presence of a probe pulse, the complex transmission is given by

$$
T(\omega)=|T(\omega)| e^{i \phi(\omega)}=E_{s}(\omega) / E_{r}(\omega)
$$


where $E_{s}(\omega)$ is the complex $\mathrm{THz}$ electric field after having passed through the sample, and $E_{r}(\omega)$ is the reference field with no sample in the path. The conductivity can then be recovered from the transmission using the equation

$$
T(\omega)=\frac{2}{2+i \sigma d Z_{0}}
$$

where $d$ is the sample thickness and $Z_{0}$ is the impedance of free space.

\subsection{2: Conductivity in lanthanum dititanate}

The usefulness of $\mathrm{THz}$ conductivity experiments is demonstrated on titanium dioxide $\left(\mathrm{TiO}_{2}\right)$. Solar water splitting is a promising goal in the field of photovoltaics, for which $\mathrm{TiO}_{2}$ is a solid candidate. ${ }^{79}$ Nitrogen doping of $\mathrm{TiO}_{2}$ is suggested as a way to improve the active bandwidth of the material and hence the percentage of the solar spectrum which can be utilized. Here, the dopant states produced by nitrogen were investigated by measuring the static $\mathrm{THz}$ conductivity.

Doping a semiconductor can extend the light absorption range, however, it usually introduces the mid-gap states, reducing the charge carrier lifetime. A report ${ }^{79}$ shows that doping lanthanum dititanate $\left(\mathrm{La}_{2} \mathrm{Ti}_{2} \mathrm{O}_{7}\right.$ or LTO) with nitrogen (NLTO) extends the VB edge by creating a continuum of dopant states, increasing the light absorption edge from $380 \mathrm{~nm}$ to $550 \mathrm{~nm}$ without adding mid-gap states. The dopant states are experimentally resolved in the excited state by correlating transient absorption spectroscopy with a supercontinuum probe and density functional theory prediction. The lack of mid-gap states is further confirmed by measuring the excited state lifetimes, which reveal that the shifted band edge only increased carrier thermalization rates to the band edge and not interband charge recombination under both ultraviolet and visible excitation. 

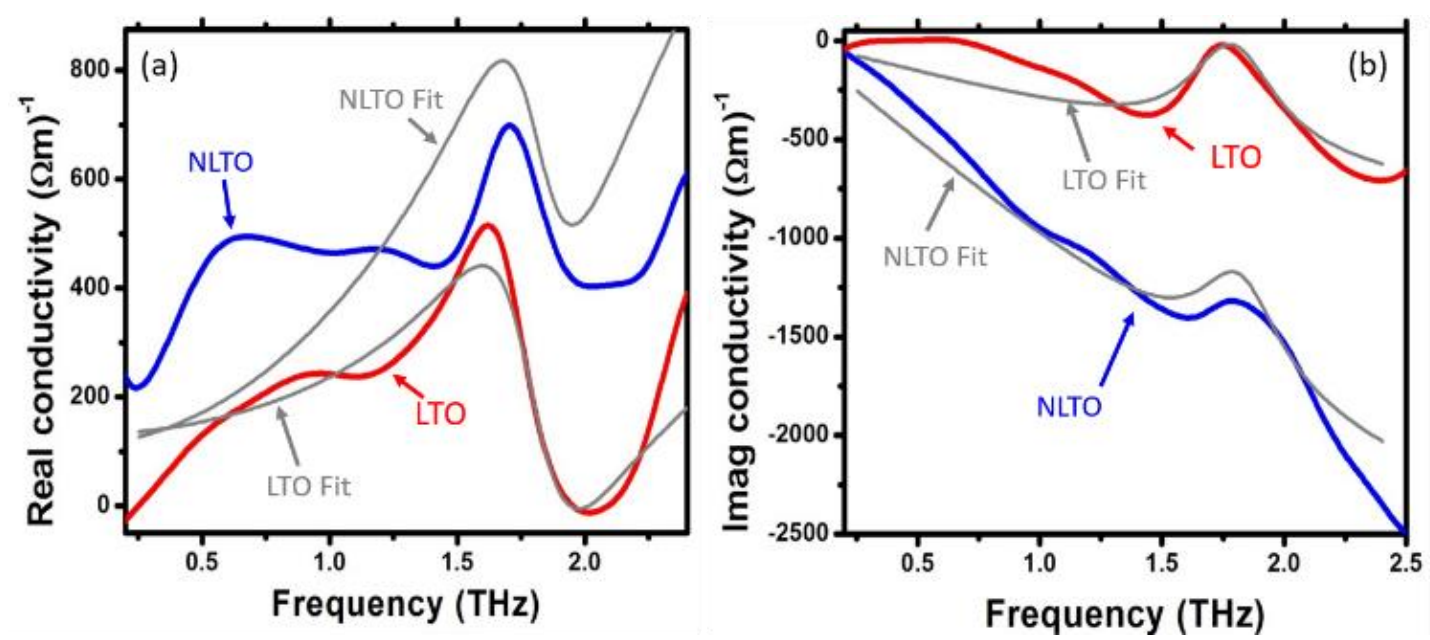

Figure 2.6: Conductivity measured through THz spectroscopy. LTO (red) and NLTO (blue) with (a) real and (b) imaginary conductivity. The nitrogen dopant increased the imaginary component of conductivity, corresponding to loss, but the frequency dependence stayed nearly identical. The grey lines show the fitting to a combined Drude-Smith and Lorentzian model.

The density functional theory prediction was further investigated by $\mathrm{THz}$ spectroscopy (Figure 2.6).

The $\mathrm{THz}$ light has a wavelength near the microwave region, allowing the frequency-dependent absorption to be related to the conductivity. ${ }^{80}$ The negative frequency dependence of the imaginary part of the conductivity suggests a Drude-Smith behavior in LTO, in which case conduction was limited by transport at interfaces, consistent with the nanoparticle morphology. ${ }^{81} \mathrm{~A}$ resonance was also seen at $1.8 \mathrm{THz}$ which corresponded to polar phonons. ${ }^{80}$ When doped, a noticeable change in the magnitude of both real and imaginary conductivity occurred, but the frequency dependence remained unchanged, as observed in the few-parameter Drude-Smith plus Lorentzian model, grey lines in Figure 2.6. ${ }^{81}$ The model indicated that the conduction mechanism was identical, but with a three times decrease in the zero-frequency conductivity in agreement with the DFT band structure. ${ }^{82}$ If the dopant states were not hybridized to form a continuum, excited carriers would have been trapped and conduction would occur by hopping, thus changing the frequency dependence in Figure 2.6.80

Doping LTO with nitrogen extended the light absorption range without reducing the charge carrier lifetime and mobility. This was only possible because the dopant created a continuum of states above the VB that acted to effectively shift the band edge of the material. The shifted band edge enhanced the light absorption while maintaining the carrier lifetime by avoiding new charge recombination routes from the mid- 
gap trap states commonly found in doped materials. This type of dopant strategy can enhance the visiblelight photocatalytic activity of semiconductors without jeopardizing the UV performance.

\section{4: Experimental considerations}

In the creation, collection, and transmission of $\mathrm{THz}$ radiation there are several factors that lead to distinct changes in the shape of the measured transients. In an attempt to understand the effects that the detection configuration has on the shape of the detected transient, various detection parameters were individually varied to show the relative effect of each. In a complete picture, every parameter needs to be simultaneously optimized to obtain the correct signal.

In the following experiments the $\mathrm{THz}$ radiation resulting from a single $1540-\mathrm{nm}$ pump pulse on 35- $\mathrm{QL} \mathrm{Bi}_{2} \mathrm{Se}_{3}$ was recorded as a function of several detection parameters. The $\mathrm{THz}$ was generated and detected as in Figure 2.1, where the sample is $\mathrm{Bi}_{2} \mathrm{Se}_{3}$, except with two pairs of parabolic mirrors used as in Figure 2.5. For detection, time $=0$ was arbitrarily chosen to be a point before the onset of the $\mathrm{THz}$ pulse. For adjustment of the last parabolic mirror downstream, an angle control knob was turned in increments of a quarter-turn, and geometry was used to convert this increment to a linear displacement on the $\mathrm{ZnTe}$ crystal. A similar method was used to adjust the gate pulse, with increments of $1 / 16$-turn used on the last available gate mirror.

In Figure 2.7, Figure 2.8, Figure 2.9, Figure 2.10, and Figure 2.11, the inset in (a) shows the unaltered $\mathrm{THz}$ traces detected by the lock-in amplifier. The main part (a) illustrates the shape change by normalizing and time shifting to line up the positive maximum of each transient. In (b), the signal "optimization" is shown by the blue trend, which is the strength of the positive maximum. Integrating the pulse over the entire time range gives a similar trend to the blue, with the peak at the same location. In (b), the red is the absolute strength of the first (negative) peak divided by the second (positive) peak. Therefore, this is a rough method of tracking the shape change as a function of each parameter. In each figure, (c) shows a schematic illustrating the particular parameter being adjusted. 

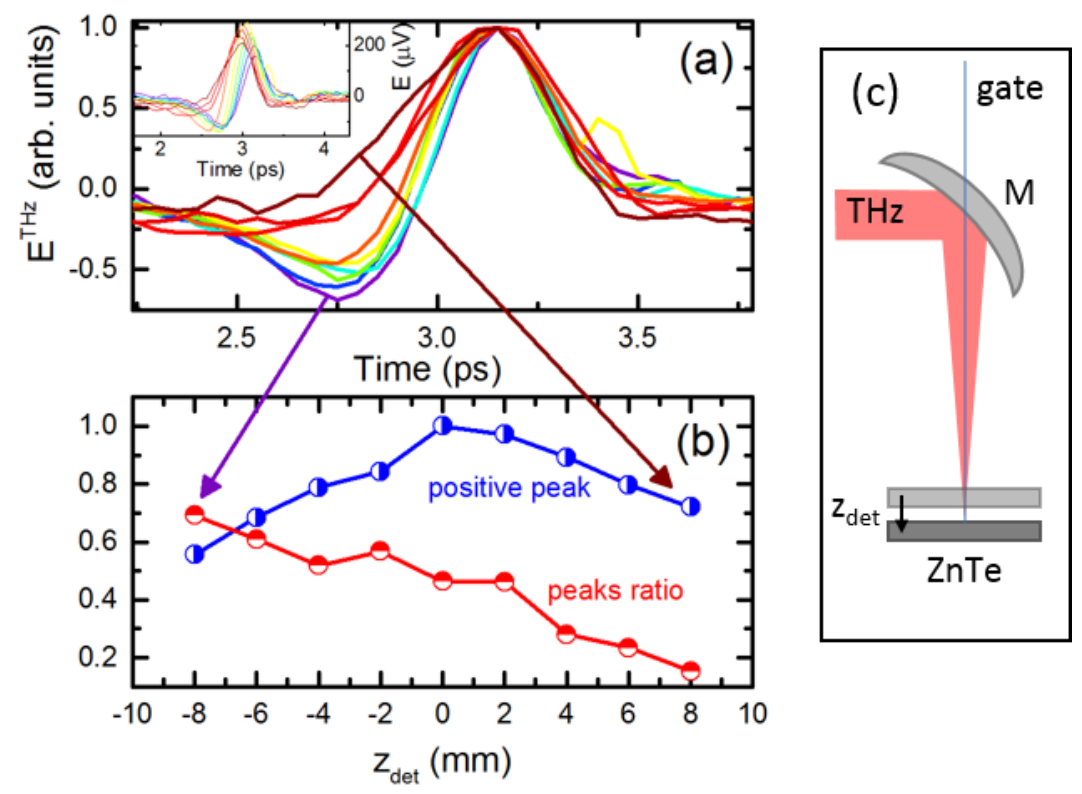

Figure 2.7: The effect of moving the ZnTe EO crystal along the optical $z$-axis. (a) Normalized $\mathrm{THz}$ shape as a function of last parabolic mirror angle. The inset shows the raw data before normalization. (b) The effect on peak strength and shape. (c) Schematic illustrating $z_{d e t}$.

In the case of each individual parameter, there is a peak in the (b) blue trace for which the signal can be said to be "optimized". However, the (b) red trace does not show an extreme at the same location. This demonstrates the importance of optimization of the detection optics: If the detection is adjusted to optimize the first (negative) peak, the same configuration results in a weaker second (positive) peak! Shown here are five parameters (ZnTe z-position, last parabolic mirror horizontal and vertical tilt, and gate mirror horizontal and vertical tilt) which all need to be optimized simultaneously. In addition to these, there are additional degrees of freedom: The $x, y$, and $z$ position of the last parabolic mirror, and the position and tilt of the first three parabolic mirrors.

Therefore, for a given cone of $\mathrm{THz}$ radiation, there are at least 23 degrees of freedom that must be simultaneously optimized to obtain an exact replica of a $\mathrm{THz}$ transient excited by or transmitted through a sample. Any of these degrees of freedom being slightly incorrect results in a drastic change in the shape of the THz pulse that is measured. The most effective example of this seems to be the horizontal tilt of the last parabolic mirror (Figure 2.8 below). $\mathrm{A}<1 \mathrm{~mm}$ shift of the $\mathrm{THz}$ image on the $\mathrm{ZnTe}$ results in a twofold increase in the ratio between the peaks. 

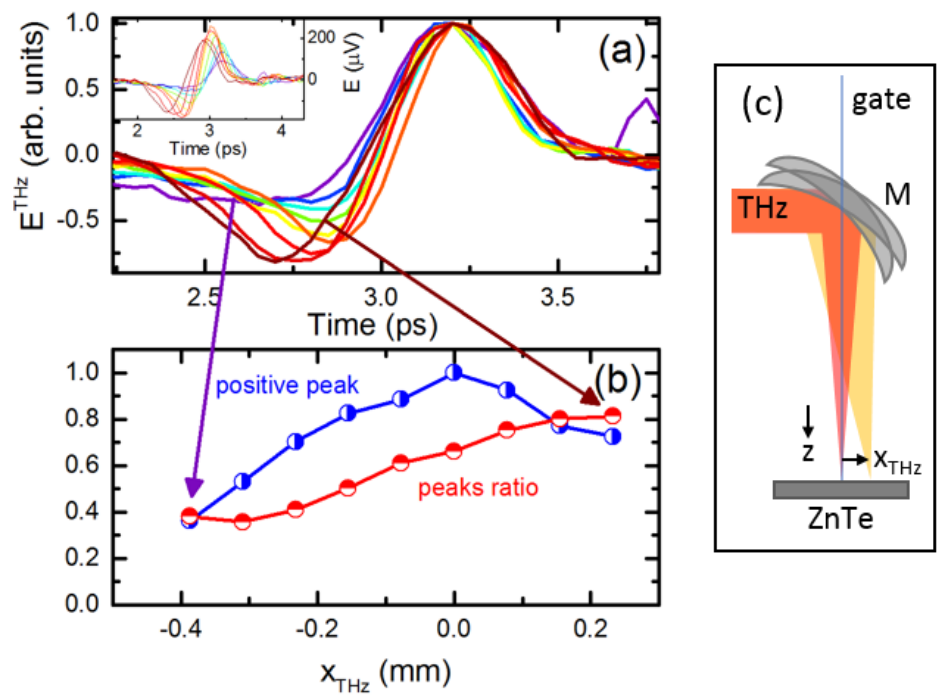

Figure 2.8: (a) Normalized THz shape as a function of last parabolic mirror angle. The inset shows the raw data before normalization. The angle of the parabolic mirror is adjusted in the $x z$ plane such that the image position on the $\mathrm{ZnTe}$ is shifted by $x_{T H z}$, with 0 indicating the position with peak signal. (b) The effect on peak strength and shape. (c) Schematic illustrating $x_{T H z}$.

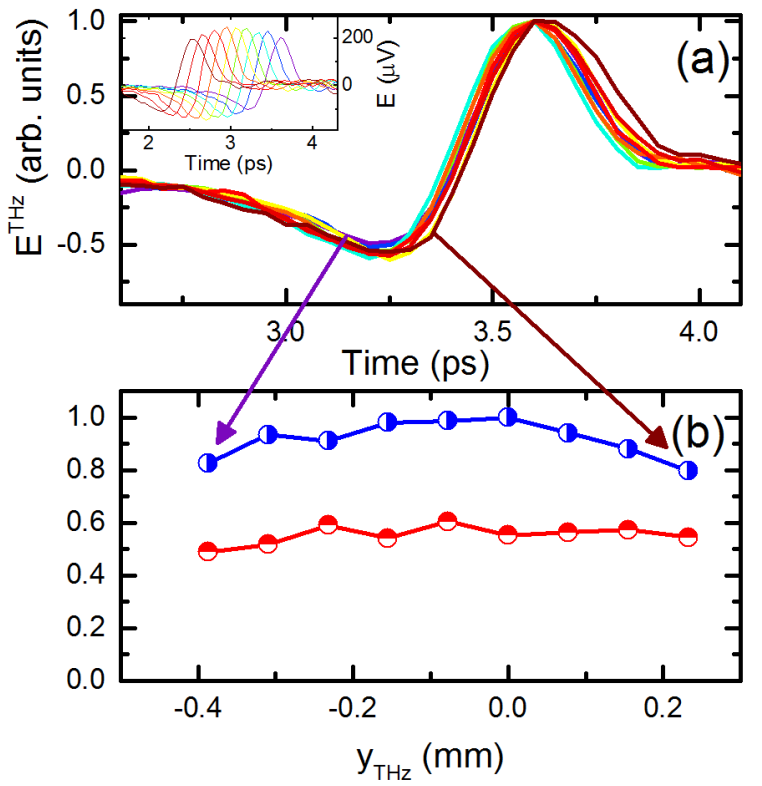

Figure 2.9: (a) THz shape as a function of last parabolic mirror angle. The inset shows the raw data before normalization. The angle is adjusted in the $y z$-plane such that the image position on the $\mathrm{ZnTe}$ is shifted by $y_{T H Z}$ (out of the page in Figure 2.8(c)), with 0 indicating the position with peak signal. (b) The effect on peak strength and shape. 


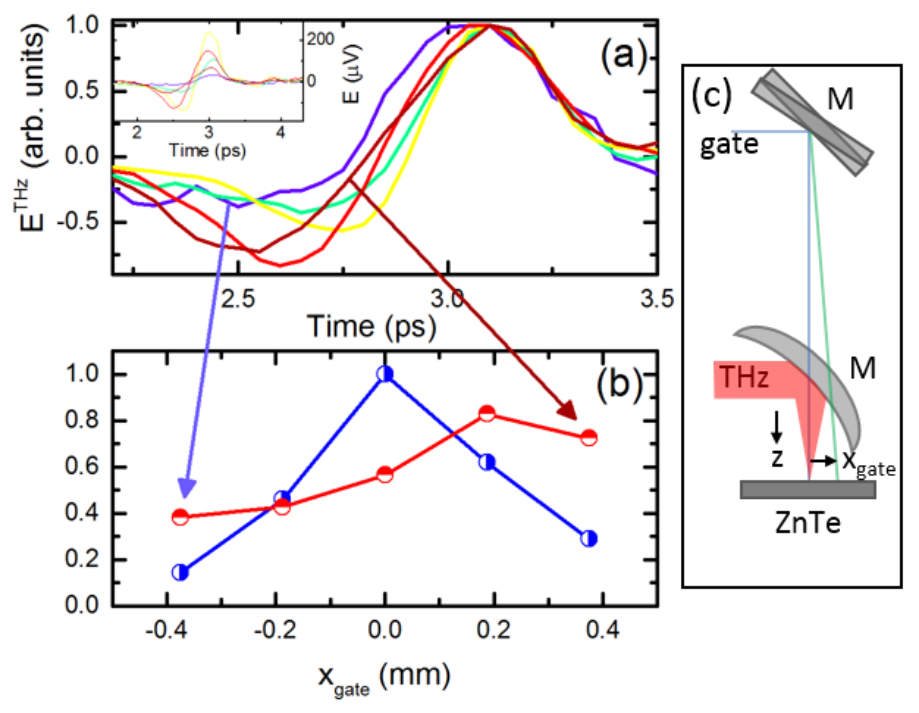

Figure 2.10: (a) THz shape as a function of gate mirror angle. The inset shows the raw data before normalization. The angle of the mirror is adjusted in the $x z$-plane such that the gate pulse on the $\mathrm{ZnTe}$ is shifted by $x_{\text {gate }}$, with 0 indicating the position with peak signal. (b) The effect on peak strength and shape. (c) Schematic illustrating $x_{\text {gate }}$.

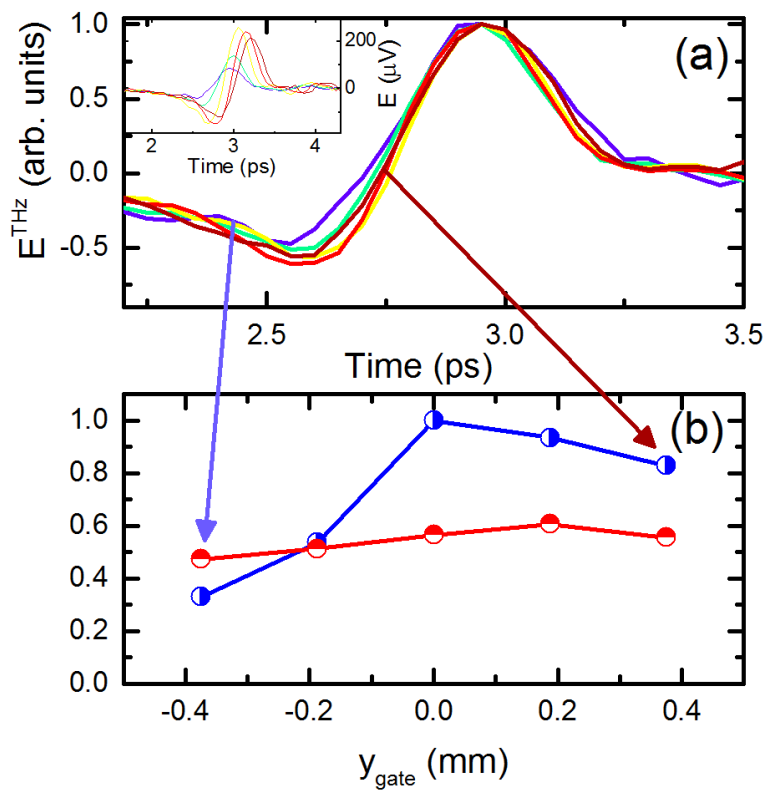

Figure 2.11: (a) THz shape as a function of gate mirror angle. The inset shows the raw data before normalization. The angle is adjusted in the $y z$-plane such that the gate pulse on the $\mathrm{ZnTe}$ is shifted by $y_{\text {gate }}$ (out of the page in Figure 2.10(c)), with 0 indicating the position with peak signal. 


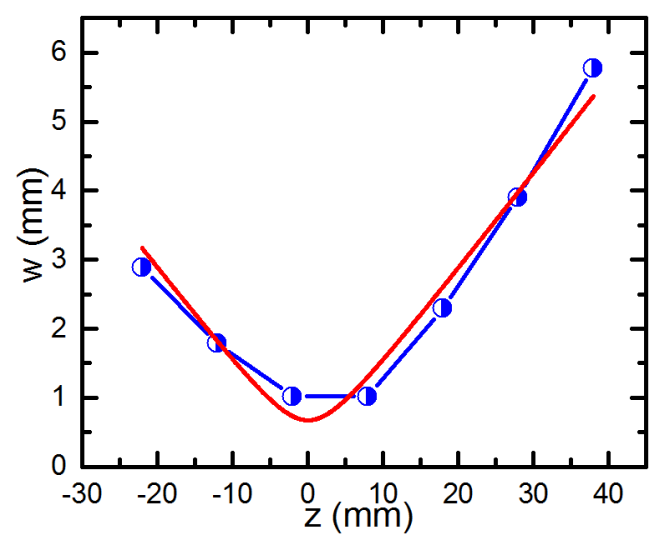

Figure 2.12: $1 / e^{2}$ beam waist as a function of $z$-position at the intermediate focus of the parabolic mirrors. The blue is experimental data and the red curve is a gaussian beam fit. The fit is imperfect because it assumes a symmetry about the focal point, but in the real data misalignment causes a break in this symmetry.

For size comparison, Figure 2.12 shows the shape of the $\mathrm{THz}$ pulse at the intermediate focus. The blue data points were measured by placing an iris at the intermediate focus and closing it until the signal measured was cut approximately in half. The iris diameter was measured, giving the FWHM, which was then converted to the $1 / e^{2}$ width $w$ using $2 w=\sqrt{2} F W H M / \sqrt{\ln 2}$. The red curve is a fit based on the gaussian beam width $w(z)=w_{0} \sqrt{1+\left(z / z_{R}\right)^{2}}$ with the fitting parameters being the waist diameter $w_{0}=0.67 \mathrm{~mm}$ and the Rayleigh range $z_{R}=4.81 \mathrm{~mm}$. These parameters give a wavelength $\lambda=0.3 \mathrm{~mm}$ which corresponds to $1 \mathrm{THz}$. The asymmetry in the data is probably due to slight misalignment of the first two parabolic mirrors, which can distort the wavefront resulting in the transient shape changes shown above. These distortions are significant even when the $x$ and $y$ adjustments are a fraction of the beam waist, or the $z$ adjustments are a fraction of the Rayleigh range. 


\section{5: Comparing multiple transients}

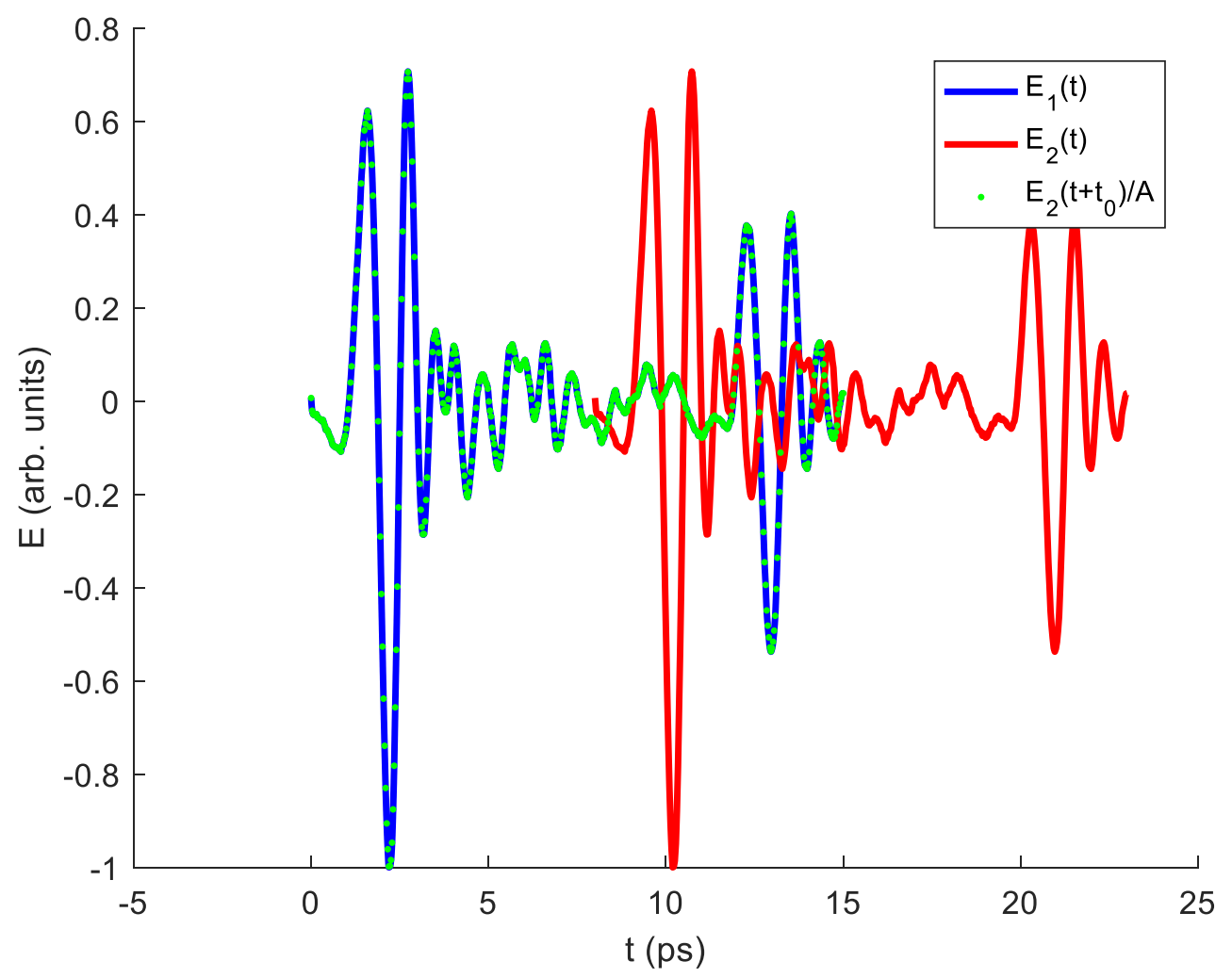

Figure 2.13: The effect of a non-dispersive refractive element in the $\mathrm{THz}$ beam path. Here, $E_{2}$ is shifted by $t_{0}=8 \mathrm{ps}$.

In many experiments it is useful to compare $\mathrm{THz}$ transients to each other. In this section we discuss methods of comparing two fields labeled $E_{1}(t)$ and $E_{2}(t)$. In some cases, $E_{2}(t)$ may be offset such that $E_{1}(t)=E_{2}\left(t+t_{0}\right)$, due to an optic element with a significant refractive index $n$ and thickness $d$ added to the path of $E_{2}$, but not $E_{1}$. In this case, the relative time delay of $E_{2}$ is

$$
t_{0}=\frac{d}{c}\left(n-n_{\text {air }}\right)
$$

where $n_{\text {air }}=1$ is the refractive index of air. Figure 2.13 illustrates an example of this effect.

In other cases, $E_{2}$ may be uniformly scaled relative to $E_{1}$ due to the addition of an absorptive, nondispersive element of thickness $d$ to the path of $E_{2}$, such that $E_{1}=E_{2} / A$. In this case, 
where $\alpha$ is the absorption coefficient of the added element. Figure 2.14 illustrates an example of this case.

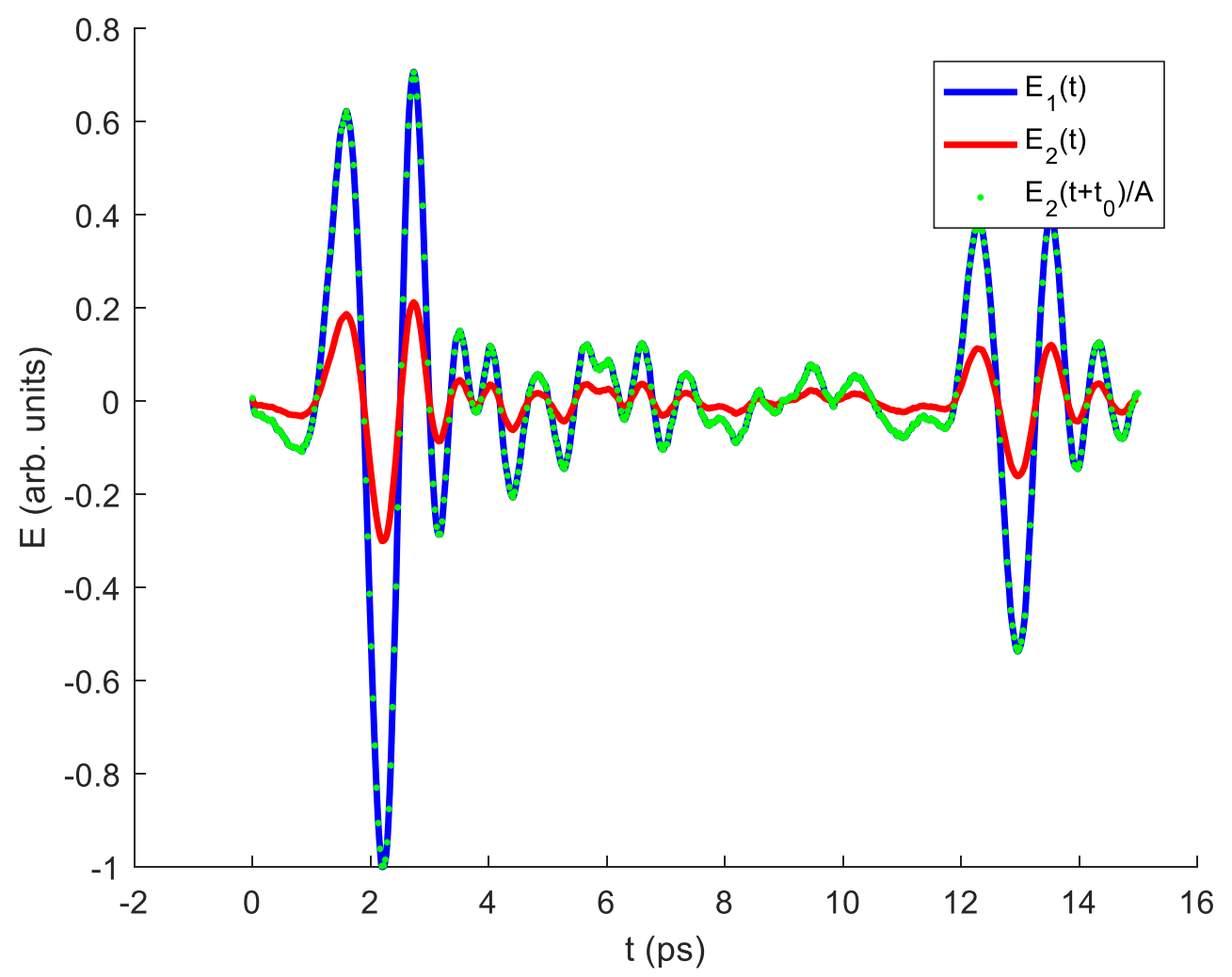

Figure 2.14: The effect of a non-dispersive absorptive element in the beam path. $E_{2}$ is attenuated by a factor of $A=0.3$.

Of course, in most real experiments, both $A$ and $t_{0}$ are present simultaneously. Many media do have non-negligible dispersion, and often the added dispersion significantly changes the shape of the $\mathrm{THz}$ pulse. To take this into account, a script was created using Matlab to simultaneously adjust $A$ and $t_{0}$ and calculate the resulting coefficient of determination, $R^{2}$, which is a method of measuring how similar two curves are to each other. When comparing the shifted and scaled $E_{3}=E_{2}\left(t+t_{0}\right) / A, R^{2}$ is calculated using

$$
R^{2}=1-\frac{S S_{\text {res }}}{S S_{\text {tot }}}
$$


where $S S_{\text {res }}=\sum_{t}\left(E_{3}(t)-E_{1}(t)\right)^{2}$ is the residual sum of squares and $S S_{t o t}=\sum_{t}\left(E_{3}(t)-\bar{E}_{3}(t)\right)^{2}$ is the total sum of squares. $\bar{E}_{3}$ is the mean of $E_{3}$. Hence, when $R^{2}=1$, the two curves being compared are identical.

The contour plot in Figure 2.15 shows the maximum value of $R^{2}$ that can be obtained in adjusting $A$ and $t_{0}$. For this condition, $A=0.7443$ and $t_{0}=0.624 \mathrm{ps}$.

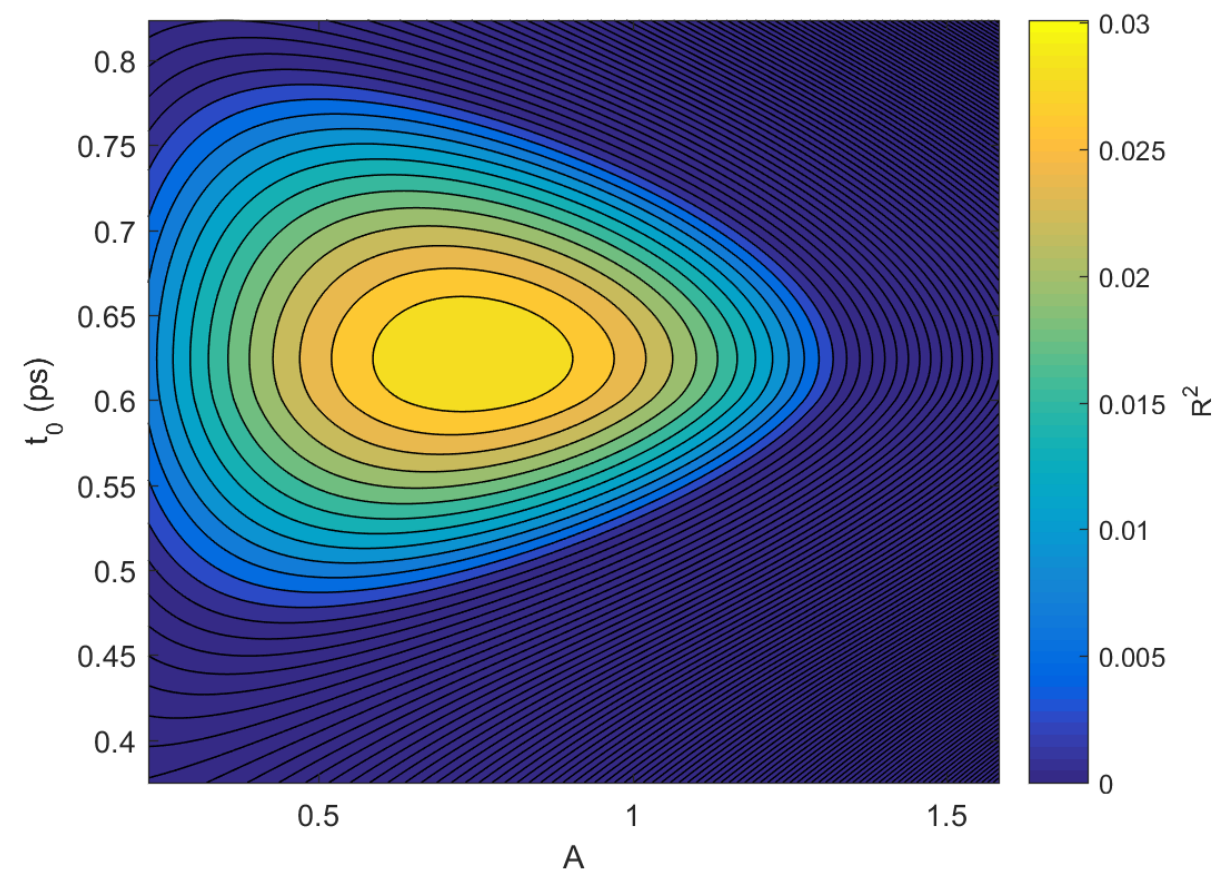

Figure 2.15: Resulting $R^{2}$ values from the adjustment of $A$ and $t_{0}$. The maximum $R^{2}=0.03$ occurs when $A=0.7443$ and $t_{0}=0.624$. 


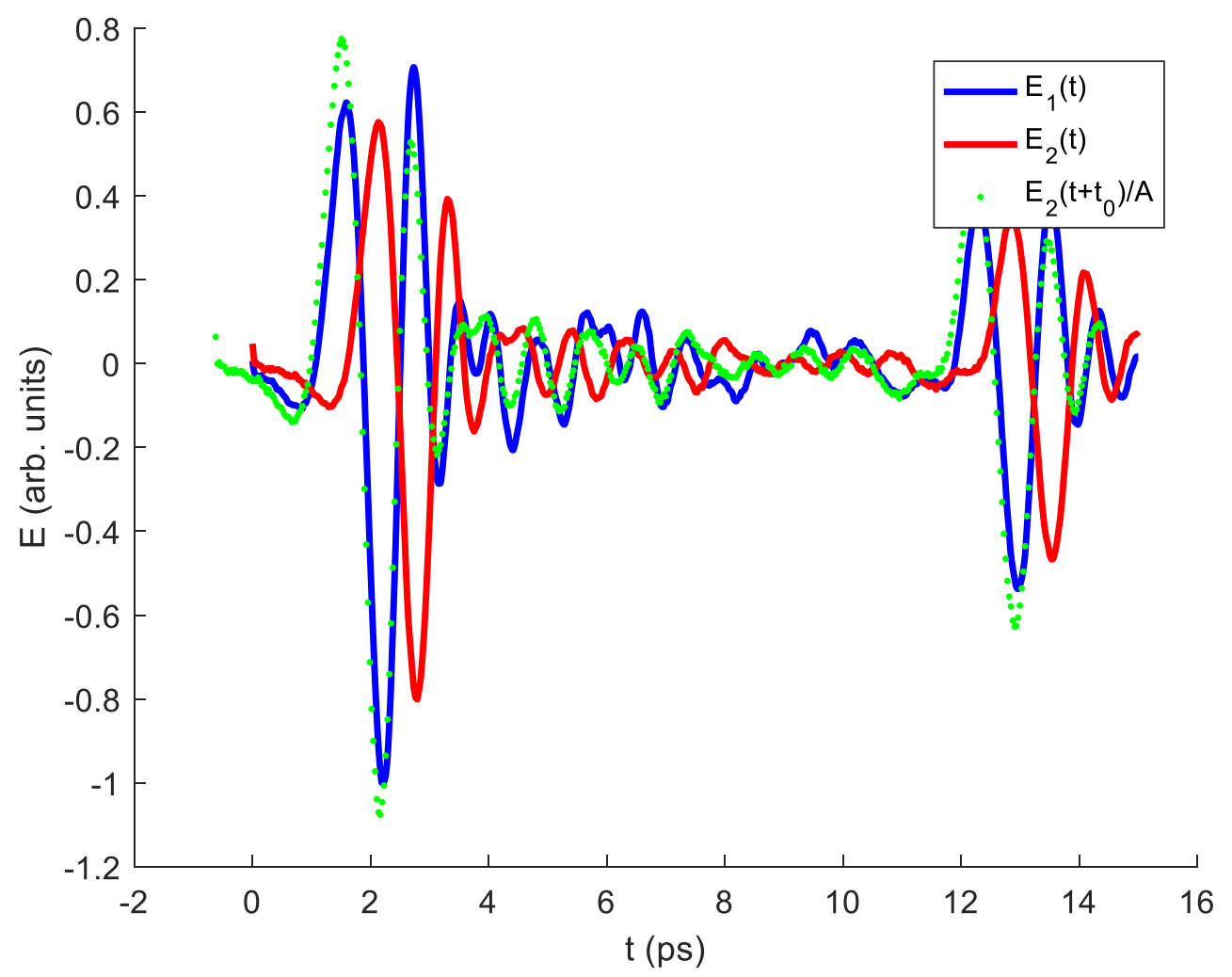

Figure 2.16: The result of shifting and scaling $E_{2}$ to achieve the best possible overlap with $E_{1}$.

Figure 2.16 shows the result of shifting and scaling $E_{2}$ to compare it with $E_{1}$. From this it becomes apparent that the relative weight of the main peaks changes. In $E_{2}$, the bulk of the energy arrives early on, and it attenuates more quickly than in $E_{1}$. The same behavior is apparent in the reflection which occurs at $\sim 13$ ps as well.

This method can be generally applied to any two THz pulses. For example, it is used to compare arrival times of pulses under different conditions in Section 3.3.2. However, for drastic pulse shape changes where a high $R^{2}$ value cannot be achieved, it is limited in its effectiveness. 


\section{Chapter 3: THz-TDS of Antiferromagnetic Magnons}

AF materials are promising for spintronic applications due to their ability to exhibit a magnetic order without a macroscopic magnetization. If an AF device were to store information in its magnetic order, the device would have no magnetic interaction with its neighbors even in close proximity. AFs interact strongly with radiation in the far-IR/THz frequency range due to their characteristic $\mathrm{AF}$ exchange energies. ${ }^{83}$ Therefore, THz can be a valuable tool in studying the magnons in AFs.

Several methods have been used to study resonances in AF materials, including antiferromagnetic resonance, ${ }^{84}$ Fourier transform infrared spectroscopy, ${ }^{85}$ neutron scattering, ${ }^{86-89}$ nuclear magnetic resonance, ${ }^{90,91} \mathrm{x}$-ray absorption, ${ }^{92,93}$ and Raman spectroscopy ${ }^{94-96}$ Until recently, optical techniques have only been employed in the linear regime; however, with the availability of ultrafast pulsed lasers, it has become possible to study AF magnons and coherently control them using $\mathrm{THz}$ pulses, ${ }^{97-100}$ allowing ultrafast switching in spintronic devices.

Impressive coherent control over spin waves has been demonstrated using femtosecond laser pulses in the room-temperature AF nickel oxide (NiO), ${ }^{98}$ in which spins were manipulated using the Zeeman torque $G=\gamma S \times B$, where $\gamma$ is the gyromagnetic constant, $S$ is the spin of an electron, and $B$ is a timedependent magnetic field (in this case, produced by a laser pulse). NiO has a rock-salt structure with inversion symmetry. It has the advantage of a relatively high Néel temperature of $T_{N}=523 \mathrm{~K}$, below which spins are ferromagnetically aligned within the (111) planes. ${ }^{101}$

Although room-temperature AFs like $\mathrm{NiO}$ are more readily used in coherent control techniques, the magnon temperature dependence is more easily studied in a well-characterized low-temperature AF material like manganese fluoride $\left(\mathrm{MnF}_{2}\right)$. In the past, $\mathrm{MnF}_{2}$ has been studied as a model $\mathrm{AF}$ with and without doping. ${ }^{102,103}$ Absorption spectroscopy has also been used to study the D bands in UV photon energies. ${ }^{104,105} \mathrm{MnF}_{2}$ has a rutile crystal structure ${ }^{106}$ with a spin lattice that is $\mathrm{AF}$ below the Néel temperature $T_{N}=67.33 \mathrm{~K} \cdot{ }^{107}$ The magnetic order comes from the $\mathrm{Mn}^{2+}$ ions, which have electron configuration $[A r] 4 s^{0} 3 d^{5}$. Since the $3 d$ shell is half full, the ions have spin angular momentum $S=5 / 2$, but orbital angular momentum $L=0$, and thus no spin-orbit coupling. Previous studies of its spin structure used magnetic 
neutron scattering. ${ }^{108}$ Recently, a study has been reported of exchange bias in a device made from $\mathrm{MnF}_{2}$ in contact with Fe. ${ }^{109}$ Other studies reported localized control of spin states via linearly polarized laser stimulus $^{110}$ as well as demonstrated the spin Seebeck effect correlated with the spin-flop transition in $\mathrm{MnF}_{2 .}{ }^{111}$ This work continues to demonstrate the viability of materials like $\mathrm{MnF}_{2}$ in spintronic devices.

THz-TDS provides a novel method which can be used for all-optical switching and control of magnonic states in $\mathrm{AF}$ materials like $\mathrm{MnF}_{2}$. THz-TDS was used to examine the one-magnon resonance in single crystal $\mathrm{MnO}, 112$ but it is important to extend these techniques to the well-characterized model system, $\mathrm{MnF}_{2}$, for which the observed results can be well-described by simple models such as mean-field theory. Here we discuss our observations of the one-magnon and two-magnon resonances in $\mathrm{MnF}_{2}$. We then compare our results with those obtained using alternative techniques and with theoretical predictions. We demonstrate how THz-TDS can be used to analyze resonance frequencies and linewidths as a function of temperature, as well as changes in refractive index caused by sublattice magnetization, thermal lattice expansion, and hyperfine interactions. Thus, we lay a foundation for a methodology to manipulate spin waves in AF materials.

\section{1: Sample characterization}

The single-crystal $\mathrm{MnF}_{2}$ sample was grown at the University of California, Santa Barbara using the Bridgemann-Stockbarger technique. ${ }^{113}$ The sample used is cylindrical in shape, $10 \mathrm{~mm}$ in diameter and $1.9 \mathrm{~mm}$ thick, with the $c$-axis oriented perpendicular to the face of the sample. The two faces of the sample were polished for optical transparency. The stoichiometry of our sample was tested using x-ray photoelectron spectroscopy (XPS). Prior to XPS measurements, the sample surface was sputtered with $\mathrm{Ar}$ ions to clean off surface contaminants. The XPS results in Figure 3.1 confirmed the sample composition of $\mathrm{Mn}^{2+}$ and $\mathrm{F}^{-}$, closely matching what has been measured previously. ${ }^{114}$ 


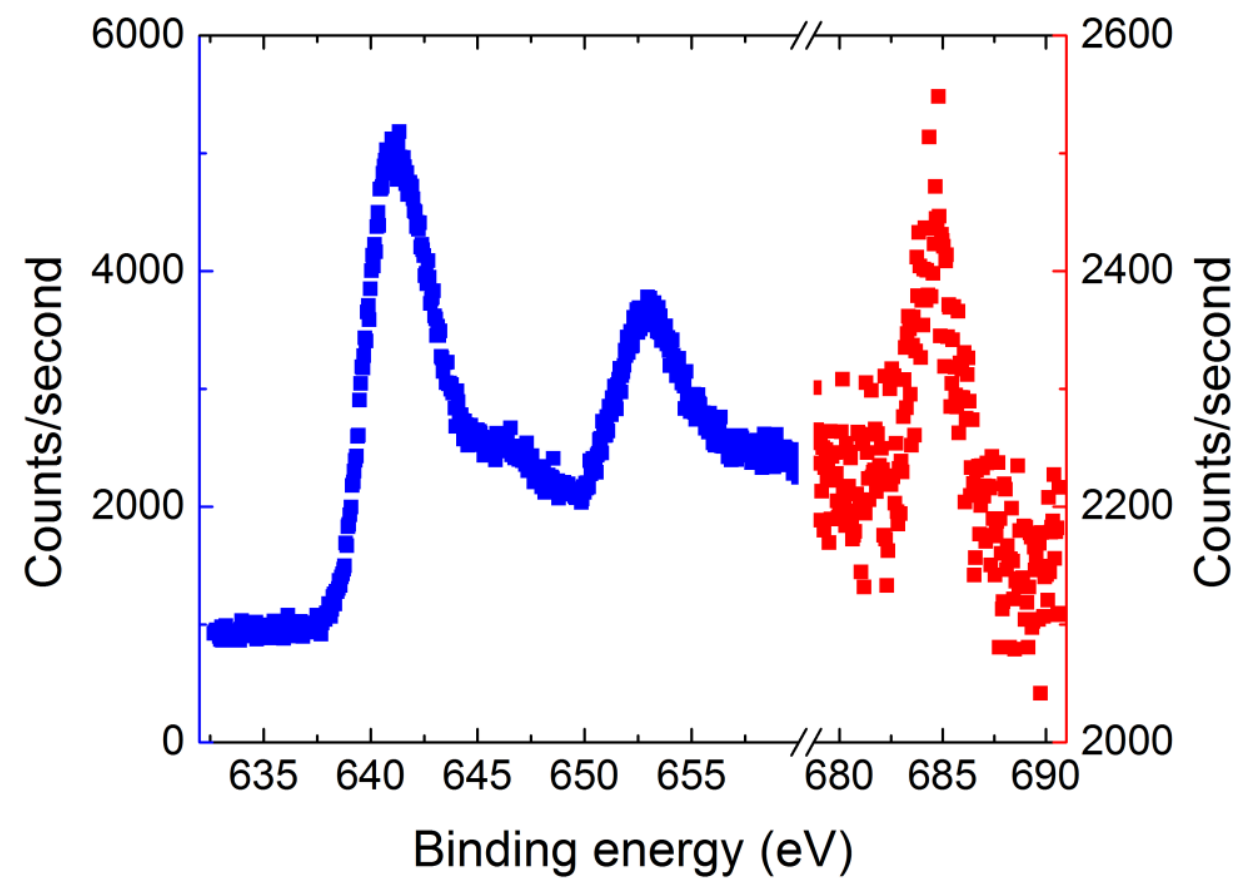
axis).

Figure 3.1: XPS results indicating sample composition of $\mathrm{Mn}^{2+}$ (blue, left axis) and $\mathrm{F}^{-}$(red, right

The out-of-plane crystal (001) orientation was confirmed using a four-axis goniometer x-ray diffraction system (Rigaku RU-300) with a Cu rotating anode. Therefore, the magnetic easy axis was oriented out of the single crystal plane and parallel to the $\mathrm{THz}$ wave vector. ${ }^{108}$

Magnetization measurements were performed using a commercial magnetometer with superconducting quantum interference device sensor (MPMS-SQUID from Quantum Design). The magnetic moment was measured as a function of temperature $T$ in a magnetic field of 100 Oe upon cooling (field cooled or FC), and upon heating after cooling in zero-field (ZFC). As shown in Figure 3.2 in which the magnetic moment was converted to inverse susceptibility $\chi^{-1}=H / M$, no splitting between FC and ZFC curves was found, indicating absence of uncompensated magnetization. The data showed paramagnetic behavior for $T>T_{N}$ and AF behavior for $T<T_{N}$, with a peak indicating $T_{-} N=68 \pm 1 \mathrm{~K}$, in agreement with bulk $\mathrm{AF}$ behavior of $\mathrm{MnF}_{2}$. 
Figure 3.2 shows that the linear behavior of $\chi^{-1}$ for $T>T_{N}$ can be fit to the Curie-Weiss law for an antiferromagnet,

$$
\chi^{-1}=\frac{T+\theta}{C}
$$

where $C=2.08 \mathrm{~K}$ and $\theta=-90.2 \mathrm{~K}$, in good agreement with previously measured values for $\mathrm{MnF}_{2 .}{ }^{115}$

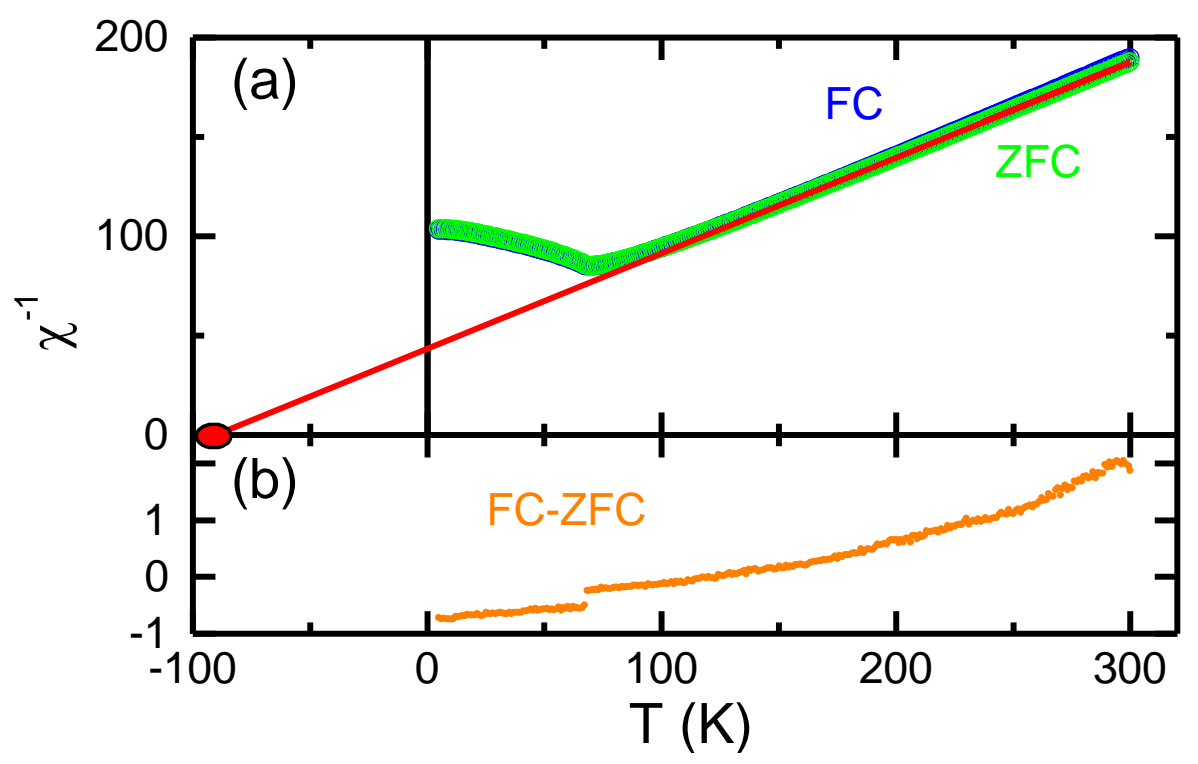

Figure 3.2: (a) Inverse susceptibility as a function of temperature measured in a field $H=100$ Oe. The field cooled (FC- blue) and zero-field cooled (ZFC- green) curves show good overlap. The red is a linear fit in the high temperature region which intercepts $\chi^{-1}=0$ at $\theta=-90.2 \mathrm{~K}$. (b) Residual FC-ZFC.

\section{2: Experiment and analysis}

The $\mathrm{THz}$ radiation is generated and detected as described in Section 2.1. The $\mathrm{THz}$ is then passed through a sapphire window into the cryostat, through the sample, then through the second sapphire window out of the cryostat, as illustrated in Figure 3.3. 


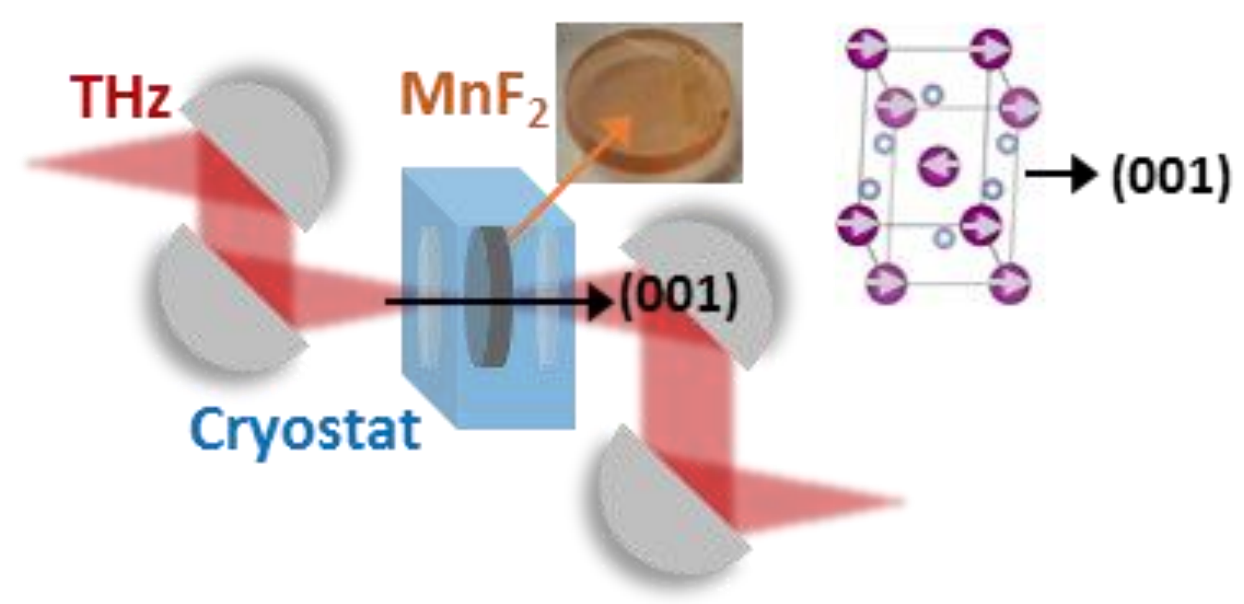

Figure 3.3: Schematic of $\mathrm{THz}$ transmission experiments with a photograph of the $\mathrm{MnF}_{2}$ sample. Upper-right inset shows the atomic and spin structure with crystal (001) vector pointing to the right; purple and gray spheres are $\mathrm{Mn}$ and $\mathrm{F}$ atoms, respectively. $\mathrm{THz}$ is generated and detected in the same way as described in Figure 2.1.

Time-resolved traces were measured by varying the delay time $t$ of the gate pulse with respect to the $\mathrm{THz}$ pulse. Then, spectra were obtained by taking Fourier transforms of the time-domain data. A window function cuts off at $10 \mathrm{ps}$ after the positive maximum to eliminate contributions from the reflection of the pump pulse which occurs $12 \mathrm{ps}$ after the positive maximum. This window allows a spectral resolution of $\sim 0.1 \mathrm{THz}$.

The reference spectrum $\hat{E}_{r e f}(v)$ shown in Figure 3.4(a) was obtained at room temperature by following the above procedure without the sample in the beam path. The limited bandwidth of the detection used prevents a complete analysis at high frequencies. The curve includes known water absorption valleys at 1.7 and $1.9 \mathrm{THz} .{ }^{77}$ Above $\sim 2.3 \mathrm{THz}$, the reference spectrum is weak mainly due to transmission of the cryostat windows, strongly decreasing the signal to noise ratio. This high-frequency tail could be improved in the future by using a different pump crystal, EO sampling crystal, and cryostat windows which allow higher transmission at the desired frequencies.

The sample was then cooled from room temperature to $T=6.2 \mathrm{~K}$. The transmitted spectrum of the frequency-dependent electric field amplitude $\left|\hat{E}_{T}\right|(v)$ was recorded, and the differential transmission was calculated using

$$
\frac{\Delta|\hat{E}|}{\left|\hat{E}_{r e f}\right|}=\frac{\left[\left|\hat{E}_{r e f}\right|(v)-\left|\hat{E}_{T}\right|(v)\right]}{\left|\hat{E}_{r e f}\right|(v)}
$$


The result is the blue curve in Figure 3.4(b). The shaded region represents the noise in the measurement as a function of frequency; as the magnitude of $\left|\widehat{E}_{\text {ref }}\right|$ decreases, the signal grows noisier.

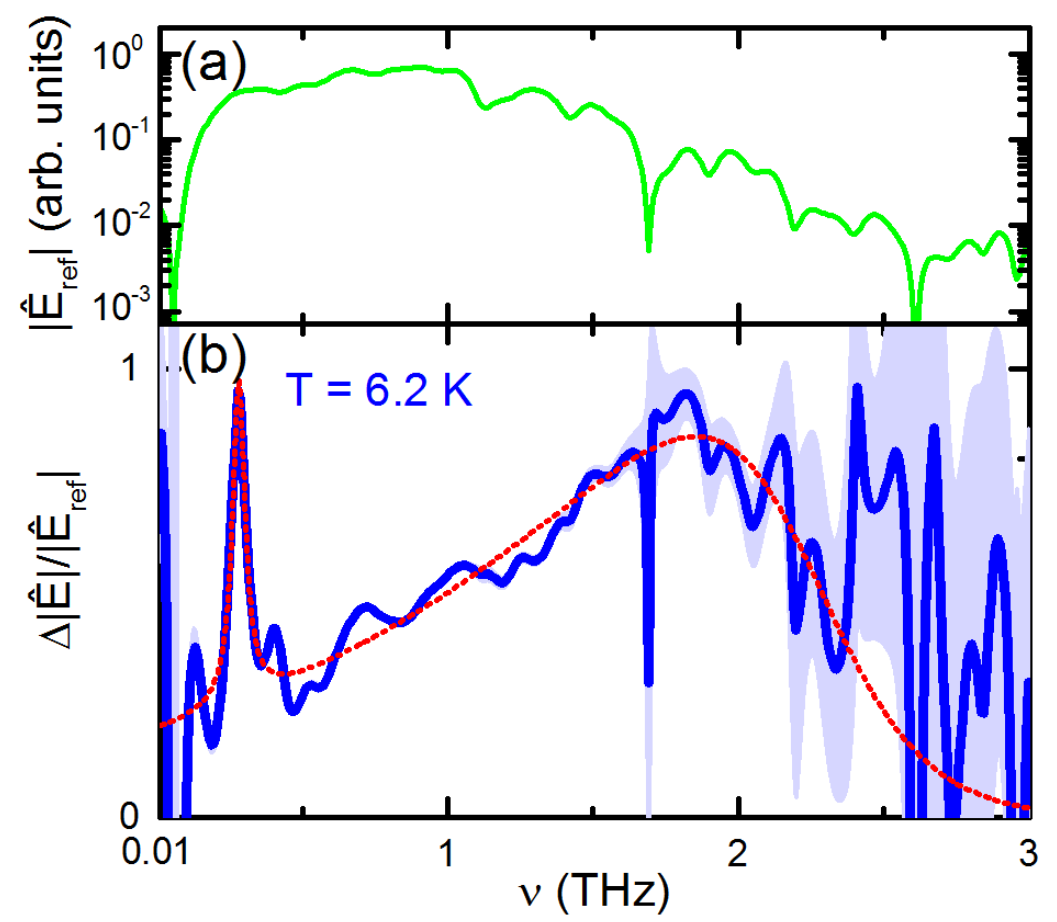

Figure 3.4: (a) Reference THz spectrum. (b) Differential transmission $\Delta|\hat{E}| /\left|\hat{E}_{\text {ref }}\right|$ at $T=6.2 \mathrm{~K}$ data (blue curve) and a fit of two peaks (red dotted curve, see Equation 3.3. The shaded region shows the uncertainty as a function of $v$.

The two main features in the differential transmission were fitted with peak functions such that

$$
\begin{gathered}
\frac{\Delta|\hat{E}|}{\left|\hat{E}_{r e f}\right|}=A^{(1)}\left[\frac{\pi v_{F W H M}^{(1)}}{2}+\left(\frac{2\left(v-v_{0}^{(1)}\right)}{v_{F W H M}^{(1)}}\right)^{2}\right]^{-1} \\
+A^{(2)}\left[1+\exp \left(-\frac{v-v_{0}^{(2)}}{v_{w 1}^{(2)}}\right)\right]^{-1}\left(1-\left[1+\exp \left(-\frac{v-v_{0}^{(2)}}{v_{w 2}^{(2)}}\right)\right]^{-1}\right) .
\end{gathered}
$$

The first term describes the one-magnon peak as a Lorentzian with amplitude $A^{(1)}$, center frequency $v_{0}^{(1)}$, and full-width at half maximum $v_{F W H M}^{(1)}$ as fitting parameters. The second term describes the two-magnon peak as an asymmetric double sigmoidal with amplitude $A^{(2)}$, center frequency $v_{0}^{(2)}$, and full-width at half 
maximum $v_{F W H M}^{(2)}=v_{w 1}^{(2)}+v_{w 2}^{(2)}$ as fitting parameters. The two-magnon resonance is expected to be asymmetric because the signal reflects the magnon DOS near the zone boundary. ${ }^{116}$ The fit to Equation 3.3 is shown in Figure 3.4(b) as the dotted red curve. Data in the range $v>\sim 2.3 \mathrm{THz}$ was deemed too noisy and thus was not included when optimizing the fitting function. This experiment and fitting procedure was repeated at increasing temperatures up to $T=295 \mathrm{~K}$ to obtain the plot in Figure 3.6. The temperature dependence of the extracted fitting parameters is discussed in Section 3.3.1.

In addition to this spectrum analysis, we used the $\mathrm{THz}$ transients in Figure 3.5 to extract the pulse velocities. The largest peak of each transient was fitted by the derivative of a Gaussian,

$$
E=-A\left(t-t_{0}\right) e^{-B\left(t-t_{0}\right)^{2}}
$$

where $A$ and $B$ are arbitrary scaling factors and $t_{0}$ is the delay time of the pulse. The $t$ axis is defined such that the fit of the reference pulse $E_{\text {ref }}$ results in $t_{0}=0$. An example of this fit is shown in the inset of Figure 3.5 .

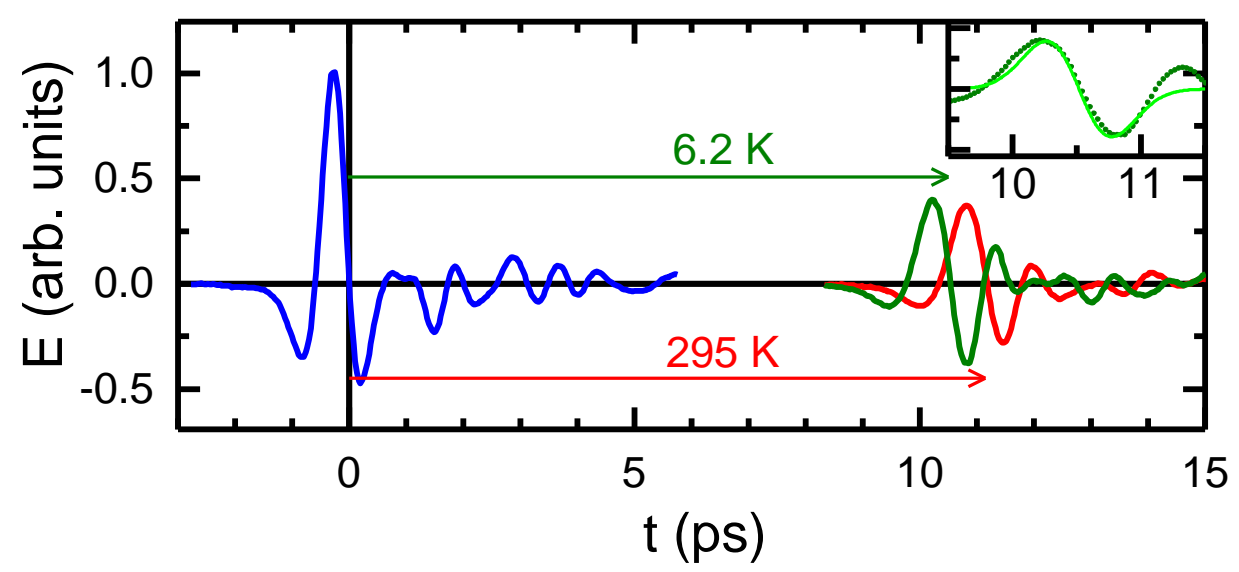

Figure 3.5: Temperature dependence of the timing of transmitted $\mathrm{THz}$ transients. Comparison of the reference pulse traveling through air (blue) with the pulse traveling through the sample at $295 \mathrm{~K}$ (red) and $6.2 \mathrm{~K}$ (green). The change in delay time of the pulse corresponds to changes in refractive index. The inset shows an example fit of Equation 3.4 (light green) to the $T=6.2 \mathrm{~K}$ data (dark green).

The thickness $L(T)$ of the sample was calculated using the measured room temperature value $L=1.9 \mathrm{~mm}$ and the relation $\Delta L / L=\alpha \Delta T$, where $\alpha$ is the linear thermal expansion coefficient of $\mathrm{MnF}_{2}$ along the $c$-axis ${ }^{117}$. The change in thickness over the entire temperature range was calculated to be $0.31 \%$. 
The temperature-dependent $\mathrm{THz}$ refractive index $n(T)$ was obtained for each pulse via the relation $t_{0}(T)=L(T)\left[n(T)-n_{\text {air }}\right] / c$. Here, $c$ is the speed of light and $n_{\text {air }}=1$ is the refractive index of air. The room temperature refractive index was calculated to be 2.76 and over the entire temperature range it changes by 3.4\%. These results are discussed in Section 3.3.2.

\section{3: Discussion of results}

\subsection{1: Magnon resonance frequency}
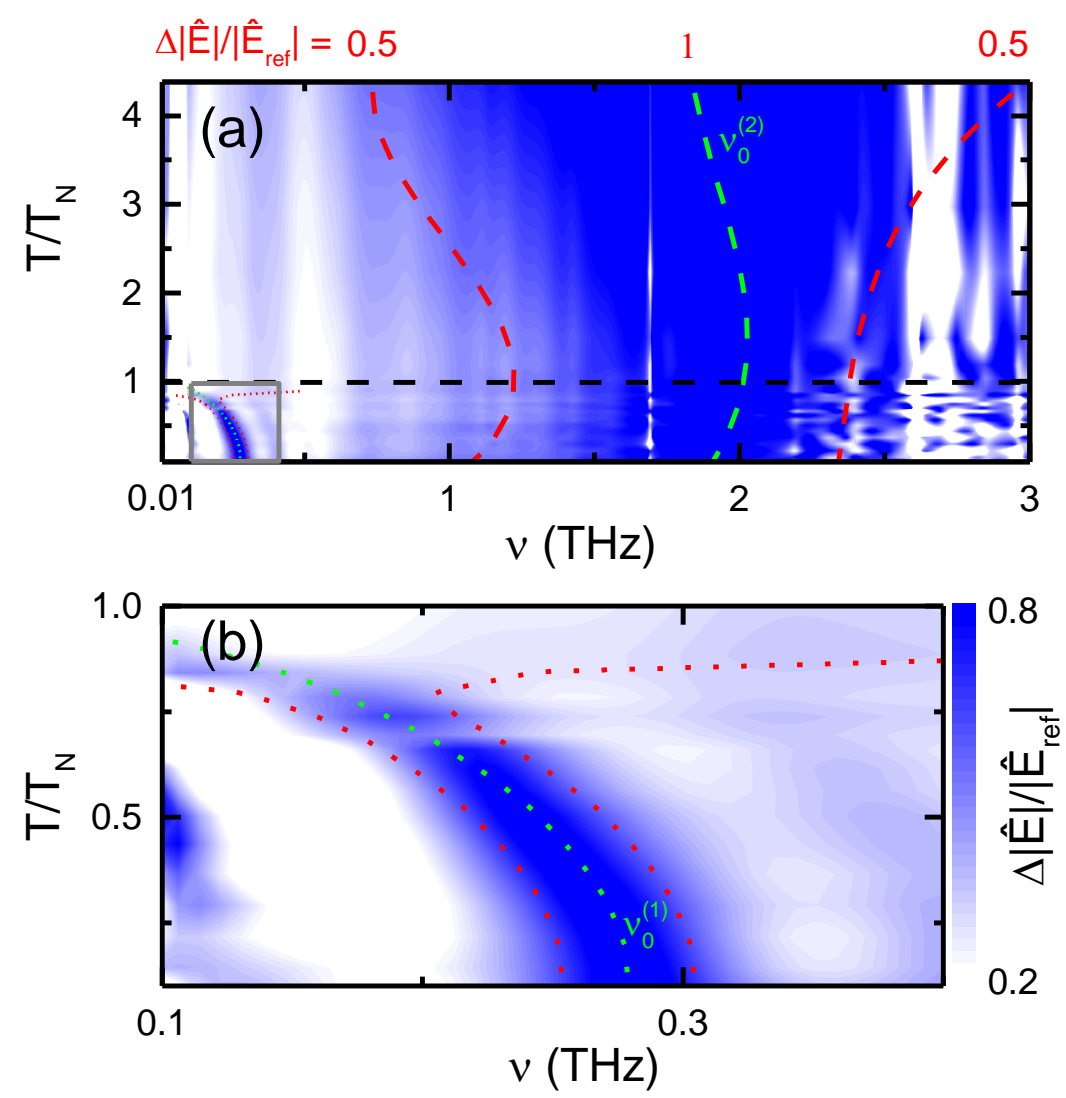

Figure 3.6: (a) Differential transmission over all available temperatures and frequencies. Two distinct features are highlighted by the curves shown as guides to the eye, approximated from the results of the Equation 3.3 fit. The one-magnon feature $v_{0}^{(1)}$ bounded by the gray rectangle has a maximum at the green dotted line, and drops off by half when it reaches the red dotted lines on either side. The two-magnon feature $v_{0}^{(2)}$ has a maximum at the green dashed line, and drops off by half when it reaches the red dashed lines on either side. The dashed black line indicates the Néel temperature. (b) Magnified view of the differential transmission in the one-magnon region. The colorbar refers to both (a) and (b). 
Two distinct features are visible in the transmitted spectrum $\Delta|\hat{E}| /\left|\hat{E}_{r e f}\right|$ in Figure 3.6. We first discuss the low-frequency feature, which we show magnified in Figure 3.6(b). At $T=6.2 \mathrm{~K}$, this feature has a narrow peak centered at $0.28 \mathrm{THz}$ with a FWHM of $\sim 0.053 \mathrm{THz}$. We attribute this feature to the onemagnon resonance.

Figure 3.7(a) shows the temperature dependence of the one-magnon resonance frequency $v_{0}^{(1)}$, which is taken from the fit of Equation 3.3. The resulting curve shows typical behavior for $v_{0}$, gradually redshifting as the sample temperature $T$ increases, and disappearing as the Néel temperature is reached.

The magnon resonance frequency $v_{0}^{(1)}$ is given by

$$
v_{0}^{(1)}=\left[v_{A}\left(2 v_{E}+v_{A}\right)\right]^{1 / 2}
$$

where the anisotropy and exchange frequencies, respectively, are given by $v_{A}=g \mu_{B} H_{A}(T) / h$ and $v_{E}=$ $8\left\langle S^{z}\right\rangle J_{E} / h^{118}$ for $\mathrm{MnF}_{2}$ where each $\mathrm{Mn}$ site has 8 nearest neighbors. Here, $g$ is the Landé $\mathrm{g}$-factor, $\mu_{B}$ is the Bohr magneton, $H_{A}$ is the anisotropy field, $h$ is Planck's constant, $\left\langle S^{z}\right\rangle$ is the average $z$-component of the spin angular momentum for each $\mathrm{AF}$ sublattice, and $J_{E}$ is the dominant nearest neighbor exchange. The values of $v_{A}$ and $v_{E}$ are about $0.022 \mathrm{THz}$ and $1.5 \mathrm{THz}$ respectively at $0 \mathrm{~K}$ in $\mathrm{MnF}_{2},{ }^{119,120}$ giving $v_{0}=0.26$ THz with $g=2.0$, which agrees well with our experimental results.

The temperature dependence of $v_{0}$ is a function of $H_{A}(T)$ with $H_{A}(T) \propto\left\langle S^{Z}\right\rangle^{j}$, where $j=1$ or $j=2$ indicates that neighboring spins are completely uncorrelated or completely correlated, respectively, and the real value will lie somewhere in between the two extremes ${ }^{119}$. At low $(T<20 \mathrm{~K})$ and high $(T>50 \mathrm{~K})$ temperature, $j$ has been calculated to be $1.9,{ }^{121}$ indicating strong correlation. However, an accurate value of $j$ in the intermediate temperature range $20 \mathrm{~K}<T<50 \mathrm{~K}$ has not been accurately determined to date.

In mean field theory, $\left\langle S^{z}\right\rangle$ is proportional to the normalized spontaneous sublattice magnetization $M / M_{0}$, which can be described by ${ }^{122}$

$$
M / M_{0}=\mathcal{B}_{J}(x)
$$


where $\mathcal{B}_{J}(x)$ is the Brillouin function. By taking the total angular momentum value for $\mathrm{MnF}_{2}, J=5 / 2$, we obtain

$$
\mathcal{B}_{5 / 2}(x)=\frac{6}{5} \operatorname{coth} \frac{6 x}{5}-\frac{1}{5} \operatorname{coth} \frac{x}{5}
$$

and

$$
x=\frac{15}{7} \frac{M}{M_{0}} \frac{T_{N}}{T} .
$$

Using these expressions, the temperature dependence of $v_{0}$ was modeled for values of $j$ between 1 and 2 with an increment of 0.1 , and the $R^{2}$ values of each were calculated from the experimental data. The maximum value of $R^{2}=0.98$ occurred for $j=1.1$, indicating that in the intermediate temperature range in this system, the spins are mostly uncorrelated, in agreement with mean-field theory. This procedure is shown in Figure 3.7, with the solution for $j=1.1$ displayed as the solid curve in Figure 3.7(a).

In addition, the full-width at half-maximum linewidth $v_{F W H M}^{(1)}$ of the one-magnon resonance was extracted as a function of temperature from the Lorentzian fits. The peaks are expected to be Lorentzian rather than Gaussian because they are caused by relaxation broadening. ${ }^{123}$ The measured values of $v_{F W H M}^{(1)}$ appear to be constant at $0.05 \mathrm{THz}$. However, the linewidth is expected to increase from $0.075 \mathrm{THz}$ to $0.19 \mathrm{THz}$ over the temperature range due to thermal damping of the magnons. ${ }^{118}$ This discrepancy can be attributed to the limited spectral resolution of our method. Further studies can improve upon this result by measuring a longer range of $t$ values. 


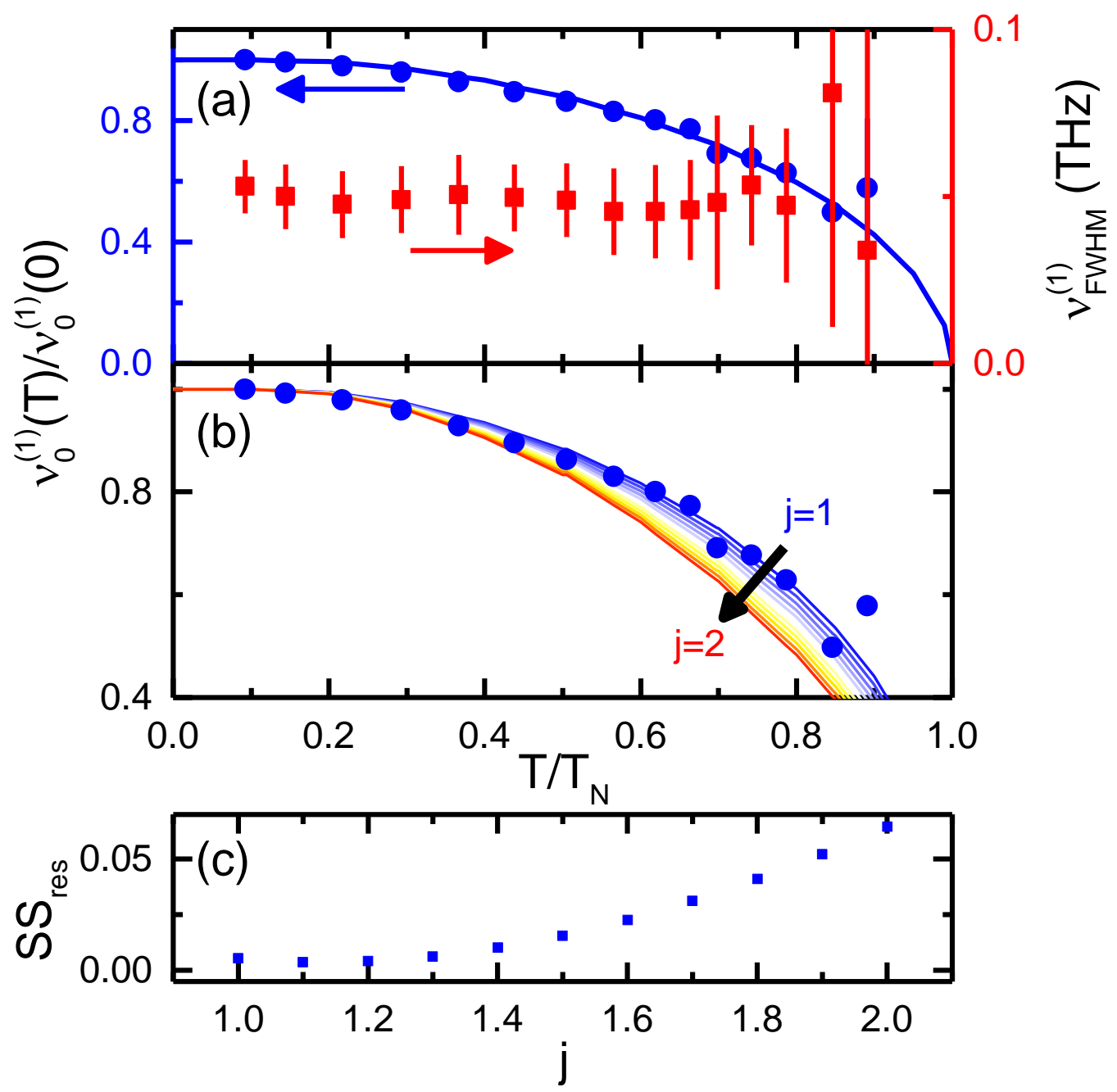

Figure 3.7: (a) Temperature dependence of the normalized center frequency $v_{0}^{(1)}(T) / v_{0}^{(1)}(T)$ (blue circles, left axis), and the FWHM (red squares, right axis) of the one-magnon resonance peak. The solid blue line is a fit using Equation 3.5 with $J=5 / 2$ and $j=1.1$. Standard error from the fitting function is represented by vertical lines. (b) Equation 3.5 for values of $j$ between 1 and 2. (c) Residuals for each $j$ value showing a minimum (and therefore best fit to the data) at $j=1.1$.

We now discuss the high frequency feature in Figure 3.6(a). This feature corresponds to the twomagnon resonance measured previously. ${ }^{116,124}$ The two-magnon peak has a similar amplitude and much larger width than the one-magnon peak, which confirms that the mechanism behind the two resonances must be different. ${ }^{125,126}$ At $T=6.2 \mathrm{~K}$, the maximum occurs at $v_{0}^{(2)}=\sim 1.8 \mathrm{THz}$ with a linewidth of $v_{F W H M}^{(2)}=\sim 1.1 \mathrm{THz}$, and it significantly broadens at temperatures above $T_{N}$. 
One-magnon absorption is primarily produced by absorption of a photon by a Brillouin-zone-center magnon. In contrast, two-magnon absorption is an excited-state exchange interaction in which two zoneedge magnons are produced with opposite momenta $+k$ and $-k \cdot{ }^{127}$ Therefore the magnons in the twomagnon process are distributed over a range of frequencies, which is why a broad peak is produced compared to that of the one-magnon process. In addition, two-magnon processes are known to exist well above the Néel temperature due to persistent short-range magnetic order. ${ }^{119,128}$

\subsection{2: Refractive index}

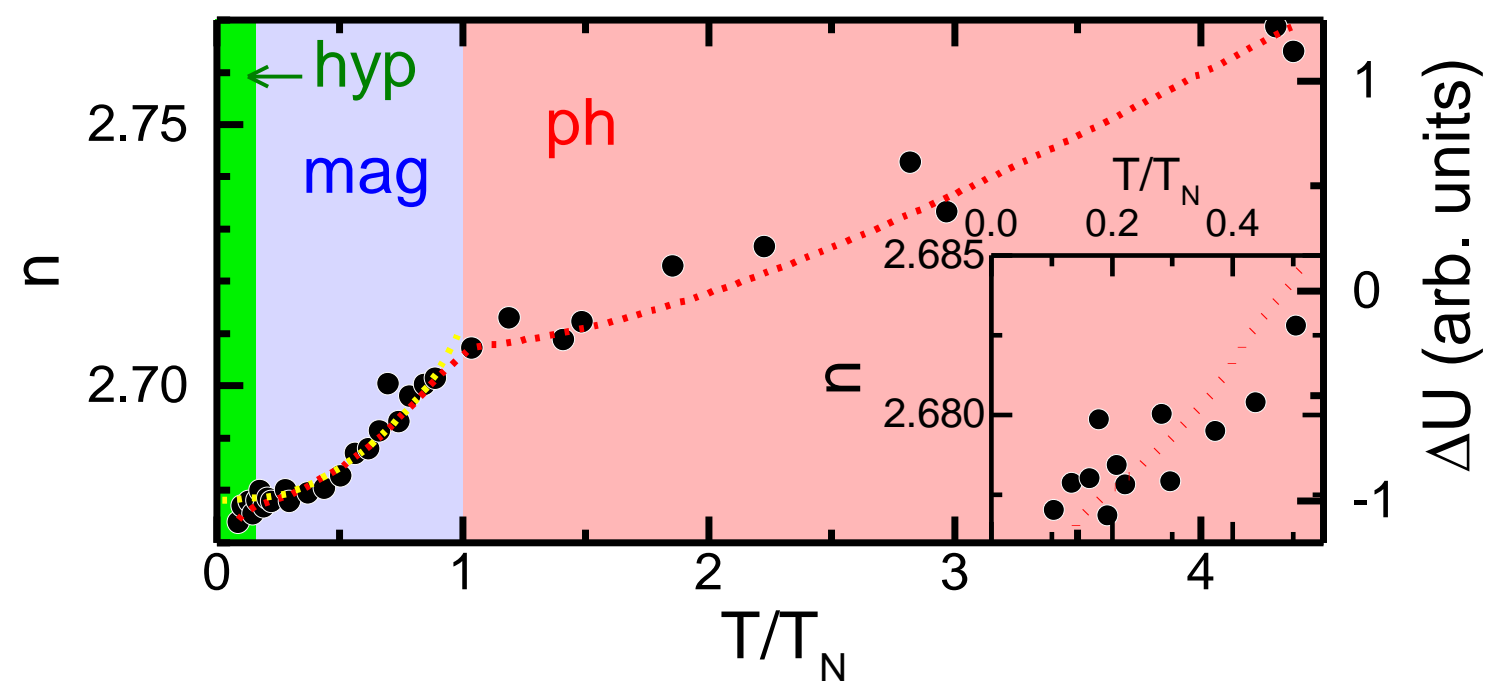

Figure 3.8: Temperature dependence of the $\mathrm{THz}$ refractive index $n$. Three distinct regions are highlighted. In the high temperature region, the behavior is dominated by phonon contributions to the internal energy (ph). In the region $15 \mathrm{~K}<T<T_{N}$, the behavior is primarily determined by magnetism (mag). In the very low temperature region, hyperfine contributions may become significant (hyp). The red curve is a fit to the data using a sum of the three contributions. The yellow curve is the magnetic change in internal energy calculated by Seehra et al. (right axis). ${ }^{129}$ The inset is a zoomed-in view of the low temperature hyperfine region.

Figure 3.5 shows a comparison of the $\mathrm{THz}$ transients, highlighting the difference in delay time caused by a change in refractive index in the optical path. Varying the temperature of the sample changes the arrival time $t_{0}$ of the pulse, as seen by the difference in the green and red curves in the figure.

The temperature dependent $\mathrm{THz}$ refractive index is shown in Figure 3.8. Changes of the refractive index with temperature are related to changes in internal energy $\Delta U$ and Muller's strain-polarizability constant $\Lambda$, such that $\Delta n \propto \Delta U(\Lambda-1) .{ }^{112}$ Given a temperature change from $T_{1}$ to $T_{2}, \Delta U$ is the temperature 
integral of the specific heat at constant volume, $\Delta U=\int_{T_{1}}^{T_{2}} C_{V} d T .{ }^{112}$ The sign of $\Lambda-1$ determines the sign of $\Delta n$, and in the case of $\mathrm{MnF}_{2}$ we see that $\Lambda-1$ is positive. The specific heat is a sum of several terms, $C_{V}=$ $C_{p h}+C_{\text {elec }}+C_{\text {mag }}+C_{\text {hyp }}$, where $C_{p h}$ is the contribution from phonons in the lattice, $C_{\text {elec }}$ is the electronic contribution, $C_{m a g}$ is the magnetic contribution, and $C_{\text {hyp }}$ is the hyperfine contribution. $C_{\text {elec }}$ can be neglected in an insulator like $\mathrm{MnF}_{2}$.

The red curve in Figure 3.8 is a fit of the form

$$
n=n_{0}+A_{p h} \Delta U_{p h}+A_{m a g} \Delta U_{m a g}+A_{h y p} \Delta U_{h y p}
$$

where $n_{0}$ is the offset with no additional contributions, and $A_{p h}, A_{m a g}$, and $A_{\text {hyp }}$ are scaling factors for the three contributions, chosen to fit the data. The value of $n_{0}$ was determined by this fit to be $\sim 2.71$, indicating that this would be the $\mathrm{THz}$ refractive index if one could turn off phonons, magnons, and hyperfine interactions in the system. The presence of phonons adds energy to the system, so $\Delta U_{p h}$ is positive, resulting in a positive change in $n$. The addition of magnetic order decreases the energy of the system, so $\Delta U_{\text {mag }}$ is negative, resulting in a negative change in $n$. The presence of the hyperfine field at very low temperature also decreases the energy of the system, so $\Delta U_{h y p}$ is negative as well.

Above $T_{N}$ (red region in the figure), the only significant contribution to the internal energy is phonons. The temperature dependence of the phonon contribution comes from the Debye model and can be written as

$$
\Delta U_{p h}=9 N k_{B} T\left(\frac{T}{\theta_{D}}\right)^{3} \int_{0}^{x_{D}} d x \frac{x^{3}}{e^{x}-1}
$$

where $x_{D}=\theta_{D} / T, N$ is the number of atoms in the sample, $k_{B}$ is Boltzmann's constant, and $\theta_{D}$ is the Debye temperature, $381 \mathrm{~K}$ for $\mathrm{MnF}_{2 .}{ }^{130}$ The data in the thermal expansion region near $T / T_{N}$ deviate slightly above the fit, which may be explained by persistent short-range magnetic order.

Deviation from the thermal expansion model below $T_{N}$ is due to the magnetic internal energy (blue region). $\Delta U_{\text {mag }} \propto-\left(M / M_{0}\right)^{2}$ in a simple picture excluding next-nearest neighbor and next-next-nearest neighbor contributions. The temperature dependence of the normalized magnetization $M / M_{0}$ is described 
in Section 3.3.1, and here the same model is used with the scaling factor $A_{m a g}$ to fit the data. For comparison, we show the magnetic internal energy calculated by Seehra et al. from experimental magnetic specific heat data ${ }^{129}$ as the yellow dotted curve (right axis). In the region $15 \mathrm{~K}<T<T_{N}$, our fit closely follows this calculation. Above $T_{N}$, some remnant magnetization remains which may contribute to the change in refractive index. Below $15 \mathrm{~K}$, the calculation diverges from our measured data.

Including only $\Delta U_{p h}$ and $\Delta U_{m a g}$ describes the data very well, resulting in an $R^{2}$ value of 0.92 . However, we attempt to go one step further to explain the low temperature deviation from this model. To this end, we include the hyperfine contribution to the internal energy, which is described by $\Delta U_{h y p} \propto-1 / T$. In fluorine, hyperfine effects have been observed at $12 \mathrm{~K}$ and below. ${ }^{131}$ Hyperfine contribution to the specific heat can be observed at low temperature in other Mn compounds as well, ${ }^{132,133}$ owing to the high spin (5/2) of the Mn nucleus. Including the hyperfine contribution is only a minor improvement to the overall fit, increasing the $R^{2}$ value by less than 0.01 . The data points in the region $T \leq 15 \mathrm{~K}$ are averaged over multiple independent data sets, providing high confidence that the true values deviate below the magnetic fit at low temperatures.

\section{4: Conclusions}

The all-optical technique of $\mathrm{THz}$ spectroscopy was used to examine the prototypical AF material, $\mathrm{MnF}_{2}$. The one- and two-magnon resonances were observed by the relative change in transmission at the resonant frequencies, and studied as a function of temperature. The one-magnon resonance was shown to redshift at higher frequencies up to the Néel temperature, above which there is no longer any coherence, and the curve was matched to molecular field theory. Additionally, our fit of the resonance frequency suggests that neighboring spins are predominantly uncorrelated in the temperature range $20 \mathrm{~K}<T<50 \mathrm{~K}$.

The excited-state exchange two-magnon peak was shown to persist above the Néel temperature and broaden at high temperatures. In addition, the index of refraction was measured by determining the speed of the $\mathrm{THz}$ pulse passing through the sample by exploiting the transient nature of the experiment.

The temperature dependence of the index of refraction between $6 \mathrm{~K}$ and room temperature was 
successfully modeled by taking into account contributions from phonons, magnetic ordering, and hyperfine interactions.

The techniques shown here can be generalized to any spin-coherent sample with a resonance in the available frequency range, which can be improved further by careful choice of cryostat windows, detection crystal, and $\mathrm{THz}$ source. Using this technique, new AF materials may be made available and thoroughly characterized for their potential in spintronic applications. 


\section{Chapter 4: All-Optical Injection of Photocurrents into}

\section{$\mathrm{Bi}_{2} \mathrm{Se}_{3}$}

In the context of spintronics, this chapter attempts to further the understanding of carrier dynamics in TIs. Bulk charge carriers in an ideal TI are tightly bound as in an insulator. However, because of the topology discussed in Section 1.3, surface charge carriers are inextricably linked to their spin states, making them ideal channels for both spin currents and spin-polarized charge currents. These bulk and surface energy bands have been directly observed with ARPES ${ }^{134}$ but there is much to learn about the degree of spin-polarization, the relative contributions of the various transitions bulk-bulk, bulk-surface, surface-bulk, and surface to surface, and the effect of TI film thickness on carrier dynamics. Although $\mathrm{Bi}_{2} \mathrm{Se}_{3}$ is often considered to be the prototypical $\mathrm{TI}$, the fact that its bulk is naturally $\mathrm{n}$-doped in as-grown samples is problematic because any currents observed from $\mathrm{Bi}_{2} \mathrm{Se}_{3}$ samples could conceivably be coming from a combination of bulk and surface electrons. Nonetheless, in this work we set out to all-optically inject currents into $\mathrm{Bi}_{2} \mathrm{Se}_{3}$ samples and follow this up with a thorough investigation of an analysis of parameter space in order to determine the extent to which we are sensitive to the interesting topological SS.

This chapter discusses all-optical measurements of excited carrier dynamics in films of the TI $\mathrm{Bi}_{2} \mathrm{Se}_{3}$, grown by molecular beam epitaxy with in-situ reflection high-energy electron diffraction. The films are shown to be high-quality by x-ray reflectivity and diffraction and atomic-force microscopy. Quantum interference control of photocurrents is observed by excitation with harmonically related pulses and detected by $\mathrm{THz}$ radiation. The IC obeys the expected excitation irradiance dependence, showing linear dependence on the fundamental pulse irradiance and square-root irradiance dependence of the frequencydoubled optical pulses. The IC also follows a sinusoidal relative-phase dependence between the two excitation pulses. These results confirm the third-order nonlinear optical origins of the coherently controlled IC. Experiments are compared to a tight-binding band structure to illustrate the possible optical transitions that occur in creating the IC. 


\section{1: $\mathrm{Bi}_{2} \mathrm{Se}_{3}$ thin-film growth by molecular beam epitaxy}

$\mathrm{Bi}_{2} \mathrm{Se}_{3}$ films of various thickness were grown by molecular beam epitaxy ${ }^{135}$ on double-side polished $0.5-\mathrm{mm} \mathrm{Al}_{2} \mathrm{O}_{3}(0001)$ substrates by a two-step process. ${ }^{136}$ In the first step, three QLs were grown at $140{ }^{\circ} \mathrm{C}$ to help deposition of the film with the correct stoichiometry. The remainder of the film is grown in a second step at a temperature of $275^{\circ} \mathrm{C}$ to produce a smoother film. Figure 4.1 (a) shows the oscillations of layer growth during the high-temperature growth stage, monitored by the in-situ reflection high-energy electron diffraction (RHEED). The inset of the figure shows the striping associated with a high-quality film. RHEED oscillations confirm growth of the 12 additional QLs of $\mathrm{Bi}_{2} \mathrm{Se}_{3}$ to form a $15-\mathrm{QL}$ thick sample.

The primary experiments were all performed on this 15-QL sample, but other samples of thickness $6,8,10,12,20,25,30,35$, and 40 QL were all grown using the same method, and these were used in thickness-dependence measurements discussed later on.

It is suspected that Se vacancies ${ }^{14,137}$ quickly alter the surface potential and subsequently lead to oxidation when exposed to air. Consequently, a $10-\mathrm{nm}$ thick $\mathrm{MgF}_{2}$ capping layer is sputtered onto the samples without removing them from the growth chamber. The result is an encapsulated and highly stable film of $\mathrm{Bi}_{2} \mathrm{Se}_{3}$.

$\mathrm{Bi}_{2} \mathrm{Se}_{3}$ film thicknesses were then confirmed with x-ray reflectivity (XRR); see Figure 4.1(b). Fitting the XRR pattern indicates roughness of $0.2 \mathrm{~nm}$. Fitting of the XRR data is performed using the open-source program GenX. The inset shows an atomic force micrograph of the $\mathrm{Bi}_{2} \mathrm{Se}_{3}$ film with $\mathrm{MgF}_{2}$ layer. The triangles reveal the expected degree of dislocations in the $\mathrm{Bi}_{2} \mathrm{Se}_{3}$ film. Figure 4.1(c) shows x-ray diffraction (XRD), which is used to determine the degree of the out-of-plane disorder. The 003 XRD Bragg peak observed at low angle corresponds to that observed in the XRR data. Overall, characterization indicates high-quality films with which to perform optical measurements. 

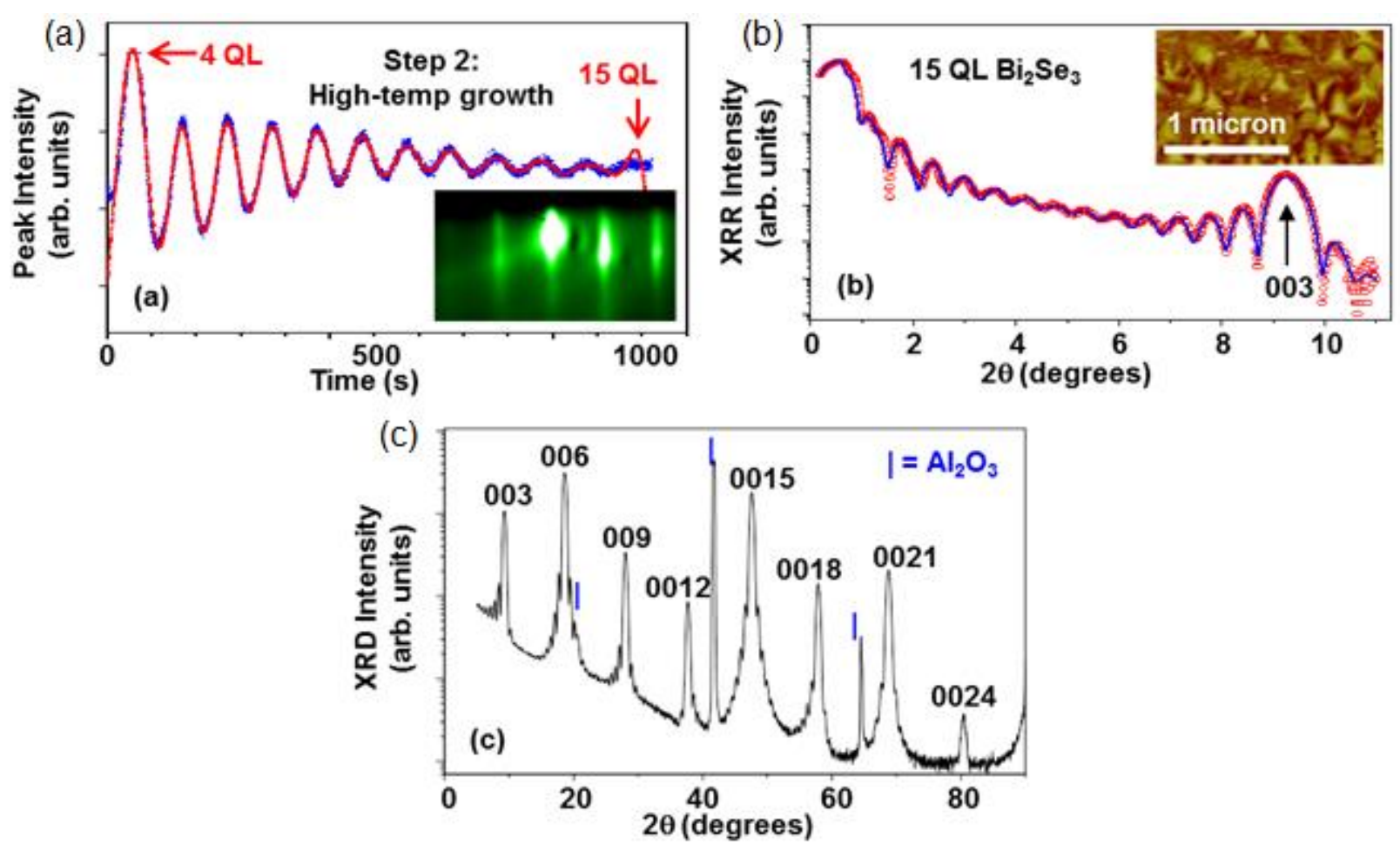

Figure 4.1: (a) Reflection high-energy electron diffraction during the second growth stage of the $\mathrm{Bi}_{2} \mathrm{Se}_{3}$ film. The oscillations indicate growth of $12 \mathrm{QLs}$ in the formation of a 15-QL sample. $1 \mathrm{QL}$ is approximately $1 \mathrm{~nm}$. The inset shows clean stripes indicating good sample quality. (b) X-ray reflection of the 15-QL sample. The inset shows an atomic force micrograph of the sample. (c) Outof-plane x-ray diffraction showing features from the substrate and $\mathrm{Bi}_{2} \mathrm{Se}_{3}$ film.

\section{2: Injection of two-color photocurrents}

Figure 4.2 shows the experimental setup for the current injection measurements along with a schematic of the sample structure. A laser amplifier system with OPA provides $\sim 80$-fs pulses at a repletion rate of $1 \mathrm{kHz}$. Signal pulses from the OPA are centered at $1540 \mathrm{~nm}(0.8 \mathrm{eV})$. A $\beta$-barium borate crystal frequency doubles the fundamental pulses, generating second-harmonic pulses at $770 \mathrm{~nm}(1.6 \mathrm{eV})$. These fundamental $(\omega)$ and frequency-doubled $(2 \omega)$ pulses feed a two-color Mach-Zehnder interferometer that allows for independent control of the phase and polarization for each color. The two colors are set to collinear polarization and their time delay is adjusted to be close to the center of their envelopes, as measured with an interferometric cross-correlation. Measurements presented here are performed on the 15-QL thick $\mathrm{Bi}_{2} \mathrm{Se}_{3}$ sample at normal incidence. Measurements have also been performed on samples ranging from $6 \mathrm{QL}$ to $40 \mathrm{QL}$ showing qualitatively consistent behavior. 


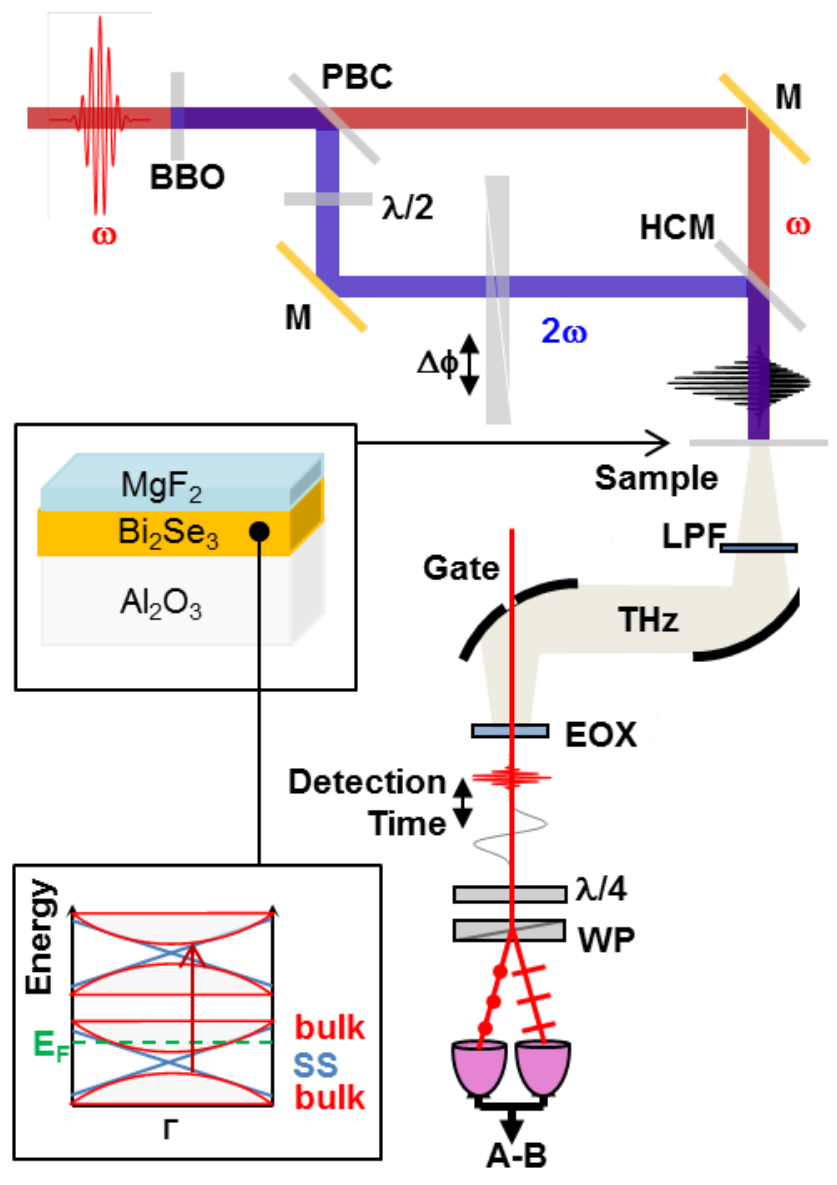

Figure 4.2: Experimental setup of the all-optical IC excitation and detection scheme. $\mathrm{BBO}=$ beta-barium borate crystal, $\mathrm{PBC}=$ polarization beam cube, $\lambda / 2=$ half-wave plate, $\mathrm{M}=$ mirror, $\Delta \phi=$ relative phase between fundamental $(\omega)$, second-harmonic $(2 \omega)$ pulses, $\mathrm{HCM}=$ hot-cold mirror, LPF $=$ low-pass filter, EOX = electro-optic sampling crystal, $\lambda / 4=$ quarter-wave plate, $\mathrm{WP}=$ Wollaston prism, $A-B=$ balanced detection signal feed to lock-in amplifier. The top inset shows the structure of the sample. The lower inset shows the band structure for a slab of $\mathrm{Bi}_{2} \mathrm{Se}_{3}$.

The acceleration of charge in the production of the photocurrent on a picosecond timescale reradiates light at $\mathrm{THz}$ frequencies. ${ }^{44,138}$ The sample is oriented such that the optical pulses transmit through the sapphire substrate before they arrive at the $\mathrm{Bi}_{2} \mathrm{Se}_{3}$ film, so as to not distort the emitted $\mathrm{THz}$ radiation. ${ }^{139}$ The $\mathrm{THz}$ radiation is collected using off-axis parabolic mirrors and focused onto a 1-mm thick EO crystal (ZnTe), which is gated by an 80 -fs pulse from the laser amplifier. The quasi-static electric field of the $\mathrm{THz}$ induces birefringence in the $\mathrm{ZnTe}$ that is seen by the gate pulse and measured by projecting the orthogonal polarization components onto balanced photodetectors. ${ }^{75}$ The signal is extracted using lock-in detection referenced to a mechanical chopper placed close to the laser source, which modulates the light at $250 \mathrm{~Hz}$. 
The $\mathrm{THz}$ signal is mapped out in the time domain by varying the relative time delay between the emitted $\mathrm{THz}$ and the gate pulse.
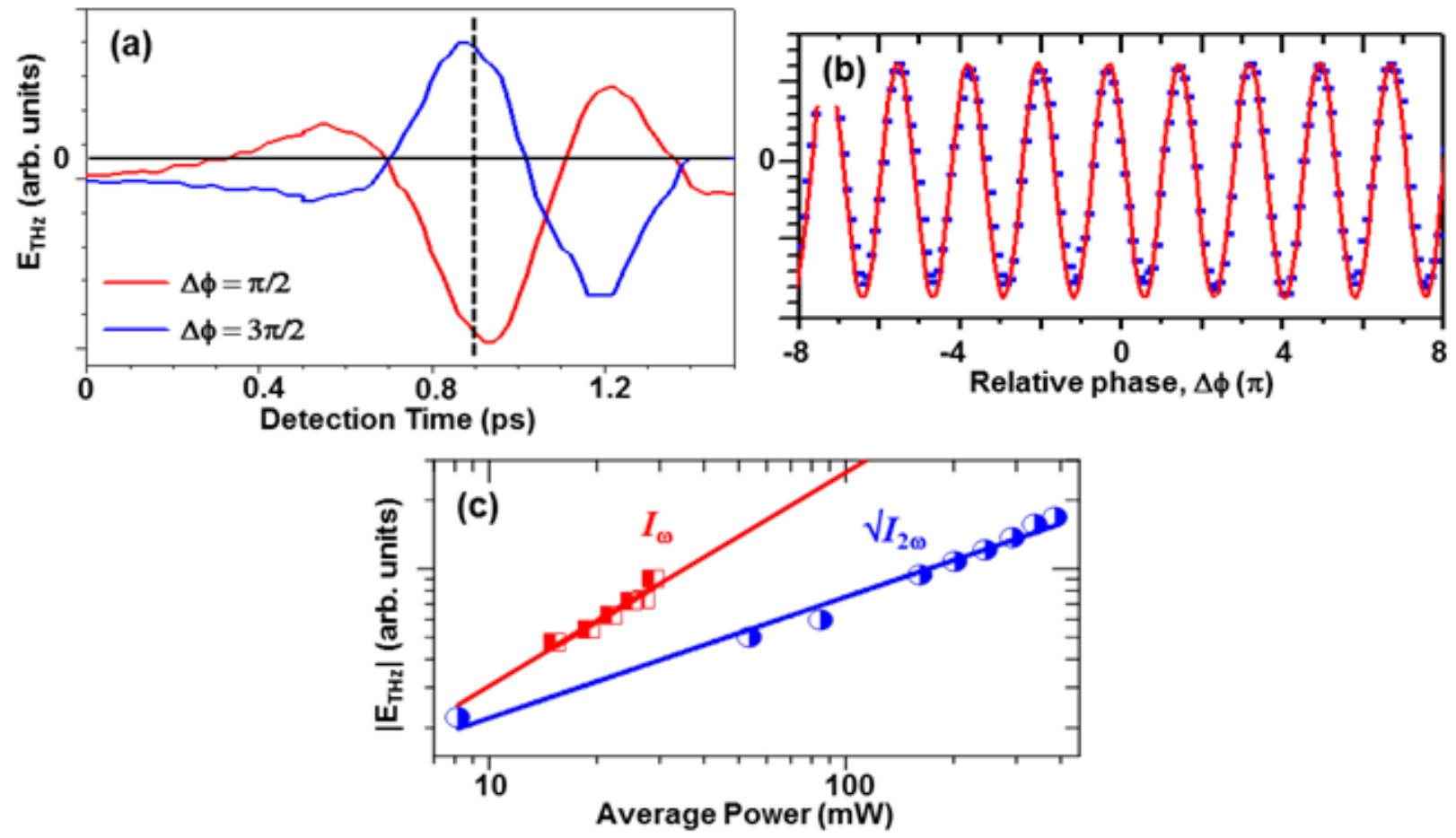

Figure 4.3: (a) $\mathrm{THz}$ transients recorded with relative phase conditions between the $\omega$ and $2 \omega$ excitation pulses of $\pi / 2$ and $3 \pi / 2$. (b) Phase dependence recorded at the peak of the $\mathrm{THz}$ transient indicated in (a). (c) Average power (or irradiance) dependence of the $\omega$ and $2 \omega$ excitation pulses individually, while fixing the average power (or irradiance) of the other.

Figure 4.3(a) shows $\mathrm{THz}$ transients recorded for two excitation conditions of the relative phase $\left(\Delta \phi=2 \phi_{\omega}-\phi_{2 \omega}\right)$ between the $\omega$ and $2 \omega$ optical pulses. It can be seen that for an excitation condition of $\Delta \phi=3 \pi / 2$ the transient has the opposite sign to that for $\Delta \phi=\pi / 2$. Figure $4.3(\mathrm{~b})$ shows a sinusoidal dependence of the $\mathrm{THz}$ amplitude versus $\Delta \phi$, measured at the peak of the $\mathrm{THz}$ transient indicated by the dashed vertical line in Figure 4.3(a). In Figure 4.3(b) a slight offset from zero is observed indicating that there exists a weak background that is phase independent and results from excitation due to only one pulse.

Figure 4.3(c) shows the dependence of the emitted THz amplitude on irradiance (or average power) of one excitation pulse while holding the other constant and vice versa. Fixing the average power of the $\omega$ pulse and varying the average power of the $2 \omega$ pulse results in a sub-linear slope on the log-log plot, with 
a $\sqrt{I_{2 \omega}}$ dependence. In this case, the THz amplitude scales because of 1PA of the $2 \omega$ pulse. In contrast, fixing the average power of the $2 \omega$ pulse and varying the average power of the $\omega$ pulse results in a linear slope, with an $I_{\omega}$ dependence. In this case, the THz amplitude scales due to two-photon absorption (2PA) of the $\omega$ pulse. Overall, the emitted field amplitude of the $\mathrm{THz}$ signal follows a $\sqrt{I^{3}}$ (or $I^{1.5}$ ) dependence, confirming that it is governed by a third-order nonlinear optical process.

The relative-phase dependence and average-power dependence of the emitted $\mathrm{THz}$ signals are the expected signatures of a signal derived from an IC. ${ }^{51}$ ICs arise from an imbalance of the total number of carriers excited to positive and negative states in k-space. The net momentum is equivalent to excitation of a ballistic photocurrent. In this all-optical method of injecting charge currents, the emitted $\mathrm{THz}$ follows the derivative of the IC $J_{i}$, such that $\frac{d J_{i}}{d t}=2 \eta_{i j k l}(0 ; \omega, \omega,-2 \omega)\left|E_{j k}^{\omega}\right|^{2} E_{l}^{2 \omega} \sin \Delta \phi$, where $\eta_{i j k l}$ is a fourth-rank tensor related to the imaginary part of $\chi^{(3)}, E_{j k}^{\omega}$ is the $\omega$ electric field, $E_{l}^{2 \omega}$ is the $2 \omega$ electric field, and $i, j, k$, and $l$ are numerical indices. ${ }^{140}$ Using the above equation, the relative-phase dependence and average powerdependences have been fit, shown as solid lines in Figure 4.3(b) and (c) respectively. Overall, the theoretical comparison qualitatively fits the experimental data well. At the time of this work, quantitative analysis could not be performed because little was known about the nonlinear absorption coefficients for this material due to SA. ${ }^{19}$ Nonetheless, theoretical verification of the irradiance and relative phase dependence exclude other $\mathrm{THz}$ generation processes such as $\mathrm{OR}^{75}$ and SCs. ${ }^{44}$

Third-order nonlinear processes are expected from all materials and ICs have been observed in a range of solids, including unbiased and unstrained silicon, ${ }^{50}$ direct-gap GaAs, ${ }^{138}$ nanowires, ${ }^{141}$ and graphene. ${ }^{142}$ The process in $\mathrm{Bi}_{2} \mathrm{Se}_{3}$ may be similar to that in graphene if Dirac SS are involved. In which case, quantum interference between single and 2PA at $2 \omega$ occurs in the presence of simultaneous 1PA of the $\omega$ pulse. In graphene, the absorption at $\omega$ interferes with the efficiency of the IC, ${ }^{62}$ so we expect it may in the case of $\mathrm{Bi}_{2} \mathrm{Se}_{3}$ as well.

The inset of Figure 4.2 shows a band structure for a $15-\mathrm{QL} \mathrm{Bi}_{2} \mathrm{Se}_{3}$ thin film calculated using a phenomenological tight-binding model, based on eight bands to capture both sets of SS and Dirac cones. The parameters are optimized for a bulk system using results from local-density approximation 
calculations $^{134,143}$ and two-photon photoemission measurements. ${ }^{40}$ Confinement-induced bands are determined by solving the model in the slab geometry. The $2 \omega$ frequency is also illustrated and is commensurate with excitations from the SS near the $\Gamma$-point of the band structure below the Fermi surface to states in the newly discovered set of SS at close to $1.7 \mathrm{eV}$.

At the wavelength of excitation, transitions depend on the character of the bulk and SS. There are a variety of transitions that include surface, bulk, and both types of states. Indeed, the surface-surface transitions and bulk-bulk transitions should have very different characteristics. Moreover, bulk-surface or surface-bulk transitions lead to a charge displacement in the thin films. Finally, in thin films confinement can lead to new bands in the band structure drastically changing the ratio of surface and bulk states suitable for optical transitions. ${ }^{11}$

\section{3: Outlook}

$\mathrm{Bi}_{2} \mathrm{Se}_{3}$ films have been grown to sufficient quality that ICs have been observed and controlled using all-optical methods. All-optical methods of charge current excitation and detection are non-invasive and avoid problems with contacts, such as local modification of band structures and slow read-outs that can confuse current signals. The ability to inject and control these photocurrents offers a powerful method for characterization of $\mathrm{TI}$ materials based on as-grown $\mathrm{Bi}_{2} \mathrm{Se}_{3}$.

The ultrafast coherent control of ballistic currents has been demonstrated in varying thicknesses of thin-film $\mathrm{Bi}_{2} \mathrm{Se}_{3}$. Currents were injected and detected through all-optical methods and were demonstrated to show the expected signatures for a $\chi^{(3)}$ current injection process as has been demonstrated previously in prototypical semiconductors. Such a control scheme is a valuable tool for the continued study of ultrafast carrier dynamics in the nontrivial TI SS. 


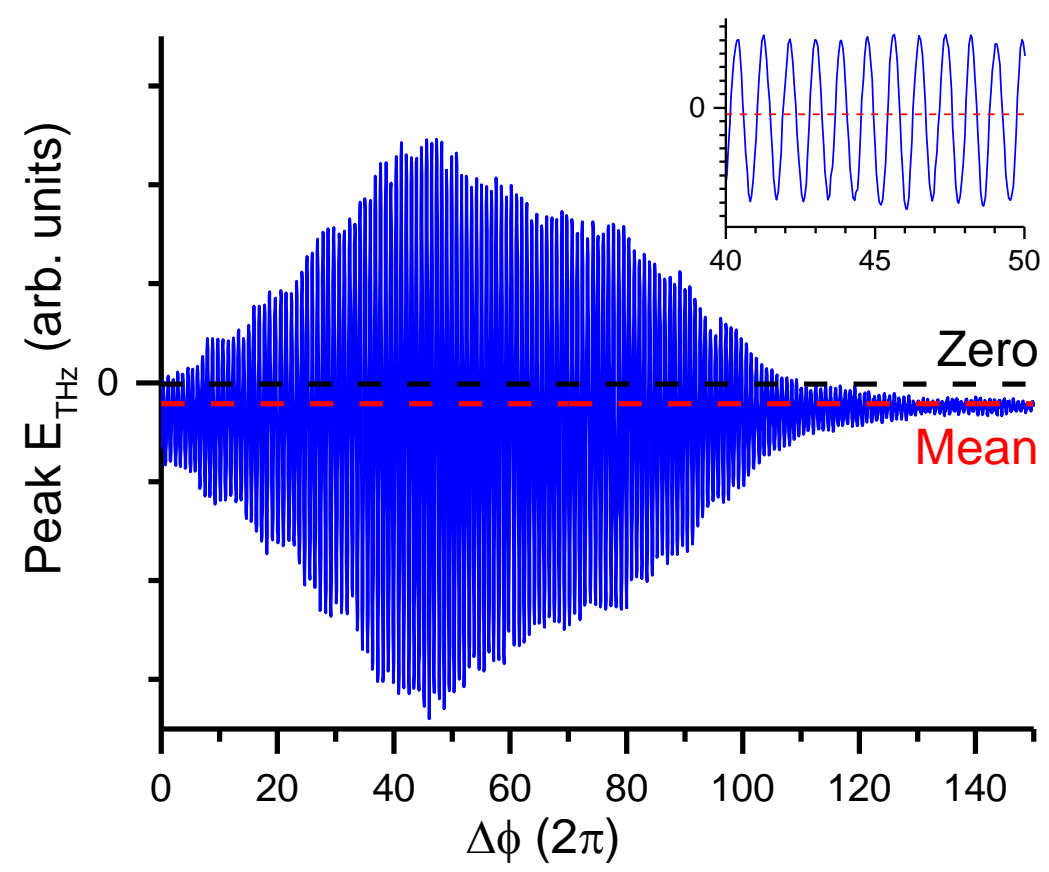

Figure 4.4: Relative phase dependence of the IC over the entire envelope of the pump pulses. Inset is a zoomed-in portion with the same axes. The sinusoidal dependence of the IC is contained in a Gaussian envelope. The mean ( $\Delta \phi$ - independent) signal is nonzero, indicating that the measured signal includes more contributions than the simple IC alone.

Although the expected signatures of IC are readily apparent, we see additional behavior such as that in Figure 4.4. With only a single pump beam present, the IC is expected to be zero. Because of symmetry, the average photocurrent when varying over a long range of $\Delta \phi$ values should be zero. However, we see a clear offset in our data. Therefore, additional photocurrent contributions to the signal must be explored. Chapter 5 will explore this in further detail.

\subsection{1: Thickness dependence}

By measuring an entire $\mathrm{THz}$ trace over several picoseconds and recording how it changes with relative phase we obtain graphs such as that shown in Figure 4.5. Here, the qualitative behavior is shown for the 15-QL sample, but the behavior of all samples is similar. The characteristic checkerboard pattern has been observed in IC in other types of samples as well, such as GaAs and silicon. In Chapter 5 we discuss the properties of these graphs and deviations from it due to sample symmetries. 


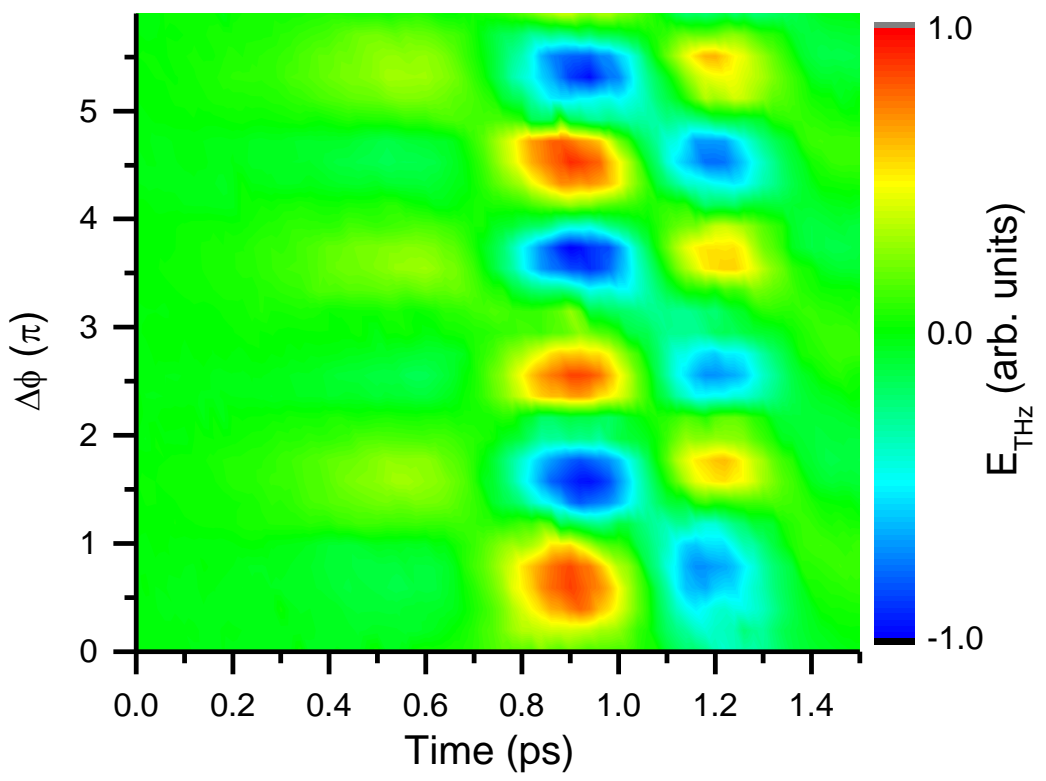

Figure 4.5: Dependence of the IC on time and relative phase. Graphs for each sample thickness are qualitatively similar but differ in magnitude.

Combined with other studies, the unique and interesting signatures of SS can be directly observed through thorough thickness- and photon energy- dependence measurements. These methods are an important next step towards the eventual use of Tls in novel electronic and spintronic devices. Quantum interference control is a valuable research tool because it gives ultrafast control over not only power (as was demonstrated in this work), but polarization and photon energy as well. Future work will be dedicated to accessing this further control and unlocking the previously inaccessible features of TI SS spin-locking properties and dynamics.

\subsection{2: Photon energy dependence}

In addition to thickness, it is important to examine the effect of exciting electrons to various energy levels surrounding the Dirac cone. Figure 4.6 is a schematic illustrating the available range of photon energies in the current experimental setup. Here a BBO crystal is used to double any given frequency and extend the available range. A second BBO could be used to extend the range even further, but this would result in inefficient conversion and a weak pump beam. 


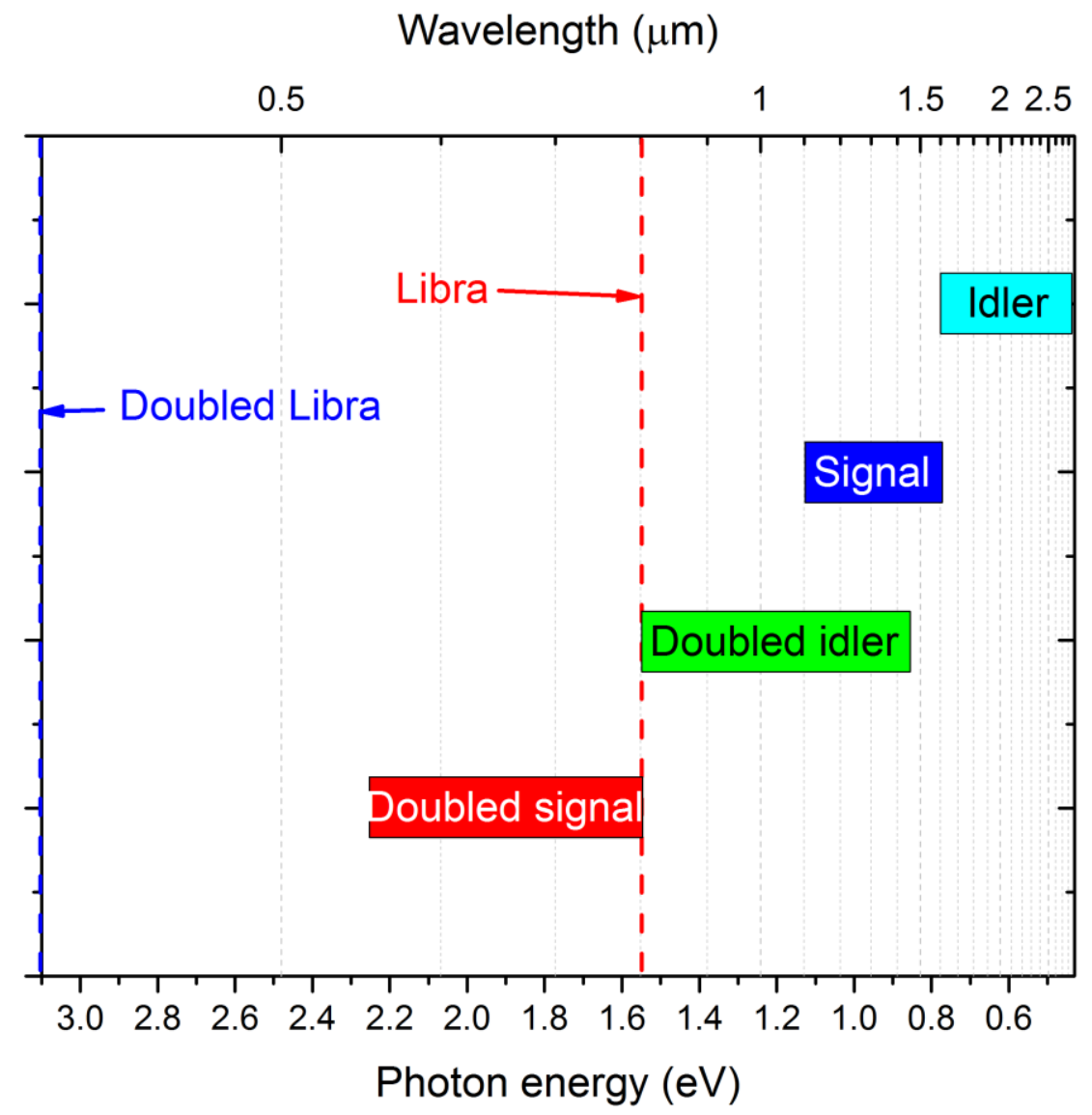

Figure 4.6: Photon energies available with the experimental setup.

The vertical lines labelled "Libra" and "Doubled Libra" are the most efficient pump energies as they are achieved using the beam straight out of the laser amplifier. The sum-frequency beam tuned by the OPA is labelled "Signal" or "Idler" depending on the configuration of the OPA, allowing us to reach 1200 to $2600 \mathrm{~nm}$. By doubling the OPA output we can decrease the wavelength to the range 600 to $1300 \mathrm{~nm}$. Chapter 6 will examine how this range of photon energies can be related to the band structure of $\mathrm{Bi}_{2} \mathrm{Se}_{3}$. 


\section{Chapter 5: Separation of Photocurrents in $\mathrm{Bi}_{2} \mathrm{Se}_{3}$}

Optical injection and detection of charge currents can complement conventional transport and photoemission measurements without the necessity of invasive contact that may disturb the system being examined. This is a particular concern for the SS of a TI. In this chapter, one- and two-color sources of photocurrents are examined in epitaxial, thin films of $\mathrm{Bi}_{2} \mathrm{Se}_{3}$. We demonstrate that optical excitation and THz detection simultaneously captures one- and two-color photocurrent contributions, as previously unseen in other material systems. A method is devised to isolate the two components, and in doing so each can be related to surface or bulk excitations through symmetry. This strategy allows SS to be examined in a model system, where they have independently been verified with angle-resolved photoemission spectroscopy.

\section{1: Experiment}

Details of sample growth can be found in D. A. Bas et al. ${ }^{64}$ and P. Tabor et al. ${ }^{135}$ In Figure 5.1(a) we show an experimental sample consisting of three distinct materials: A 500- $\mu \mathrm{m}$ sapphire substrate which is transparent to the pump radiation; a 12-nm TI grown via molecular beam epitaxy; and a $10-\mathrm{nm} \mathrm{MgF}_{2}$ capping layer added to prevent exposure to atmosphere and inhibit the build-up of space charge fields caused by band bending near the surface..$^{41,42}$ In the $\mathrm{TI}$, bismuth (Bi) and selenium (Se) naturally grow in layers as shown in Figure 5.1(b), which shows four rhombohedral unit cells. Bi layers are always surrounded by Se layers, but Se layers take two unique positions: Se2 layers are surrounded by Bi, but Se1 layers are separated by van der Waals bonds on one side with other Se1 layers. This van der Waals interface occurs every five atomic layers, and causes samples to naturally grow in whole number QL intervals, with an Se1 layer on the end. In $\mathrm{Bi}_{2} \mathrm{Se}_{3}, 1 \mathrm{QL}$ is approximately $1 \mathrm{~nm}$ thick. The layered structure makes the material applicable for exfoliation, which has been used in many other studies. 


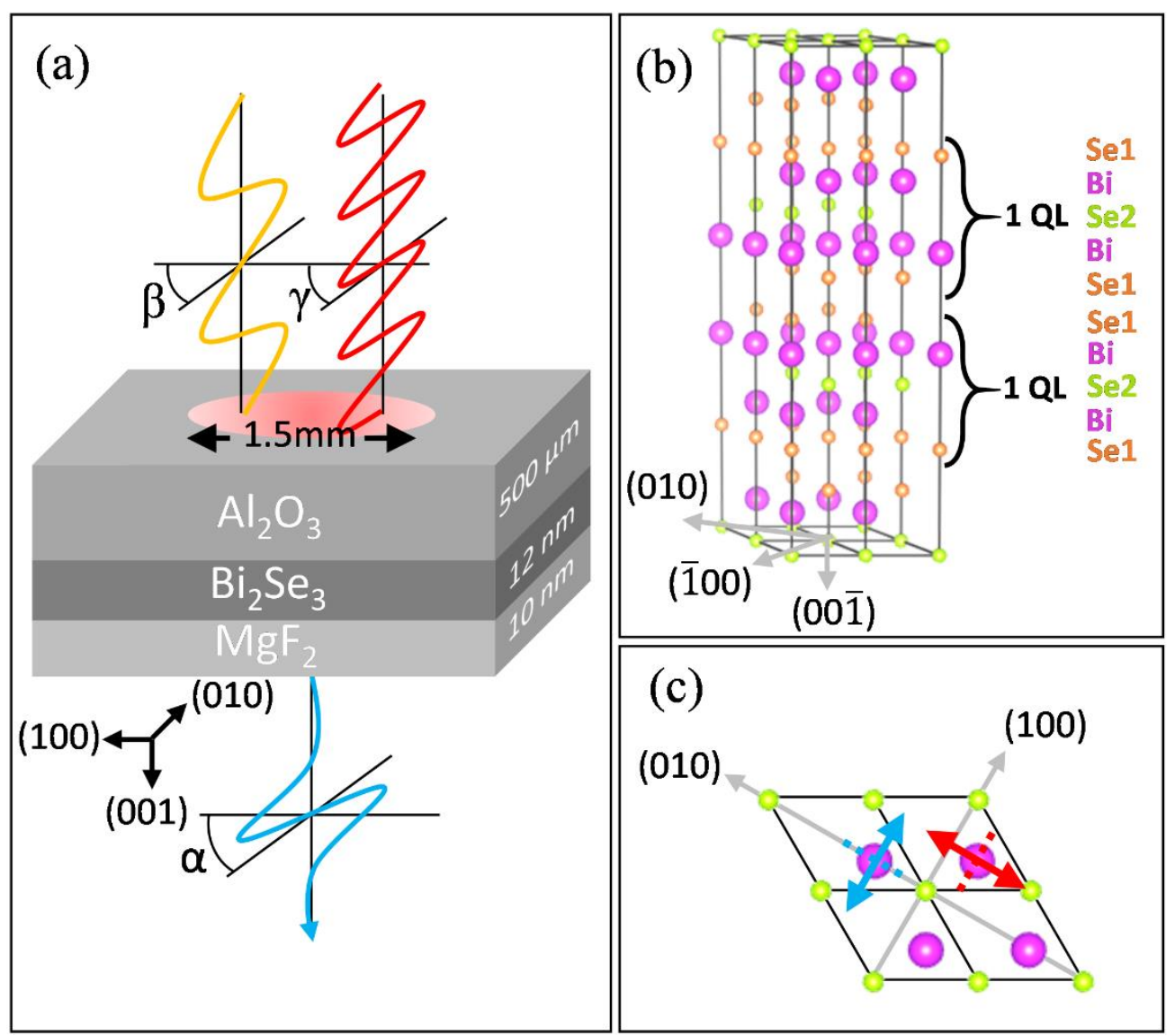

Figure 5.1: Schematic diagram of experimental configuration. (a) Here $\beta$ and $\gamma$ are the angles that the $\omega$ and $2 \omega$ pump polarizations make with the (100) axis of the TI; in the experiments reported here $\beta=\gamma$. The angle of the polarization of the detected $\mathrm{THz}$ relative to the (100) axis of the $\mathrm{TI}$ is denoted by $\alpha$; in this work $\alpha=\beta$ corresponds to the polarization of the detected $\mathrm{THz}$ parallel to the polarization of the incident fields, and $\alpha=\beta+\pi / 2$ corresponds to the polarization of the detected $\mathrm{THz}$ perpendicular to the polarization of the incident fields. (b) Four unit cells, showing the trigonal symmetry of the $\mathrm{Bi}_{2} \mathrm{Se}_{3}$ lattice. (c) Top-down view of the top two atomic layers shown in (b), with example pump polarizations. The blue is a symmetry plane for which the lattice looks identical in both directions. For red, the symmetry is broken and a directional SC can occur. For $\alpha=\beta+\pi / 2$, the relevant symmetry plane is along the direction of the polarization vector.

The experimental setup ${ }^{64}$ uses a laser amplifier system with an OPA to provide $\sim 80$-fs pulses at a repletion rate of $1 \mathrm{kHz}$. The signal pulse from the OPA centered at $1540 \mathrm{~nm}(0.8 \mathrm{eV})$ pumps a $\beta$-barium borate crystal to generate second-harmonic pulses at $770 \mathrm{~nm}(1.6 \mathrm{eV})$. These fundamental $(\omega)$ and frequency-doubled $(2 \omega)$ pulses feed a two-color Mach-Zehnder interferometer that independently controls the intensity, phase, and polarization of the two pulses. The combined pulses impinge at normal incidence the $\mathrm{Bi}_{2} \mathrm{Se}_{3}$ sample which sits in a rotation mount. The acceleration of charge due to the production and decay of the photocurrent leads to $\mathrm{THz}$ radiation, which is collected using off-axis parabolic mirrors and 
detected with EO sampling. The 80 -fs gate pulses used for EO sampling are derived from the laser amplifier. By varying the delay time $t_{\text {det }}$ of the gate pulse the $\mathrm{THz}$ signal is mapped out in the time domain. The time of maximum field detected resulting from the $\omega$ pump alone is indicated by $t_{d e t}=0$. The detection is set up to measure the components of the $\mathrm{THz}$ radiation polarized parallel and perpendicular to the polarization of the incident radiation.

The powers of the pulse trains at $\omega$ and $2 \omega$ were approximately $35 \mathrm{~mW}$ and $1 \mathrm{~mW}$, respectively. Both beam diameters were collimated to have a $1 / e^{2}$ diameter of approximately $1.5 \mathrm{~mm}$, and were colinearly polarized in a fixed direction with the azimuthal rotation of the sample controlled by a rotation mount. Figure 5.1(a) illustrates a generalized case where $\beta$ and $\gamma$ are the angles that the $\omega$ and $2 \omega$ pumps make with the (100) axis of the $\mathrm{TI}$, but throughout this work $\beta=\gamma$. When the (100) axis of the $\mathrm{TI}$ is parallel with the pump polarization, $\beta$ is set to zero. Lattice orientation for the sample was confirmed by $\mathrm{x}$-ray diffraction measurements; however, a distinction is not made between (100) and (100) directions. We use $\alpha$ to denote the polarization of the measured THz field, and for this work orthogonal components at $\alpha=\beta$ (parallel) and $\alpha=\beta+\pi / 2$ (perpendicular) were recorded. Detection was calibrated using a strong OR source. ${ }^{75}$ The $\omega$ and $2 \omega$ pulses transmitted first through the substrate and then the $\mathrm{TI}$ to avoid stretching of the $\mathrm{THz}$ emission by the substrate. ${ }^{139}$ Optical phase walk-off of the pump pulses due to misalignment was corrected by adjusting the two-color interferometer.

The path length of the $\omega$ pulse was fixed throughout the entire experiment. The delay time $\tau$ of the $2 \omega$ pulse (and hence the phase parameter $\Delta \phi=\phi_{2 \omega}-2 \phi_{\omega}$ ) was controlled by a pair of glass wedges and $\tau=0$ was identified with the point of maximum IC within the cross-correlation of the two excitation pulses. By adjusting $\tau$ beyond the cross-correlation envelope, one might expect the IC to be completely suppressed, allowing the possibility of independently measuring a SC and comparing it to the IC. However, it has been shown before, ${ }^{144}$ and confirmed below, that this is not the case; an individual SC can be reliably measured only by blocking the second pump. 


\section{2: Results and discussion}

\subsection{1: Shift and injection currents}

A SC can be induced by a single optical pump field $\hbar \omega>E_{g}$ or $2 \hbar \omega>E_{g}$. The charge distribution in real space associated with a CB state is usually displaced from the charge distribution associated with the VB state at the same position in the Brillouin zone; one charge distribution might be centered on the $\mathrm{Bi}$ atoms, and the other on the Se atoms, for example. During absorption, it is then possible for the charge distribution to evolve from that of the VB state to that of the CB state in such a way that there is a net current associated with the change of state. This occurs most typically if the pump field vector points from one atom to the neighboring atom (red vector in Figure 5.1(c)). In the other polarization shown by the blue vector, there is no preference for the direction of the charge motion and no net SC results.

The lowest order contribution to the SC is governed by a third rank tensor, and associated with a divergent part of $\chi^{(2)}{ }^{45,58}$ It can be computed in terms of the incident field $E_{\omega}$ through a third-rank tensor $v_{a b c}$ given by

$$
J_{a}^{\text {shift }}=v_{a b c} E_{-\omega}^{b} E_{\omega}^{c}+\text { c.c. }
$$

The tensor $v_{a b c}$ satisfies the same symmetries as the crystal lattice. It vanishes for centrosymmetric materials such as $\mathrm{Bi}_{2} \mathrm{Se}_{3}$, but inversion symmetry is broken at the surface and $v_{a b c}$ can have non-vanishing components. For $\mathrm{SS}$ in $\mathrm{Bi}_{2} \mathrm{Se}_{3}$, the only non-zero tensor component is $v_{x x x}$. The parallel $(\alpha=\beta)$ component of the current $J_{\|}$, and the perpendicular $(\alpha=\beta+\pi / 2)$ component $J_{\perp}$ can be written as

$$
\begin{gathered}
J_{\|}^{\text {shift }}=v_{x x x} \cos (3 \beta)\left|E_{\omega}\right|^{2}, \\
J_{\perp}^{\text {shift }}=-v_{x x x} \sin (3 \beta)\left|E_{\omega}\right|^{2} .
\end{gathered}
$$

Similar terms exist for excitation with $\left|E_{2 \omega}\right|^{2}$.

To induce an IC one must use two phase-related pumps which overlap in time and space, which can have energies $\hbar \omega$ and $2 \hbar \omega$, and can be described by the phase parameter $\Delta \phi$. ICs occur as a result of quantum interference between 1PA and 2PA pathways creating an interference pattern in momentum 
space, and hence an asymmetric distribution of excited electrons. The lowest order contribution to the IC is associated with a divergent part of $\chi^{(3)}$, and governed by a fourth rank tensor $\eta_{a b c d}$ given by

$$
\frac{d}{d t} J_{a}^{i n j}=\eta_{a b c d} E_{-\omega}^{b} E_{-\omega}^{c} E_{2 \omega}^{d} e^{i \Delta \phi}+c . c .
$$

For both the surface and bulk of $\mathrm{Bi}_{2} \mathrm{Se}_{3}$, there are three independent components of $\eta$, namely $\eta_{x x x x}, \eta_{x y y x}$ and $\eta_{x y x y}$. The injection rate of the current along the direction of the incident field $(\alpha=\beta)$ is

$$
\frac{d}{d t} J_{\|}^{i n j}=2\left[\operatorname{Re}\left(\eta_{x x x x}\right) \cos (\Delta \phi)+\operatorname{Im}\left(\eta_{x x x x}\right) \sin (\Delta \phi)\left|E_{\omega}\right|^{2}\left|E_{2 \omega}\right|^{2}\right.
$$

while the component of the IC perpendicular to the incident field $\left(\alpha=\beta+\frac{\pi}{2}\right)$ vanishes $\frac{d}{d t} J_{\perp}^{i n j}=0$.

\subsection{2: Observation of competing current sources}

Figure 5.2 shows the THz emission versus $t_{d e t}$ and $\Delta \phi$ in parallel detection configuration $(\alpha=\beta)$. In (a), $\beta=\pi / 6$ results in suppression of the SC. With the sample rotation at $\beta=\pi / 6+n \pi / 3$ (where $n$ is an integer) no signal at $\alpha=\beta$ occurs for a one-color excitation. The checkerboard pattern of behavior in this case is similar to other IC experiments on GaAs, silicon, and graphene. 50,52,145,146 The THz transients shown in the inset are averages of the horizontal lines at $\Delta \phi=3 \pi / 2+2 \pi n$ (white) and at $\Delta \phi=\pi / 2+$ $2 \pi n$ (gray). They follow from the dynamics of the IC alone.

In (b) $\beta=0$ and shift and ICs are simultaneously observed. For $\beta=0+n \pi / 3$ radians, maximum SCs occur along $\alpha=\beta$ because a neighboring atom is aligned with the pump polarization. This behavior follows from Equation 5.2. The THz transient shown in the inset is an average of all of the horizontal lines from $\Delta \phi=-\pi / 2$ to $\Delta \phi=7 \pi / 2$. Averaging over a multiple of $2 \pi$ removes the oscillating IC, and therefore the white line follows from the dynamics of the SC alone. It contains contributions from both the pump at $\omega$ and that at $2 \omega$. 

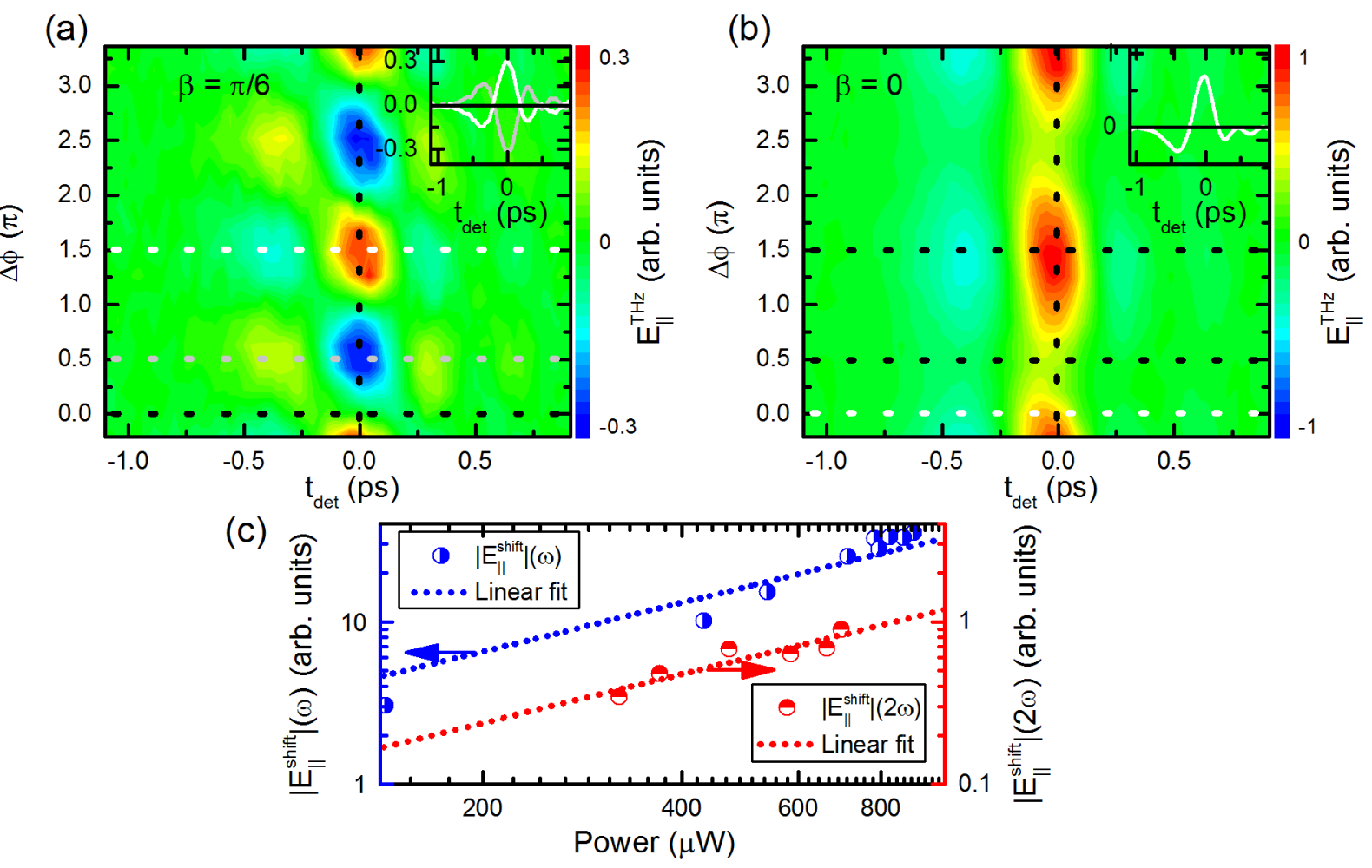

Figure 5.2: THz electric field emitted by $\mathrm{Bi}_{2} \mathrm{Se}_{3}$ as a function of $t_{d e t}$ and $\Delta \phi$. The dotted vertical line indicates $t_{\text {det }}=0$, the maximum for which the later polar angle measurements are taken. Dotted horizontal lines indicate transients for $\Delta \phi=0, \pi / 2$, and $3 \pi / 2$. (a) Here $\beta=\pi / 6$, resulting in a vanishing SC so only the characteristic checkerboard pattern of the IC is visible. The gray and white lines in the inset indicate the evolution of the THz electric fields that follow from the ICs at $\Delta \phi=\pi / 2$ and $3 \pi / 2$. (b) Here $\beta=0$, resulting in a maximum positive SC offsetting the oscillating IC. The white line shown in the inset indicates the evolution of the THz electric field following from the SC. (c) Power dependence of the electric field produced by a single pulse $\omega$ (left) or $2 \omega$ (right).

In the far field, the idealized shape of the THz electric field follows the form of $d J / d t .^{147} \operatorname{In}$ a model system like GaAs, this leads to the injection and SC transients having different shapes, but the samples used here have additional scattering caused by imperfect growth and imperfect lattice matching. The finite bandwidth available from the detection scheme may also lead to distortions of the shape of the transient. These complications lead to the transients having similar shapes in these experiments, and the shape cannot be directly related to that predicted by an idealized theory.

The power dependence of the SCs is shown in Figure 5.2(c). The THz electric field (and hence the current) for both $\omega$ and $2 \omega$ excitation is linear in the pump power, as expected from Equation 5.1. The $2 \omega$ 
$\mathrm{SC}$ is approximately an order of magnitude weaker, due mainly to the difference in absorption cross section at the two energies.

In addition to shift and ICs, other effects such as photon drag, where momentum is transferred between photons and electrons via scattering, can play a role in the generation of photocurrents. Previous studies have taken care to demonstrate that such effects are negligible in materials of the type we consider. ${ }^{20,68}$

It is observed that even when $\tau$ is much too great for current injection to occur, the signal observed at $t_{d e t}=0$ is modified by the presence of the $2 \omega$ pulse. Figure 5.3 shows this phenomenon. Figure $5.3(\mathrm{a})$ shows the phase-dependent cross-correlation of the IC offset by the SC as green data, with a fit of the form $A \exp \left[-\frac{\tau^{2}}{0.36\left(\tau_{x}\right)^{2}}\right] \sin \left[\omega\left(\tau-\tau_{0}\right)\right]+1$ shown in red, where the FWHM of the cross-correlation is $\tau_{x}=0.28 \mathrm{ps}$, $\tau_{0}$ is an arbitrary phase shift, and $A$ represents the relative strength of the IC. Also shown are three positions of the $2 \omega$ pulse outside the current injection envelope, used below. When the $2 \omega$ pump is blocked, the $E_{\|}^{\text {shift }}(\omega)$ contribution can be seen as the dotted red line in Figure 5.3(b). However, this contribution is modified by the $2 \omega$ pump, even when it impinges the sample a full 3.2 ps ( $\sim 0$ pulse widths) before the $\omega$ pump. The $\mathrm{THz}$ signal observed is modified by approximately $20 \%$ in this case, and the modification increases steadily to $50 \%$ as $\tau$ becomes closer to 0 . This is probably due to free carrier absorption of the $\mathrm{THz},{ }^{148}$ and it indicates that a trivial solution of simply adding the two separate SCs together is not necessarily appropriate. In Figure 5.3(c), this pump-probe contribution is mapped out over the entire available range of $\tau$, and is fit with a pulse model of the form $A\left[1-\exp \left(-\frac{\tau-\tau_{0}}{\tau_{1}}\right)\right]^{P} \exp \left[-\frac{\tau-\tau_{0}}{\tau_{2}}\right]$, with rise time $\tau_{1}=0.06 \mathrm{ps}$ and decay time $\tau_{2}=4.09 \mathrm{ps} ; A$ and $P$ are arbitrary fitting parameters to match the signal strength and $\tau_{0}$ is an arbitrary temporal shift. Any signal occurring at $\tau>0$ was ignored for this fit. 
(a) $\Delta \phi(\pi)$

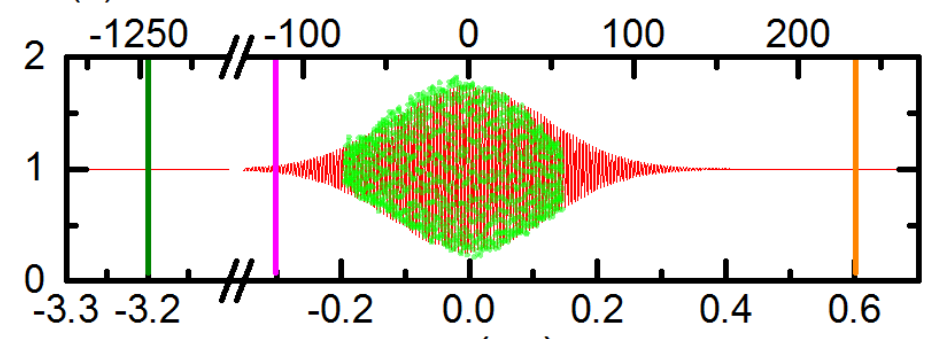

(b) $\tau(\mathrm{ps})$

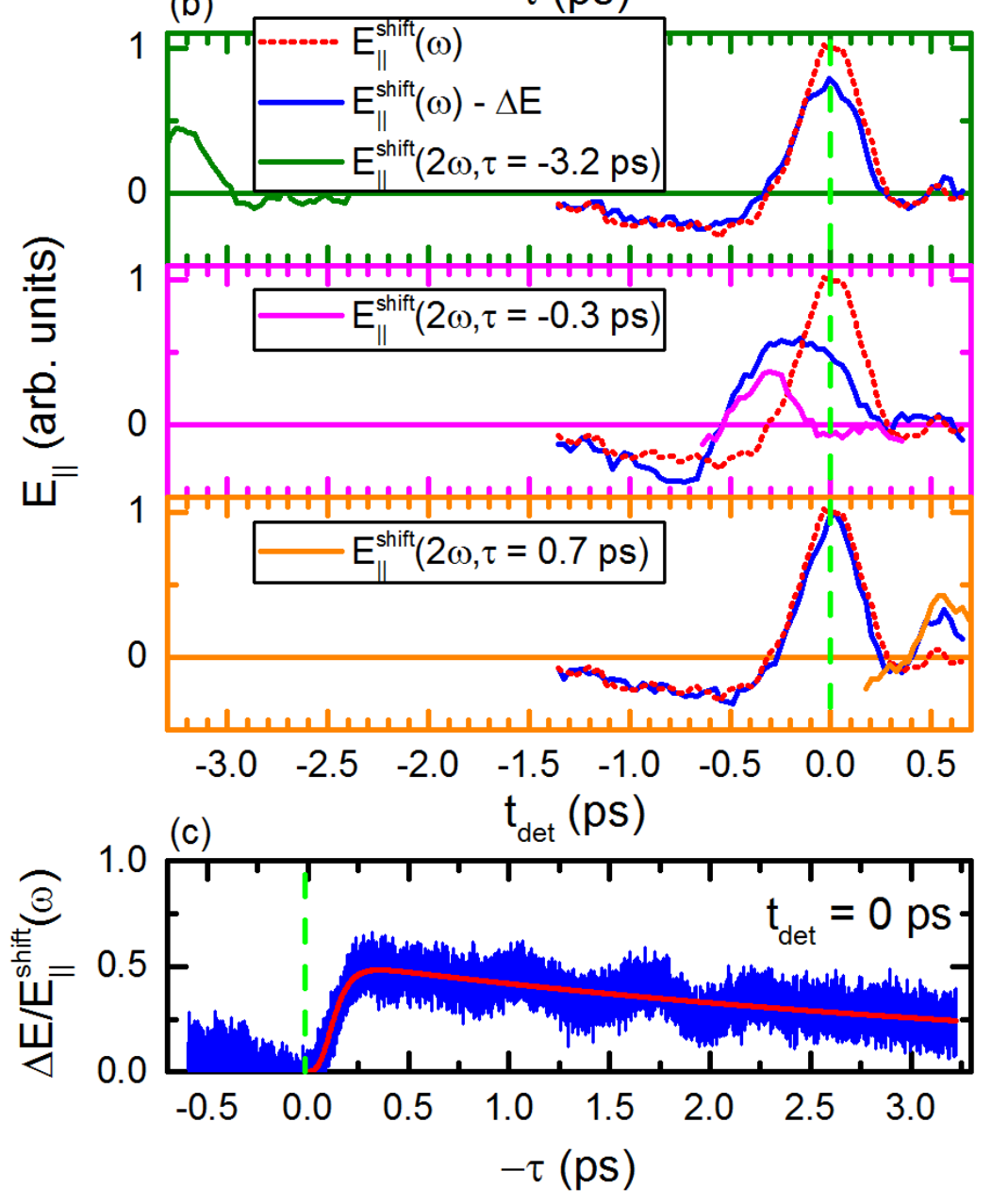

Figure 5.3: Measured electric field from SCs as a function of $\tau$. (a) The range of $\tau$ values with best overlap and maximum IC occurring at $\tau=0$, with vertical lines indicating the $2 \omega$ pulse occurring before, at the onset, and after the $\omega$ pulse, conditions shown in (b). The dotted red lines in (b) show $E_{\|}^{\text {shift }}(\omega)$, the $\mathrm{THz}$ electric field resulting from the SC, measured when only $\omega$ is present. The blue lines in (b) show how the field is modified by the presence of $2 \omega$ at three delay times. This behavior at fixed time $t_{d e t}$ over the entire range of $\tau$ values is shown in (c). 


\subsection{3: Separation of shift and injection currents}

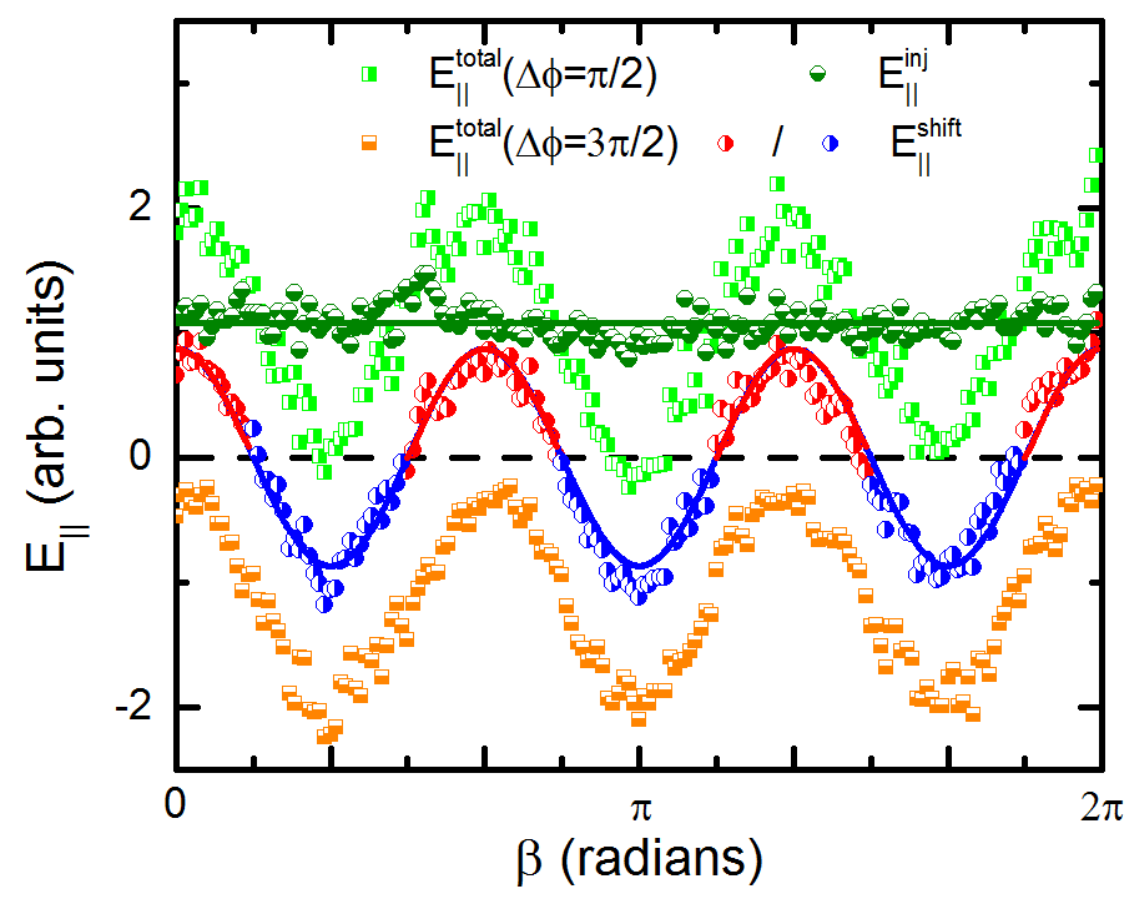

Figure 5.4: Demonstration of current separation analysis based on Equation 5.8. Light green is the two-color photocurrent at $\Delta \phi=\pi / 2$ and orange is the same at $\Delta \phi=3 \pi / 2$. Subtraction gives the IC in olive, and addition gives the SC in red/blue. Solid lines for $E^{\text {shift }}$ and $E^{\text {inj }}$ are theoretical models fit to experimental amplitude.

The total current measured by the THz probe is a sum of the shift and ICs, and while the IC depends on the phase parameter $\Delta \phi$, SCs are independent of it. Since $J_{\|}^{i n j}\left(\Delta \phi=\frac{\pi}{2}\right)=-J_{\|}^{i n j}\left(\Delta \phi=\frac{3 \pi}{2}\right)$, if the total current is measured for both values of the phase parameter

$$
\begin{gathered}
J_{\|}^{\text {total }}\left(\Delta \phi=\frac{\pi}{2}\right)=J_{\|}^{\text {shift }}+J_{\|}^{\text {inj }}\left(\Delta \phi=\frac{\pi}{2}\right), \\
J_{\|}^{\text {total }}\left(\Delta \phi=\frac{3 \pi}{2}\right)=J_{\|}^{\text {shift }}+J_{\|}^{\text {inj }}\left(\Delta \phi=\frac{3 \pi}{2}\right),
\end{gathered}
$$

it is possible to extract both the injection and SCs as

$$
\begin{gathered}
J_{\|}^{\text {inj }}\left(\frac{\pi}{2}\right)=\frac{1}{2}\left[J_{\|}^{\text {total }}\left(\frac{\pi}{2}\right)-J_{\|}^{\text {total }}\left(\frac{3 \pi}{2}\right)\right], \\
J_{\|}^{\text {shift }}=\frac{1}{2}\left[J_{\|}^{\text {total }}\left(\frac{\pi}{2}\right)+J_{\|}^{\text {total }}\left(\frac{3 \pi}{2}\right)\right] .
\end{gathered}
$$


In Figure 5.4 this treatment is demonstrated, showing that the IC is indeed $\beta$-independent, while the sum of the SCs follows the same trend as the individual SCs.

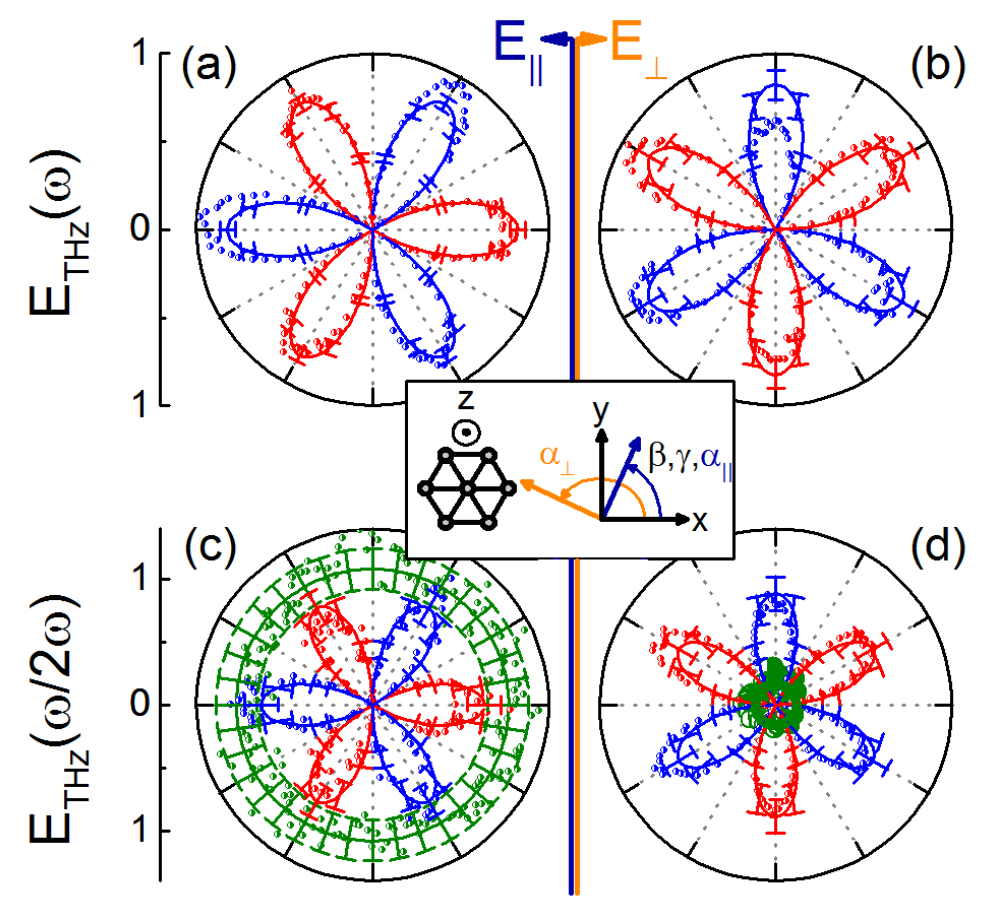

Figure 5.5: THz traces as a function of $\beta$, all obtained at time $t_{\text {det }}=0$. Inset shows the relevant angles. Red and blue lines in (a) - (d) indicate positive and negative values, respectively. In (a) and (c), the parallel $(\alpha=\beta)$ radiation was detected, and in (b) and (d), the perpendicular $(\alpha=\beta+\pi / 2)$ was detected. In (a) and (b), we see the current produced by the $\omega$ pump alone. In (c) and (d), both pumps were incident simultaneously and the shift and IC contributions were calculated using Equation 5.8. Error bars (15\% maximum for two-color measurements) were obtained by repeating scans multiple times and recording the difference in amplitudes for the sinusoidal fits. All data have been normalized to the magnitude of $E_{\|}^{\text {shift }}(\omega)$. Solid lines are theoretical models with magnitudes fitted to the data where SCs are assumed to be equal in parallel and perpendicular detection, and interference current is assumed to be zero in perpendicular detection.

The same $\beta$-dependence stemming from the lattice symmetry is reproduced by all SCs measured (see Figure 5.5). The peak amplitudes of the SCs with polarization parallel to the polarization of the $\omega$ and $2 \omega$ pulses can each be fit well by a cosine of the form $A \cos \left[\frac{2 \pi \beta}{T}\right]$ where $T=2 \pi / 3$, shown as solid lines in the figure. The peak amplitudes of the SCs with polarization perpendicular to the polarization of the $\omega$ and $2 \omega$ pulses are fit using $A \cos \left[\frac{2 \pi\left(\beta-\frac{\pi}{6}\right)}{T}\right]$, following the relationship in Equation 5.2. The difference in magnitude between the parallel and perpendicular contributions is negligible within experimental uncertainty. 
In Figure 5.5(c) and (d) the SC and IC contributions are calculated using $\omega+2 \omega$ excitations (Equation 5.8). Azimuthal angle measurements were recorded for conditions of minimum and maximum IC $(\Delta \phi=\pi / 2$ and $3 \pi / 2$, respectively). To minimize the effects of phase drift (which was observed to be as low as one cycle per hour), measurements were recorded for $2 \pi / 3$ radians at a time, and the phase was reoptimized in between before continuing. After subtraction to obtain the IC contribution, the results were found to be approximately independent of angle, with a residual $4.2 \%$ anisotropy (fit amplitude/offset).

Going from the detection of $\mathrm{THz}$ radiation with polarization parallel to that of the incident pulses to that with polarization perpendicular to that of the incident pulses, we see that the SC pattern is rotated by

an angle of $\pi / 6$, and the magnitude is increased by only $\left(\frac{E_{\|}-E_{\perp}}{E_{\|}+E_{\perp}}\right)=1.8 \%$, well within experimental uncertainty. We also see a reduction in the IC of approximately $74 \%$. As described by Equation 5.5, ICs are typically along the direction of the pump polarization. The reduction trends toward the expected value of $100 \%$. Currents perpendicular to the pump polarization may arise due to the strong spin-orbit coupling in the material, as has been observed in other semiconductors. ${ }^{149}$

It is known that z-direction components will arise from non-normal excitation, which yields further interesting possibilities for the generation of photocurrents..$^{41,42}$ Their generation may also result due to the built-in electric field caused by band-bending near the surfaces. ${ }^{150}$ The experiments performed here were unable to detect currents traveling in the z-direction, but their presence cannot be ruled out.

\section{3: Summary}

Photocurrents are often used as tools for exploring interesting optoelectronic properties of semiconductors. In many systems, selection rules and sample geometry preclude some photocurrent mechanisms while allowing others. Although in certain cases both one-color and two-color photocurrents have been observed simultaneously, to date they have not been isolated and studied in-depth in a rich system like the distinctive bulk and SS energy bands in three-dimensional TIs.

We presented the results of an all-optical experiment analyzing photocurrents in high-quality thin films of $\mathrm{Bi}_{2} \mathrm{Se}_{3}$. Two laser pumps generate photocurrents in the samples, such that each laser field alone 
can induce an SC, and quantum interference between 1PA and 2PA processes gives rise to an IC when both pumps are incident. The induced photocurrents were detected through $\mathrm{THz}$ radiation.

The crucial problem of separating the different contributions to the photocurrent was resolved by analyzing the relative-phase and polarization dependence of the total photocurrent detected via $\mathrm{THz}$ emission. ${ }^{151}$ The trivial solution of adding and subtracting currents was shown to have limitations coming from interplay between the two pulses even outside the cross-correlation envelope. We solve this using a complete characterization of the two-color photocurrents, giving a powerful tool for further analysis of not only $\mathrm{Bi}_{2} \mathrm{Se}_{3}$, but other systems as well that allow multiple photocurrent contributions.

The dependence on the direction of the polarization of the incident fields was shown to match theoretical predictions based on the symmetry of the crystal lattice. This confirms the characteristics of the individual currents, and suggests that SCs, despite complicating the analysis of ICs, can be used for extracting further information about the prominent SS. For example, because the sample symmetry allows SCs only from the surfaces and ICs from the bulk, trends in the dependence of the SC on pump energy and sample thickness can be explored in further depth. This strategy has the potential to provide great insight into the optoelectronic properties of $\mathrm{Bi}_{2} \mathrm{Se}_{3}$. It provides a promising bridge linking angle-resolved photoemission spectroscopy and transport measurements. 


\section{Chapter 6: Optical Properties of $\mathrm{Bi}_{2} \mathrm{Se}_{3}$}

A complete understanding of the photocurrents injected into $\mathrm{Bi}_{2} \mathrm{Se}_{3}$ must necessarily include some of the basic properties of the material, namely the linear and nonlinear absorption properties at the relevant photon energies. To this end, this chapter is focused on the due diligence. Since the pump wavelengths used for the bulk of this work have been $\lambda_{\omega}=1540 \mathrm{~nm}$ and $\lambda_{2 \omega}=770 \mathrm{~nm}$, these are the wavelengths focused on in this chapter as well. However, a complete understanding of the topological SS must necessarily involve a broad set of photon energies which excite electrons below, to, and above the Dirac cone. Therefore, we begin preliminary photon dependence of the absorption parameters of the materials involved, but a thorough analysis of the ICs as they relate to absorption is beyond this thesis and will be described in future works.

IC are created through a combination of 1PA and 2PA of the pumps, so modeling of the currents based on the absorption coefficients should be possible. Since current injection works via the interference of 1PA and 2PA pathways of the second harmonic and fundamental pulses, respectively, the important parameters are the 1PA coefficient $\alpha_{2 \omega}$ of the second harmonic and the 2PA coefficient $\beta_{\omega}$ of the fundamental. In this chapter we attempt to make sense of the IC efficiency through the number of excitations produced by the individual absorption processes.

\section{1: Linear absorption}

Reflection and transmission percentage were measured in each sample at $770 \mathrm{~nm}$ and $1540 \mathrm{~nm}$. For $770 \mathrm{~nm}$, a Coherent LM2-vis power sensor was used, and for $1540 \mathrm{~nm}$, calibrated Ge photodiodes were used in conjunction with the lock-in amplifier. $P_{0}$ is the measured power of the full beam with no sample in place. Transmitted power $P_{T}$ was measured at normal incidence, and reflected power $P_{R}$ was measured at a sample incidence angle of about 5 degrees from the optical axis. In both cases the sample orientation was such that the incident light would hit the thin-film first and the substrate second. Transmission percentage is $T=P_{T} / P_{0}$, and reflection percentage is $R=P_{R} / P_{0}$. With the data, 1PA was calculated using Beer's Law 


$$
\begin{gathered}
P=P_{0}(1-R) e^{-\alpha L} e^{-\alpha_{s u b} L_{s u b}} \\
\alpha=-\frac{1}{L} \ln \left(\frac{T}{(1-R) e^{-\alpha_{s u b} L_{s u b}}}\right)
\end{gathered}
$$

where $L$ is the thickness of the $\mathrm{Bi}_{2} \mathrm{Se}_{3}$ film, $T$ is the measured transmission percentage, $R$ is the measured reflection percentage, $\alpha_{\text {sub }}=0.0019 \mathrm{~cm}^{-1}$ is the 1PA coefficient of the sapphire substrate ${ }^{152}$, and $L_{s u b}=0.05 \mathrm{~cm}$ is the thickness of the substrate.

The results are compiled in Figure 6.2. The results for $\alpha_{2 \omega}$ match closely to those obtained by Glinka et al. ${ }^{18}$ similarly approaching the bulk value of $2 \times 10^{5} \mathrm{~cm}^{-1} .{ }^{153}$ For thinner films, the 1PA coefficient increases, and correspondingly the penetration depth $1 / \alpha$ decreases to values that are similar to those of thin metal films $(<10 \mathrm{~nm})$. This behavior may be due to the relative increase in the contribution of the SS compared to the bulk states. If SS are only present in the first and last QLs, then the ratio of surface to bulk in the 6-QL film is $1 / 3$, whereas in the $40-Q L$ film the ratio is $1 / 20$. Clearly the thickest films will experience a relatively much weaker effect from the SS than from typical bulk semiconductor absorption.

In contrast, $\alpha_{\omega}$ has the opposite behavior, with the thickest films experiencing the higher 1PA coefficient. This is probably due to the additional energy states that are added with each extra layer of material, resulting in a larger density of available CB states.

\section{2: Nonlinear absorption}

\subsection{1: Two-photon absorption}

Typically, the 2PA coefficient $\beta$ for a given material is experimentally determined using an openaperture z-scan. ${ }^{154}$ The literature reveals that open-aperture z-scans have been performed on several types of $\mathrm{Bi}_{2} \mathrm{Se}_{3}$ samples, and the results show a typical signature of $\mathrm{SA}$ rather than TPA. ${ }^{19,23,24}$ Measurements in our lab confirmed these previous results, and further power dependence was performed in order to detect the characteristic rollover where the primary absorption mechanism switches from SA to 2PA. However, the onset of physical damage to the samples was observed at approximately $5 \mathrm{GW} / \mathrm{cm}^{2}$, before any $2 \mathrm{PA}$ 
signature revealed itself. Therefore, $\beta_{\omega}$ was unable to be determined in our samples using traditional methods.

To unequivocally determine the thickness dependence of $\beta_{\omega}$, a two-color pump-probe transient absorption technique was used, similar to measurements previously performed on graphene ${ }^{155-157}$ and other materials ${ }^{158}$ (see Figure 6.1(a)). The fundamental and second harmonic pulses were produced and sent through a Mach-Zehnder interferometer much like in the scheme for generating IC described in Chapter 4. However, in this case the second harmonic pulse was strongly attenuated and focused before being incident on the sample, being used as a probe. The pump and probe pulse were measured to have strengths of $I_{\omega}=17 \mathrm{GW} / \mathrm{cm}^{2}$ and $I_{2 \omega}=1 \mathrm{GW} / \mathrm{cm}^{2}$, respectively. The pump had a $1 / e^{2}$ radius of $r_{\omega}=635 \mu \mathrm{m}$, and the probe passed through the center of the pump pulse spot with $a 1 / e^{2}$ radius of $r_{2 \omega}=100 \mu \mathrm{m}$. This geometry was used to ensure that the probe would experience a relatively uniform pump intensity as it passed through the sample, which should provide the best possible contrast in transmission $\Delta T$. The probe transmission was measured as a function of time delay between pump and probe. With the probe incident earlier in time than the pump, the signal was calibrated to be zero using the lock-in balanced detection scheme. With the two beams overlapped in time, probe transmission reached a maximum. With this scheme, since the pump photon energy is only half that of the probe, the only meaningful interaction which can modify the transmission of the probe is CB states being filled via 2PA of the pump, resulting in the sample becoming more transmissive to the probe. Transients were recorded for all samples and integrated over time to compare the relative 2PA efficiencies. 


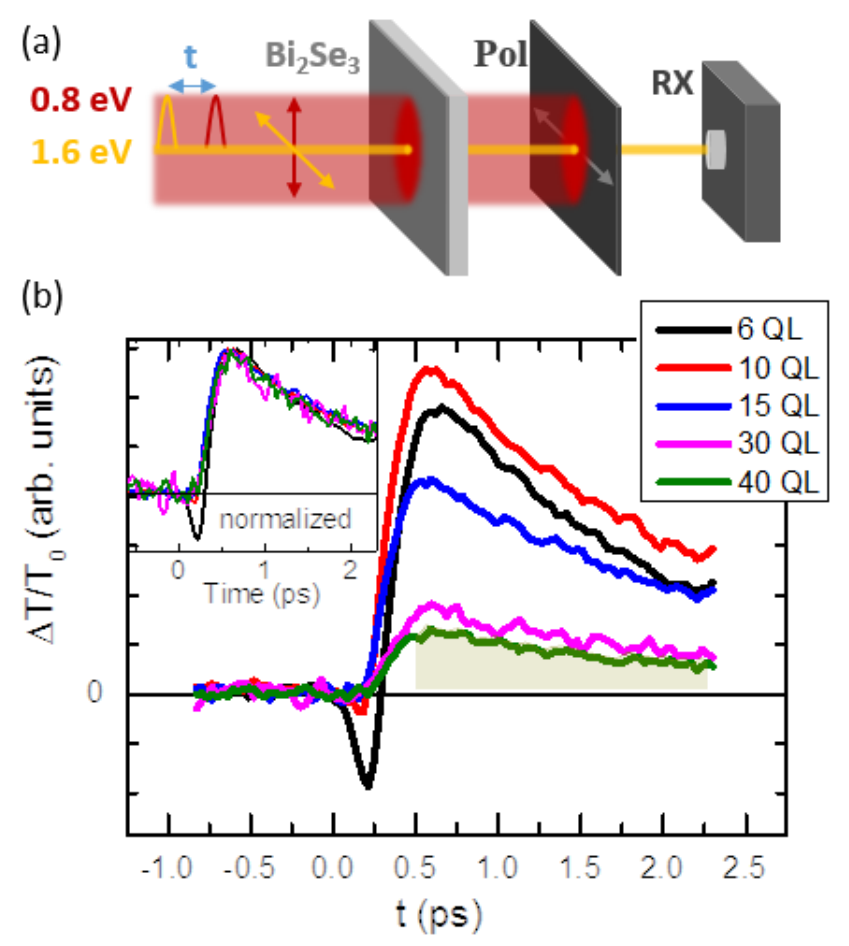

Figure 6.1: (a) Schematic for the experiment used to measure 2PA. The probe pulse (orange) is concentric with but smaller and more intense than the pump pulse (red). The pump and probe are cross-polarized so the pump can be blocked by the polarizer, and they are separated by a controllable time delay $t$. The transmitted power $P_{T}$ of the probe is measured by the detector RX. (b) Differential transmission of various sample thicknesses. In the inset, the data are normalized to the same maximum value so the shapes can be directly compared. The shaded region was integrated and used to compare $\beta$ across samples.

The detection scheme $(R X)$ is made up of balanced detection of two silicon photodiodes $A$ and $B$.

The transmitted intensity $(T)$ is measured with a detector A and lock-in amplifier. A portion of the probe is picked off with a beamsplitter before the sample and measured with a second silicon photodiode as a reference $\left(T_{0}\right)$. When there is zero time delay between the pump and probe pulses $(t=0)$, pump photons fill energy states at $1.6 \mathrm{eV}$ via $2 \mathrm{PA}$, which modifies the transmission of the probe pulses through the sample.

Chopping the probe with the pump blocked allowed for calibration of the detectors. With one arm of the probe passing through the sample to arrive at detector $A$, and the second arm passing through a variable ND filter to arrive at detector $B$, the signal could then be balanced by adjusting the ND filter until the measured signal A-B was zero. With the variable filter in the appropriate position, the chopper was then switched to the pump beam. By blocking detector B and using the auto-phase function of the lock-in 
amplifier, it was ensured that a positive measured A-B signal would correspond to an increase in intensity on detector $A$ with respect to detector $B$. Therefore, the sign of A-B is appropriately the same sign as $T-$ $T_{0}$.

The transient signals were observed to exhibit both positive and negative differential transmission $(\Delta T)$. As has been previously interpreted, ${ }^{157}$ a positive $\Delta T$ is caused by bleaching due to interband absorption of pump photons causing excited states to become filled. By contrast, the negative $\Delta T$ is a result of phonon-assisted intraband absorption and hence provides an unwanted contribution when determining the 2PA coefficient. When normalized to their respective maxima, 2PA transients all show identical bleaching and relaxation dynamics after the first $0.5 \mathrm{ps}$, so to remove the intraband absorption contribution and minimize noise the signals were integrated from $0.5-2.3 \mathrm{ps}$ and compared against each other. This result is proportional to the 2PA coefficient as a function of sample thickness, and is shown in Figure 6.2.

The 2PA coefficient at $1540 \mathrm{~nm}$ was calculated using the measured 1PA values and differential transmission data using

$$
\beta=\frac{\left(1-\frac{\Delta T}{T}\right) \alpha}{I_{P}(1-R)\left(1-e^{-\alpha L}\right)\left(1-r^{2} e^{-2 \alpha L}+\frac{\Delta T}{T}\right)}
$$

where $\beta$ is the TPA coefficient, $\Delta T / T$ is the measured differential transmission from the two-color pumpprobe experiment, and $I_{P}$ is the pump intensity. ${ }^{158}$ This treatment takes into account multiple reflections inside the sample and comes from the transmission of the probe measured at zero time delay between pump and probe pulses. $\beta$ is calculated to be on the order of about $4-12 \times 10^{4} \mathrm{~cm} / \mathrm{GW}$, values similar to those measured previously in graphene. ${ }^{159}$ 


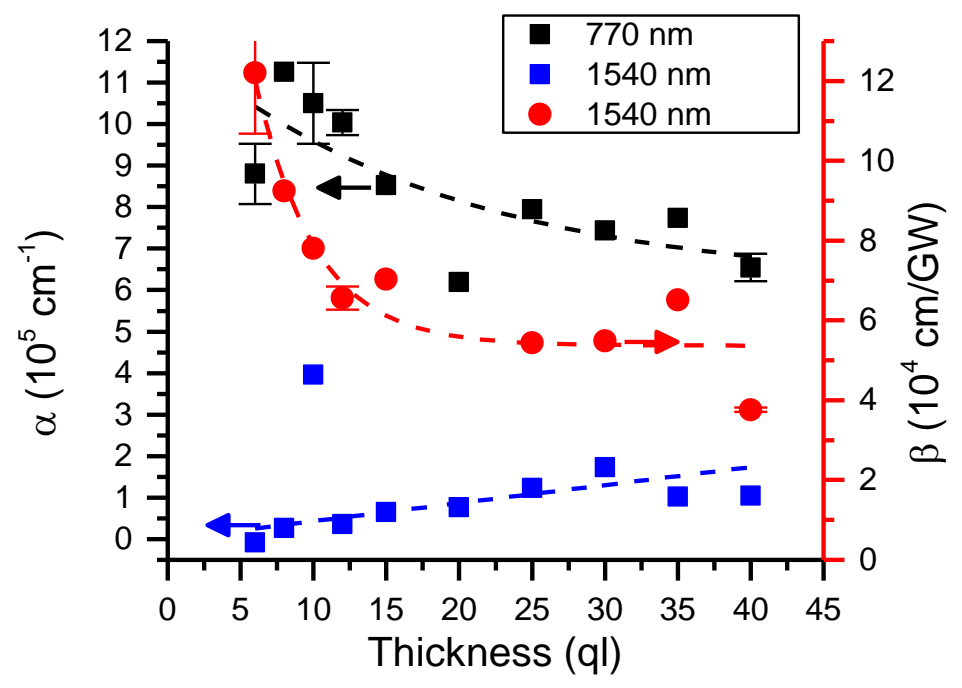

Figure 6.2: Measured values of 1PA (left axis) and 2PA (right axis) coefficients in various samples. Dashed lines are reduced chi-squared fits to the data.

$\alpha_{2 \omega}$ and $\beta_{\omega}$ were simultaneously considered as equal factors competing to determine the IC strength, as one photon at $2 \omega$ and two photons at $\omega$ must combine as a single fundamental unit to produce a current. When multiplied together, the dotted line in Figure 6.4 is produced. In thin samples $(<12 \mathrm{QL})$, the low $\alpha_{2 \omega}$ is the limiting factor, and in thick samples (>12 QL), the low $\beta_{2 \omega}$ is the limiting factor. This behavior is consistent with the IC, which appears to be less efficient in both thin and thick samples with a peak efficiency at around $12 \mathrm{QL}$.

\subsection{2: Beer's law model}

The measured absorption values were modeled using chi-square curve fitting (dashed lines in Figure 6.2), giving an exponential decay for $\alpha_{2 \omega}$ and $\beta_{\omega}$, and a straight line for $\alpha_{\omega}$. These values along with the measured reflection coefficients were then applied in the following layer-by-layer analysis on each sample. An initial intensity of $17 \mathrm{GW} / \mathrm{cm}^{2}$ and $1 \mathrm{GW} / \mathrm{cm}^{2}$ was used for both $I_{\omega}$ and $I_{2 \omega}$, respectively, and the intensity in layer $n$ was calculated with the Beer's law equation $I_{n}=I_{n-1} e^{-\left(\alpha+\beta I_{n-1}\right) L}$ where $L=1 \mathrm{~nm}$ is the thickness of a single layer. The difference in intensity between layers $n-1$ and $n$ was converted to the number of photons absorbed locally, and for the $\omega$ pump, the number of photons absorbed via 1PA and 2PA were calculated independently to be $N_{\omega, \alpha}$ and $N_{\omega, \beta}$. For the $2 \omega$ beam, the number of excitations 
produced at the desired energy level $2 \hbar \omega$ is simply $N_{2 \omega}$, and the number produced by the $\omega$ beam is $N_{\omega, \beta} / 2$. Results are shown in Figure 6.3.

The average powers measured in the injection experiment in Chapter 5 were $\bar{P}_{\omega}=35 \mathrm{~mW}$ and $\bar{P}_{2 \omega}=1 \mathrm{~mW}$. To calculate the power $P$ of an individual pulse, the average power is multiplied by the ratio of the time between pulses to the pulse duration in a square pulse approximation: $P=\bar{P}\left(\frac{1 \mathrm{~ms}}{100 \mathrm{fs}}\right)$. The pulse intensity is $I=P / A$, where $A=\pi r^{2}$ is the excited area, $r=0.75 \mathrm{~cm}$ being the $1 / e^{2}$-radius measured by the knife-edge technique. This gives pulse intensities $I_{\omega}=20 \mathrm{GW} / \mathrm{cm}^{2}$ and $I_{2 \omega}=0.56 \mathrm{GW} / \mathrm{cm}^{2}$.

The depth dependent intensity as a pulse passes through the sample is then modeled by Beer's law:

$$
I(z)=I_{0} e^{-\left(\alpha+\beta I_{0}\right) z}
$$

which can be explicitly modified for the $n$th QL:

$$
I_{n}=I_{n-1} e^{-\left(\alpha+\beta I_{n-1}\right) L}
$$

where $L$ is the thickness of a single QL (1 nm).

The amount of light absorbed between QLs $n-1$ and $n$ is $I_{n}$, which is equal to $N_{n}$ photons where

$$
N_{n}=I_{n} \tau_{\text {pulse }} A / E_{\text {photon }}
$$

with pulse duration $\tau_{\text {pulse }}$, area $A$, and photon energy $E_{\text {photon }}$. The ratio of the number of photons absorbed via $2 \mathrm{PA}$ to the number absorbed linearly is

$$
r_{a b s}=\frac{e^{-\beta I L}}{e^{-\alpha L}}
$$

Using these relations for the 12-QL sample we calculate the number of photons absorbed linearly by the first layer to be $N_{2}(\omega)=8.3 \times 10^{11}$ and $N_{2}(2 \omega)=2.0 \times 10^{11}$ respectively for the $\omega$ and $2 \omega$ pumps. In the last layer, $N_{2}(\omega)=4.4 \times 10^{11}$ and $N_{12}(2 \omega)=0.78 \times 10^{11}$. The amount of $\omega$ photons absorbed via $2 \mathrm{PA}$ is 
$6.0 \times 10^{12}$ and $1.7 \times 10^{12}$ in the first and last layers, respectively. These calculations assume that $2 \mathrm{PA}$ of the $2 \omega$ pump beam is negligible.

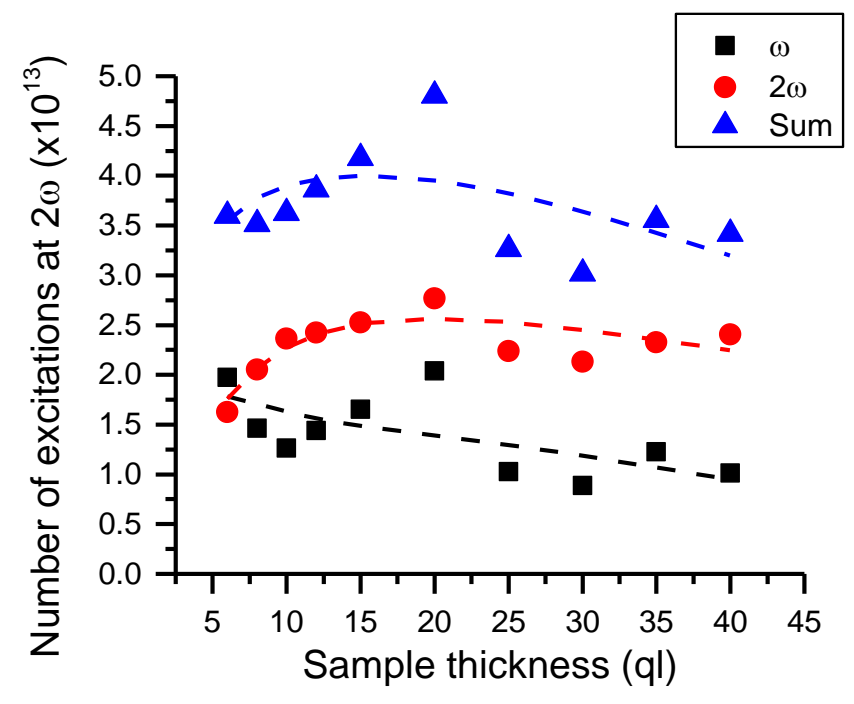

Figure 6.3: Number of excitations in the desired energy band $2 \hbar \omega$ produced by the $\omega$ beam (black), the $2 \omega$ beam (red), and the sum of the two contributions (blue).

The IC efficiency can be approximately modeled by the sum of excitations at $2 \hbar \omega$ produced by the $\omega$ beam and the $2 \omega$ beam. The result is shown in Figure 6.4 normalized and compared to the measured IC efficiency, demonstrating good agreement. The model could be further improved by taking into account how the local absorption in a given sample changes as a function of layer (with surface layers being more absorptive). 


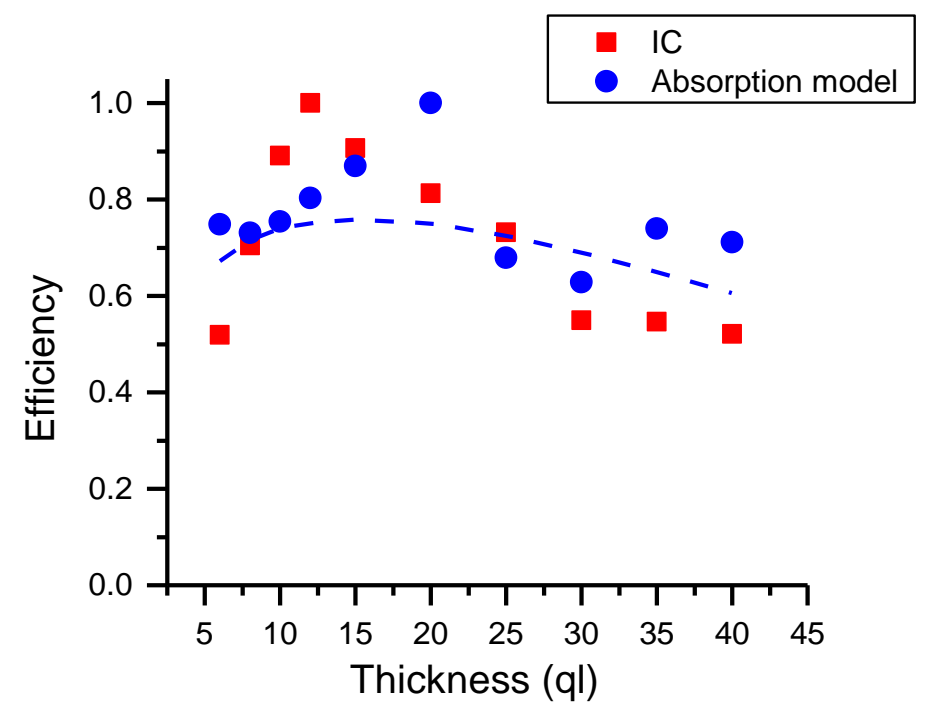

Figure 6.4: Red data points are the measured IC efficiency as a function of sample thickness. Blue data points are the values for total number of excitations at the desired energy band, and the dashed line is the same obtained by using the fits to the absorption data.

\subsection{3: Saturable absorption}

The z-scan is one of the most widely used methods of determining the nonlinear coefficients of a material because of its ease of use and high accuracy. ${ }^{154}$ The experiment is performed by bringing a beam to a focus, which has the effect of gradually increasing and then decreasing the intensity as a sample is scanned along the optical axis through the focus.

In the open aperture z-scan, all of the transmitted light is collected by a detector. Therefore, the experiment is only sensitive to the sample's absorption $\alpha$, which is given as a function of intensity $I$ in terms of both the SA and 2PA:

$$
\alpha(I)=\frac{\alpha_{0}}{1+I / I_{S}}+\beta I
$$

where $\alpha_{0}$ is the 1PA coefficient, $I_{S}$ is the saturable intensity, and $\beta$ is the 2PA coefficient.

The 2PA scales linearly with intensity, so when the sample is at the focus, absorption reaches a maximum. This causes a peak in the transmission, and the feature can be modeled by

$$
T(x, t)=\exp \left(\alpha_{0} L\right)\left[1+\sum_{m=1}^{\infty} \frac{\left(-\alpha_{0} L\right)^{m}}{m !} q_{m}(\rho)+\sum_{m=1}^{\infty} \frac{\left(-\beta L I_{S}\right)^{m}}{m !} q_{m}^{\prime}(\rho)\right]
$$


where $x=z / z_{0}$ is the relative distance of the sample from the focal plane and $L$ is the sample thickness. ${ }^{160}$ From this fitting function the saturable intensity and 2PA coefficient can be extracted.

Usually, $I_{S}$ is ignorable and $\beta$ is straightforwardly obtained from the z-scan. However, in some materials, 2PA is at odds with SA, in which an energy band is populated at a faster rate than it can deplete, causing more excited states to become filled on average, and therefore allowing less absorption at the target energy. In such a material, the open aperture z-scan results in a valley rather than a peak at the focus. As intensity is increased, 2PA overcomes SA more quickly, and the valley becomes a peak.

(a)

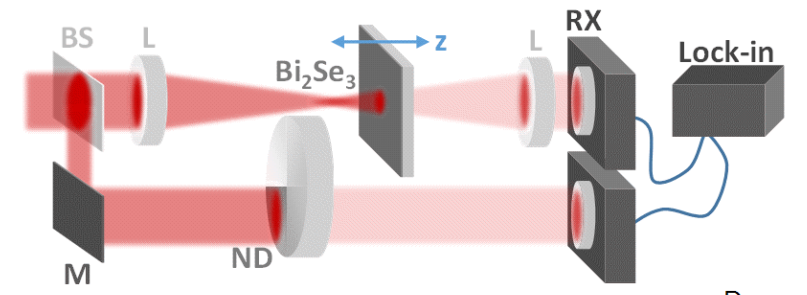

(b)

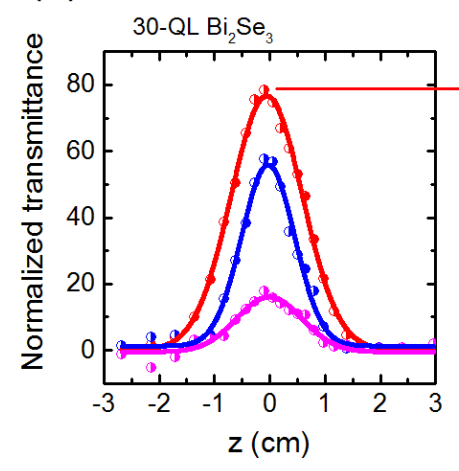

(c)
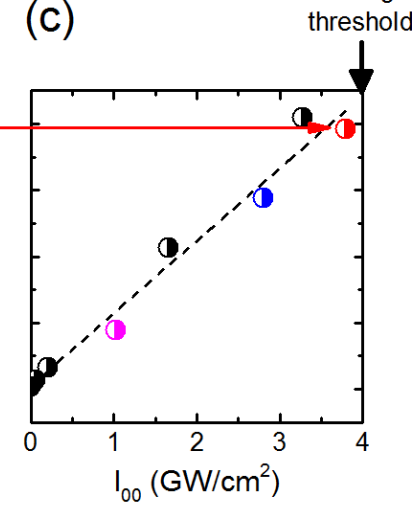

Figure 6.5: (a) Schematic for z-scan measurement of SA coefficient. $B S=$ beamsplitter; $L=$ lens; $M=$ mirror, $N D=$ neutral density filter; $R X$ = detection photodiode. The sample is moved along the $z$-axis through the focus of the laser. (b) Normalized transmittance through the sample as a function of $z$ position. When the beam is most intense, the sample becomes more transmissive. (c) Power dependence of the z-scan measurement, showing linear behavior up to the damage threshold of the sample.

$\mathrm{Bi}_{2} \mathrm{Se}_{3}$ is known to be an SA material and has even been proposed as a Q-switching material in fiber lasers to exploit this property. ${ }^{19,23,24}$ Essentially, as laser energy builds up in the cavity, it slowly reaches a point where the material becomes transmissive, at which point a single pulse of photons is allowed to 
leave the cavity. After it exits, the lack of energy within the cavity causes the Q-switching material to again become opaque to the light.

Figure 6.5(a) shows the experiment used to measure $\mathrm{SA}$ of $\mathrm{Bi}_{2} \mathrm{Se}_{3}$. A 1540-nm pulse was focused with a 20-cm lens, giving a Rayleigh length $z_{R}=0.41 \mathrm{~mm}$ and a beam waist of $w_{0}=142 \mu \mathrm{m}$. Pump power was attenuated to achieve maximum intensity at the beam waist of $0-4 \mathrm{GW} / \mathrm{cm}^{2}$, after which the intensity was high enough to permanently damage the sample.

\section{3: Photocurrent efficiency in $\mathrm{Bi}_{2} \mathrm{Se}_{3}$ and $\mathrm{GaAs}$}

To develop a sense of the efficiency of current injection in $\mathrm{Bi}_{2} \mathrm{Se}_{3}$, we use 1- $\mu \mathrm{m}$ thick (100)-grown GaAs as a reference. GaAs is a good starting point for comparison because it has already been used extensively in current injection experiments. The relevant coefficient for $1 \mathrm{PA}$ in GaAs is $\alpha_{2 \omega}^{\mathrm{GaAs}}=1.5 \times$ $10^{4} \mathrm{~cm}^{-1}$, and for $2 \mathrm{PA}$ is $\beta_{\omega}^{\mathrm{GaAs}}=5 \mathrm{~cm} / \mathrm{GW} .{ }^{145}$ Measurements of these coefficients in $\mathrm{Bi}_{2} \mathrm{Se}_{3}$ revealed

$\alpha_{2 \omega}^{\mathrm{Bi}_{2} \mathrm{Se}_{3}}=1 \times 10^{5} \mathrm{~cm}^{-1}$ and $\beta_{\omega}^{\mathrm{Bi}_{2} \mathrm{Se}_{3}}=5 \times 10^{4} \mathrm{~cm} / \mathrm{GW}$. The relative efficiency of each absorption process is then $\eta_{\alpha}=\alpha_{2 \omega}^{\mathrm{Bi}_{2} \mathrm{Se}_{3}} / \alpha_{2 \omega}^{\mathrm{GaAs}}=6.7$ and $\eta_{\beta}=\beta_{\omega}^{\mathrm{Bi}_{2} \mathrm{Se}_{3}} / \beta_{\omega}^{\mathrm{GaAs}}=10,000$. Since both processes need to be simultaneously present to produce ICs, the limiting factor in current injection efficiency is $\eta_{\alpha}=6.7$.

Also important in the determination of two-color current injection efficiency is group velocity mismatch (GVM). The refractive indices of GaAs at the relevant frequencies are $n_{\omega}=3.374$ and $n_{2 \omega}=$ $3.70 .{ }^{161} \mathrm{GVM}$ is given by $G V M=1 / v_{\omega}+1 / v_{2 \omega}=1.1 \mathrm{fs} / \mu \mathrm{m}$ (where the velocities $v_{\omega}$ and $v_{2 \omega}$ are given by $\left.v_{\omega, 2 \omega}=c / n_{\omega, 2 \omega}\right)$. Therefore, by the time the fundamental and second harmonic pulses travel through the sample, they will become out of phase by $1.1 \mathrm{fs}$, or $\sim 1 / 5$ of a cycle at the fundamental frequency. This mismatch has the effect of reducing the overall IC, but it is not enough to destroy it completely.

To compare the efficiencies of the various photocurrents for the pump photon energies used in Chapters 4 and 5, the maximum $\mathrm{THz}$ amplitude for each process was normalized to the pump intensity. The SCs for the fundamental and second harmonic $\left|E^{\text {shift }}\right|(\omega)$ and $\left|E^{\text {shift }}\right|(2 \omega)$ and the two-color IC $\left|E^{\text {inj }}\right|$ were obtained in Section 5.2.3. The amplitudes of these normalized to pump intensity are shown as the left three columns in Figure 6.6. The right three columns are the same experiments performed in GaAs: An IC 
was obtained with similar amplitude to the $\mathrm{Bi}_{2} \mathrm{Se}_{3}$, but if any SCs were present they were too weak to be detected by the experiment here.

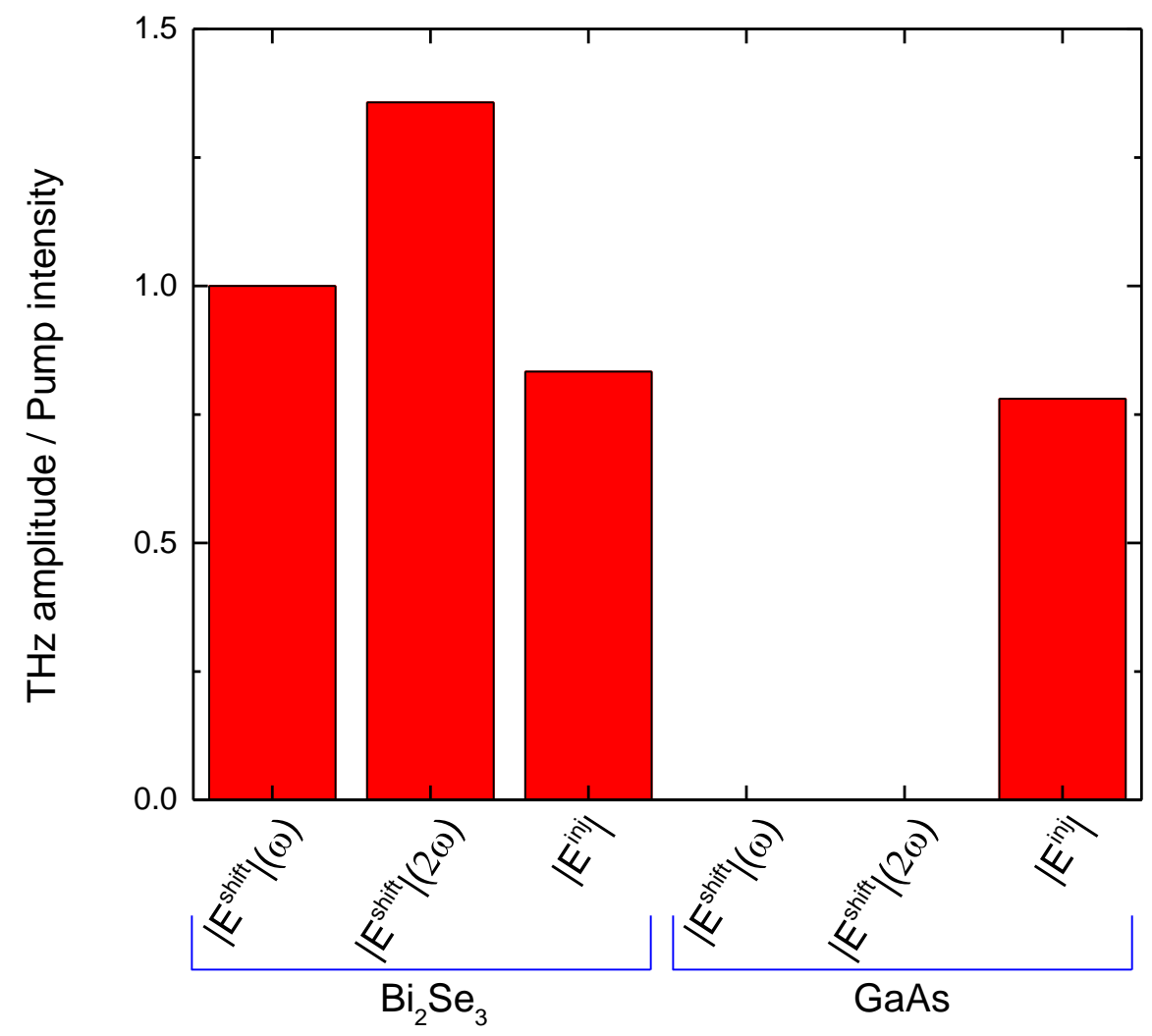

Figure 6.6: $\mathrm{THz}$ amplitude comparison between $\mathrm{Bi}_{2} \mathrm{Se}_{3}$ and $\mathrm{GaAs}$.

To explain this behavior, the relevant excitation densities for each process were calculated and shown in Figure 6.6. The excitation density in number of photons per volume is calculated with

$$
\begin{aligned}
N^{1 \mathrm{PA}} & =\frac{I_{0}(1-R)\left(1-e^{-\alpha L}\right) \tau}{L \hbar \omega} \\
N^{2 P A} & =\frac{I_{0}(1-R)\left(1-e^{-\beta I_{0} L}\right) \tau}{2 L \hbar \omega} .
\end{aligned}
$$

Here, $I_{0}$ is the pump intensity, $R$ is the reflectivity, $\alpha$ and $\beta$ are the 1PA and 2PA coefficients, respectively, $L$ is the thickness of the sample, $\tau$ is the duration of the pump pulse, and $\hbar \omega$ is the pump photon energy. The factor of 2 appears in the case of 2PA because two photons are required to produce one excitation. 


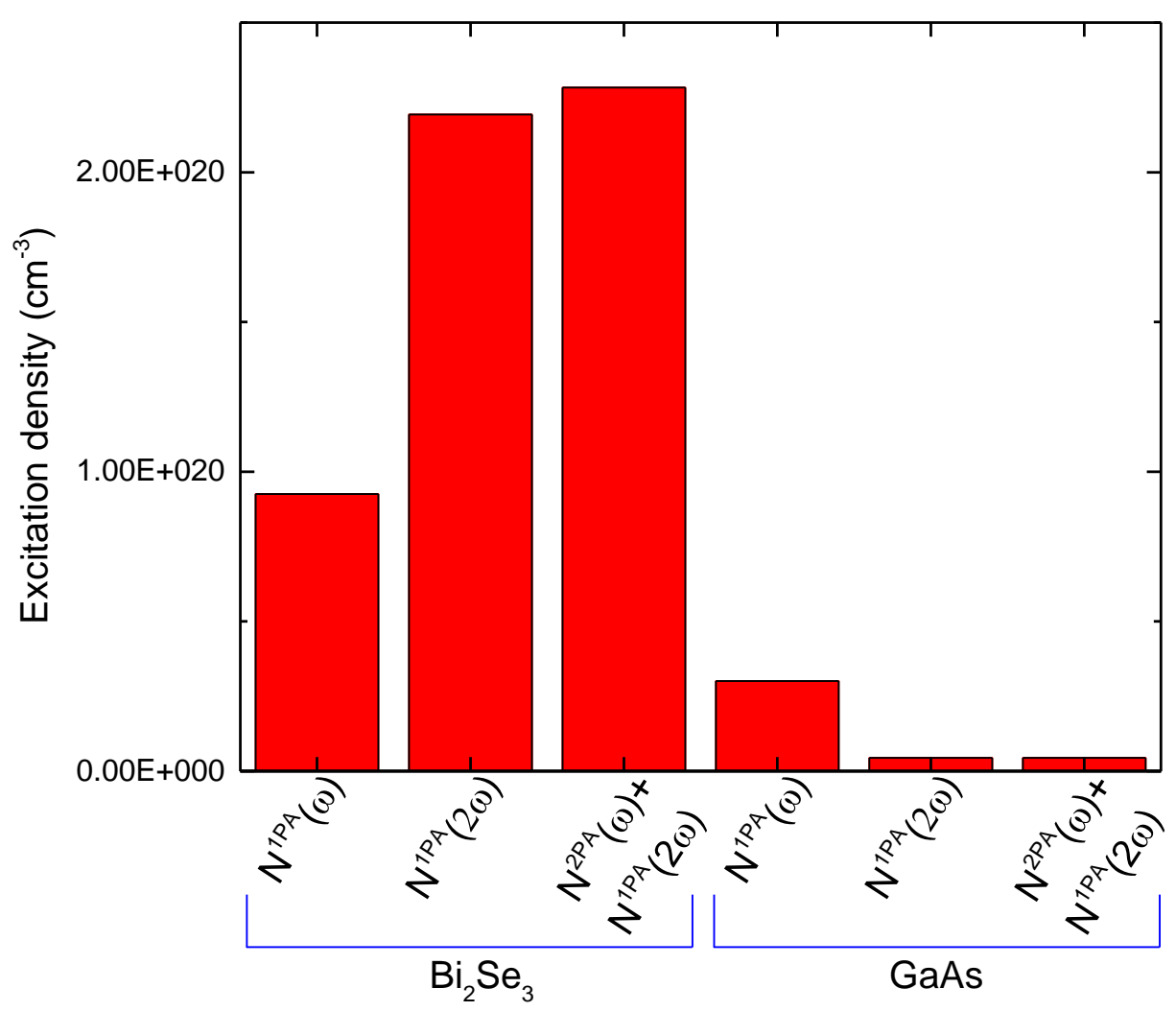

Figure 6.7: Excitation densities generated in $\mathrm{Bi}_{2} \mathrm{Se}_{3}$ and $\mathrm{GaAs}$ based on absorption coefficients.

From this analysis it was determined that the number of excitations per volume produced in $\mathrm{Bi}_{2} \mathrm{Se}_{3}$ that can be used for current injection is about 50 times higher than that produced in GaAs. These excitations are produced throughout the entire bulk of a sample, and the GaAs sample used was about 100 times thicker than the $\mathrm{Bi}_{2} \mathrm{Se}_{3}$. Therefore, these factors roughly cancel each other and the IC magnitude ends up being similar in both samples.

This comparison of excitation densities is only a starting point towards a full understanding. In reality, a single absorption coefficient cannot be used to describe absorption in both the surfaces and bulk of a TI because carriers in each behave very differently. However, this rough estimate provides evidence that 2PA of a fundamental pulse and 1PA of its second harmonic seem to be the dominant processes that work together to produce ICs, and with further characterization experiments will come more detailed analysis. 


\section{4: Summary}
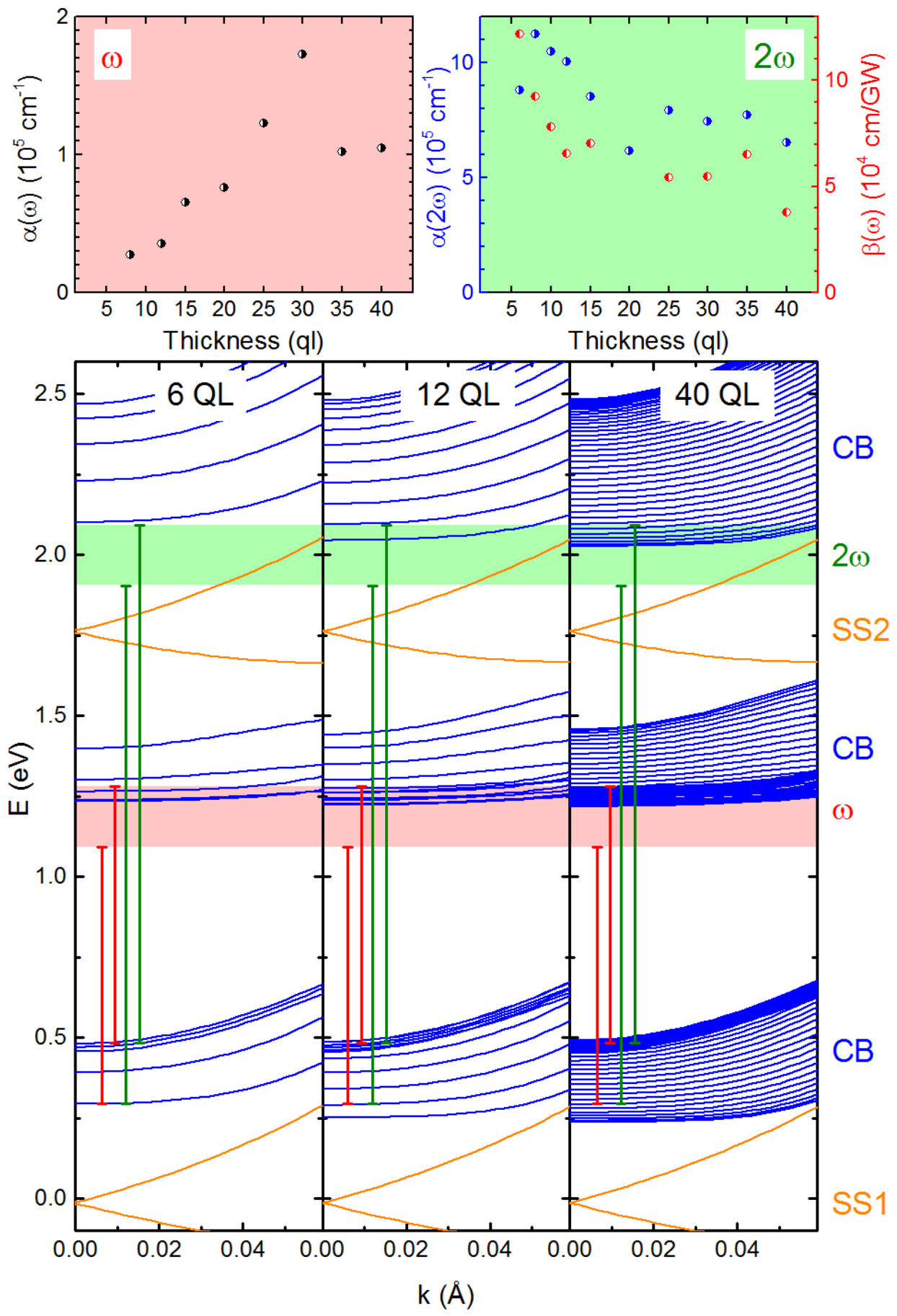

Figure 6.8: Compilation of absorption data and its relation to the band structure. The red vertical lines indicate roughly where an electron would start and end after stimulation by energy $\hbar \omega$. The green vertical lines indicate the same for $2 \hbar \omega$. 
The linear and nonlinear absorption of various thicknesses of $\mathrm{Bi}_{2} \mathrm{Se}_{3}$ films was measured and compared to previous results for IC efficiencies. Figure 6.8 shows how the results relate to the band structure as a function of thickness. As is usual in thin-film semiconductors, the addition of more layers of material results in additional energy states in the band structure. The vertical lines illustrate excitation with the pump energies used in Chapters 5 and 6 . A multitude of transitions are possible, from SS1 to SS2, SS1 to bulk, bulk to SS2, or bulk to bulk. Systematically lowering the photon energy should create transitions to a state where SS2 is being accessed more efficiently, until it can no longer be accessed at all due to the excitation energy being insufficient. Doing this pump dependence while repeating the experiments laid out in Chapters 5 and 6 should therefore reveal signatures that reflect differences in carrier dynamics between bulk states and TI SS. 


\section{Chapter 7: Conclusions}

As Moore's Law continues to approach fundamental limits, the need emerges for paradigm shifts in the approaches used in the technology of computing. Rather than continue along the same path of making similar components smaller and faster, we look towards peripheral aspects and unexplored parameters. Thus comes the need to manipulate electronic spins and utilize them as a novel aspect in computing. But with the necessity of new paradigms comes the great responsibility to the scientific community to nail down a solid foundation of understanding. What materials can we use to create useful devices? What are the best options for these materials? How do we efficiently characterize them? These are open questions that must be explored in the development of new technology.

We have explored in this work how $\mathrm{THz}$ radiation is exploited in the study of charge and spin carrier dynamics in novel materials. The groundwork has been laid for $\mathrm{THz}$ generation and detection, and the limitations- including the atmospheric absorption lines, and the difficulty of accurate transformation of $\mathrm{THz}$ waveforms in free space, have been outlined. THz conductivity of free carriers is probed and used to determine that nitrogen-doping of LTO can be used to improve solar collection efficiency without creating mid-gap trapping states that could harmfully reduce carrier lifetimes. ${ }^{79}$

THz-TDS was demonstrated as a novel method to study magnons in antiferromagnets- a material class with vast potential in a variety of spintronics applications. In this work, $\mathrm{MnF}_{2}$ was used as a wellcharacterized model system to demonstrate proof-of-principle. The Néel temperature $(67 \mathrm{~K})$ and magnon resonance frequency $(0.3 \mathrm{THz})$ are readily accessible using these techniques, and the temporal dynamics and temperature dynamics were explored in depth. By transmitting $\mathrm{THz}$ through the material we directly observed the one-magnon resonance and the two-magnon excited-state exchange resonance simultaneously. The red-shifting of the resonance frequency as temperature increased was modeled using a modified molecular field theory. The model's fit to our data led to the conclusion that neighboring spins in $\mathrm{MnF}_{2}$ are only weakly correlated in the intermediate temperature range $20 \mathrm{~K}<T_{N}<50 \mathrm{~K}$, with the correlation coefficient $j=1.1$ agreeing with mean-field theory. To our knowledge this is the first time the 
coupling coefficient in an AF material has been directly observed through experiment, and the THz-TDS technique used to obtain it can easily be extended to measure the same in other AF materials.

In addition, the transient nature of the THz-TDS experiments was exploited to determine the $\mathrm{THz}$ refractive index as a function of temperature. Above the Néel temperature, we showed that the refractive index grows with an increase of phonons from thermal energy. Below, magnetic energy is the primary contributor to the refractive index. These two effects are well-understood and have been observed in MnO. ${ }^{112}$ However, at very low temperatures, hyperfine interactions have been predicted but not yet observed until this work. The application of THz-TDS to $\mathrm{MnF}_{2}$ provides the first direct observation of the hyperfine contribution to the $\mathrm{THz}$ refractive index. With access to a cryostat that can reach low enough temperatures, this technique may be analogously applied to other AFs in a similar fashion.

The other class of material thoroughly explored in this work has been Tls. Primarily due to the strong spin-orbit coupling from a very heavy atom, Tls are able to freely transport electrons along their surfaces even when their bulk is insulating. This and the fact that conducting surface electrons are spinlocked orthogonally to the momentum makes them extremely interesting for many spintronics applications, like spin-injection or spin-filtering. Many groups are studying carrier dynamics in Tls using a variety of experimental methods.

We chose to focus our studies on $\mathrm{Bi}_{2} \mathrm{Se}_{3}$, the prototypical $\mathrm{TI}$, because it is well-characterized and has a relatively simple band structure compared to some alternatives. Thin-film samples of thicknesses from 6-40 $\mathrm{nm}$ were grown by collaborators as a method of varying the relative contributions of surface electronic states and bulk states to experimental findings. Our aim has been to probe the rich band structure using available light-matter interaction techniques with a tunable ultrafast laser pump. Using 1540- and $770-\mathrm{nm}$ light, we injected for the first time coherently controlled ICs in TIs, ${ }^{64}$ which can be manipulated in magnitude and direction via the relative phase of two collinear pump beams. Injecting a controllable current into a material using light allows us to avoid unnecessary side-effects of having a metal-semiconductor interface (such as band-bending), and probe the dynamics of the injected currents by varying several parameters such as intensity and photon energy. 
Aside from the successful observation of two-pump ICs, we also observed electron dynamics caused by individual pump beams which we attributed to SCs. SCs are forbidden in bulk states of $\mathrm{Bi}_{2} \mathrm{Se}_{3}$ due to symmetry, so the signals we observed are known to come from carriers in SS. By varying the direction of the pump polarization relative to the crystal lattice, we isolated the individual SC contributions to the overall photocurrent signal from the IC, and demonstrated how multiple interactions can be simultaneously observed and then later deconstructed into individual components.

As a further means of understanding the photocurrents we observed in $\mathrm{Bi}_{2} \mathrm{Se}_{3}$, we studied the absorption coefficients as a function of photon energy and thickness. SA was observed along with both linear and nonlinear absorption, and the relevant coefficients were recorded. The thickness dependence of the absorption coefficients appears to accurately describe the thickness dependence we observed in the ICs. The photon energy dependence relates directly to the band structures. This map of absorption coefficients goes one step further to a complete understanding of the carrier dynamics in $\mathrm{Bi}_{2} \mathrm{Se}_{3}$ and thus TIs in general. We then used these measurements to provide a starting point of comparison between the efficiency of photocurrent processes in $\mathrm{Bi}_{2} \mathrm{Se}_{3}$ and the more common semiconductor $\mathrm{GaAs}$.

With the addition of new experiments and uses for $\mathrm{THz}$ comes a need for $\mathrm{THz}$ devices such as waveguides, that we might control it in analogous ways to radiation on either side of the $\mathrm{THz}$ spectrum. To this end, a semiconductor optical fiber grown with a molten silicon core and a silica cladding was proposed as a commercial, mass-producible $\mathrm{THz}$ waveguide. Two-dimensional simulations using Maxwell's equations were used to model the propagation of a broadband $\mathrm{THz}$ pulse as it propagates through fibers with a variety of core diameters. The simulations determined that fibers with 140-250 $\mu \mathrm{m}$ cores may be suitable for $\mathrm{CW}$ or pulsed waveguiding in the frequency range of $0.5-3 \mathrm{THz}$. Present growth methods are not able to produce the material quality necessary for effective waveguiding. 


\section{Bibliography}

1. Wolf, S. A. Spintronics: A Spin-Based Electronics Vision for the Future. Science 294, 1488-1495 (2001).

2. Žutić, I., Fabian, J., and Das Sarma, S. Spintronics: Fundamentals and applications. Rev. Mod. Phys. 76, 323-410 (2004).

3. Shull, C. G., Strauser, W. A., and Wollan, E. O. Neutron Diffraction by Paramagnetic and Antiferromagnetic Substances. Phys. Rev. 83, 333-345 (1951).

4. Ando, T., Matsumoto, Y., and Uemura, Y. Theory of Hall Effect in a Two-Dimensional Electron System. J. Phys. Soc. Jpn. 39, 279-288 (1975).

5. Thouless, D. J., Kohmoto, M., Nightingale, M. P., and den Nijs, M. Quantized Hall Conductance in a Two-Dimensional Periodic Potential. Phys. Rev. Lett. 49, 405-408 (1982).

6. Klitzing, K. v., Dorda, G., and Pepper, M. New Method for High-Accuracy Determination of the FineStructure Constant Based on Quantized Hall Resistance. Phys. Rev. Lett. 45, 494-497 (1980).

7. Halperin, B. I. Quantized Hall conductance, current-carrying edge states, and the existence of extended states in a two-dimensional disordered potential. Phys. Rev. B 25, 2185-2190 (1982).

8. Hasan, M. Z. and Kane, C. L. Colloquium: Topological insulators. Rev. Mod. Phys. 82, 3045-3067 (2010).

9. Hosur, P. Circular photogalvanic effect on topological insulator surfaces: Berry-curvature-dependent response. Phys. Rev. B 83, 35309 (2011).

10. Stevens, M. J., Smirl A. L., Bhat R. D. R., Najmaie A., Sipe J. E., and van Driel H. M. Quantum Interference Control of Ballistic Pure Spin Currents in Semiconductors. Phys. Rev. Lett. 90, 136603 (2003).

11. Galanakis, D. and Stanescu, T. D. Electrostatic effects and band bending in doped topological insulators. Phys. Rev. B 86, 195311 (2012).

12. Mishra, S. K., Satpathy, S., and Jepsen, O. Electronic structure and thermoelectric properties of bismuth telluride and bismuth selenide. J. Phys. Condens. Matter 9, 461 (1997).

13. Hor, Y. S., Richardella A., Roushan P., Xia Y., Checkelsky J. G., Yazdani A., Hasan M. Z., Ong N. P., and Cava R. J. p-type Bi2Se3 for topological insulator and low-temperature thermoelectric applications. Phys. Rev. B 79, 195208 (2009).

14. Hsieh, D., Mclver J. W., Torchinsky D. H., Gardner D. R., Lee Y. S., and Gedik N. Nonlinear Optical Probe of Tunable Surface Electrons on a Topological Insulator. Phys. Rev. Lett. 106, 57401 (2011).

15. Kumar, N., Ruzicka B. A., Butch N. P., Syers P., Kirshenbaum K., Paglione J., and Zhao H. Spatially resolved femtosecond pump-probe study of topological insulator Bi2Se3. Phys. Rev. B 83, 235306 (2011).

16. Di Pietro, P., Vitucci F. M., Nicoletti D., Baldassarre L., Calvani P., Cava R., Hor Y. S., Schade U., and Lupi S. Optical conductivity of bismuth-based topological insulators. Phys. Rev. B 86, 45439 (2012). 
17. Valdés Aguilar, R., Stier A. V., Liu W., Bilbro L. S., George D. K., Bansal N., Wu L., Cerne J., Markelz A. G., Oh S., and Armitage N. P. Terahertz Response and Colossal Kerr Rotation from the Surface States of the Topological Insulator Bi2Se3. Phys. Rev. Lett. 108, 87403 (2012).

18. Glinka, Y. D., Babakiray S., Johnson T. A., Bristow A. D., Holcomb M. B., and Lederman D. Ultrafast carrier dynamics in thin-films of the topological insulator Bi2Se3. Appl. Phys. Lett. 103, 151903 (2013).

19. Lu, S., Zhao C., Zou Y., Chen S., Chen Y., Li Y., Zhang H., Wen S., and Tang D. Third order nonlinear optical property of Bi2Se3. Opt. Express 21, 2072-2082 (2013).

20. Mclver, J. W., Hsieh, D., Steinberg, H., Jarillo-Herrero, P., and Gedik, N. Control over topological insulator photocurrents with light polarization. Nat. Nanotechnol. 7, 96-100 (2012).

21. Sim, S., Brahlek M. Koirala N., Cha S., Oh S., and Choi H. Ultrafast terahertz dynamics of hot Dirac-electron surface scattering in the topological insulator Bi2Se3. Phys. Rev. B 89, 165137 (2014).

22. Mclver, J. W., Hsieh D., Drapcho S. G., Torchinsky D. H., Gardner D. R., Lee Y. S., and Gedik N. Theoretical and experimental study of second harmonic generation from the surface of the topological insulator Bi2Se3, Phys. Rev. B 86, 35327 (2012).

23. Zhao, C., Zou Y., Chen Y., Wang Z., Lu S., Zhang H., Wen S., and Tang D. Wavelength-tunable picosecond soliton fiber laser with Topological Insulator: Bi2Se3 as a mode locker. Opt. Express 20, 27888-27895 (2012).

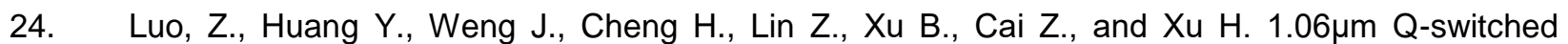
ytterbium-doped fiber laser using few-layer topological insulator Bi2Se3 as a saturable absorber. Opt. Express 21, 29516-29522 (2013).

25. Cao, T. and Wang, S. Topological insulator metamaterials with tunable negative refractive index in the optical region. Nanoscale Res. Lett. 8, 526 (2013).

26. Sun, Y., Cheng H., Gao S., Liu Q., Sun Z., Xiao C., Wu C., Wei S., and Xie Y. Atomically Thick Bismuth Selenide Freestanding Single Layers Achieving Enhanced Thermoelectric Energy Harvesting. J. Am. Chem. Soc. 134, 20294-20297 (2012).

27. Li, J., Jiang F., Yang B., Song X.-R., Liu Y., Yang H.-H., Cao D.-R., Shi W.-R., and Chen G.-N. Topological insulator bismuth selenide as a theranostic platform for simultaneous cancer imaging and therapy. Sci. Rep. 3, (2013).

28. Hutasoit, J. A. and Stanescu, T. D. Induced spin texture in semiconductor/topological insulator heterostructures. Phys. Rev. B 84, (2011).

29. Kane, C. L. and Mele, E. J. A New Spin on the Insulating State. Science 314, 1692-1693 (2006).

30. Moore, J. Topological insulators: The next generation. Nat. Phys. 5, 378-380 (2009).

31. Zhang, T., Cheng P., Chen X., Jia J.-F., Ma X., He K., Wang L., Zhang H., Dai X., Fang Z., Xie X., and Xue Q.-K. Experimental Demonstration of Topological Surface States Protected by Time-Reversal Symmetry. Phys. Rev. Lett. 103, 266803 (2009).

32. Roushan, P., Seo J., Parker C. V., Hor Y. S., Hsieh D., Qian D., Richardella A., Hasan M. Z., Cava R. J., and Yazdani A. Topological surface states protected from backscattering by chiral spin texture. Nature 460, 1106-1109 (2009). 
33. Alpichshev, Z., Analytis J. G., Chu J.-H., Fisher I. R., Chen Y. L., Shen Z. X., Fang A., and Kapitulnik A. STM Imaging of Electronic Waves on the Surface of Bi2Se3: Topologically Protected Surface States and Hexagonal Warping Effects. Phys. Rev. Lett. 104, 16401 (2010).

34. Li, C. H., van 't Erve O. M. J., Robinson J. T., Liu Y., Li L., and Jonker B. T. Electrical detection of charge-current-induced spin polarization due to spin-momentum locking in Bi2Se3. Nat. Nanotechnol. 9, 218-224 (2014).

35. Zhang, Y., He K., Chang C.-Z., Song C.-L., Wang L.-L., Chen X., Jia J.-F., Fang Z., Dai X., Shan W.-Y., Shen S.-Q., Niu Q., Qi X.-L., Zhang S.-C., Ma X.-C., and Xue Q.-K. Crossover of the threedimensional topological insulator Bi2Se3 to the two-dimensional limit. Nat. Phys. 6, 584-588 (2010).

36. Kong, D. Cha J. J., Lai K., Peng H., Analytis J. G., Meister S., Chen Y., Zhang H.-J., Fisher I. R., Shen Z.-X., and Cui Y. Rapid Surface Oxidation as a Source of Surface Degradation Factor for Bi2Se3. ACS Nano. 5, 4698-4703 (2011).

37. Hong, S. S., Cha, J. J., Kong, D., and Cui, Y. Ultra-low carrier concentration and surface-dominant transport in antimony-doped Bi2Se3 topological insulator nanoribbons. Nat. Commun. 3, 757 (2012).

38. Wang, Z., Lin T., Wei P., Liu X., Dumas R., Liu K., and Shi J. Tuning carrier type and density in Bi2Se3 by Ca-doping. Appl. Phys. Lett. 97, 42112 (2010).

39. Zhang, Y., Chang C.-Z., He K., Wang L.-L., Chen X., Jia J.-F., Ma X.-C., and Xue Q.-K. Doping effects of $\mathrm{Sb}$ and $\mathrm{Pb}$ in epitaxial topological insulator Bi2Se3 thin films: An in situ angle-resolved photoemission spectroscopy study. Appl. Phys. Lett. 97, 194102 (2010).

40. Sobota, J. A., Yang S.-L., Kemper A. F., Lee J. J., Schmitt F. T., Li W., Moore R. G., Analytis J. G., Fisher I. R., Kirchmann P. S., Devereaux T. P., and Shen Z.-X. Direct Optical Coupling to an Unoccupied Dirac Surface State in the Topological Insulator Bi2Se3. Phys. Rev. Lett. 111, 136802 (2013).

41. Zhu, L.-G., Kubera, B., Fai Mak, K., and Shan, J. Effect of Surface States on Terahertz Emission from the Bi2Se3 Surface. Sci. Rep. 5, 10308 (2015).

42. Braun, L., Mussler G., Hruban A., Konczykowski M., Schumann T., Wolf M., Münzenberg M., Perfetti L., and Kampfrath T. Ultrafast photocurrents at the surface of the three-dimensional topological insulator Bi2Se3. ArXiv151100482 Cond-Mat (2015).

43. Karch, J., Olbrich P., Schmalzbauer M., Brinsteiner C., Wurstbauer U., Glazon M. M., Tarasenko S. A., Ivchenko E. L., Weiss D., Eroms J., and Ganichev S. D. Photon helicity driven electric currents in graphene. ArXiv10021047 Cond-Mat (2010).

44. Côté, D., Laman, N., and Driel, H. M. van. Rectification and shift currents in GaAs. Appl. Phys. Lett. 80, 905-907 (2002).

45. Nastos, F. and Sipe, J. E. Optical rectification and shift currents in GaAs and GaP response: Below and above the band gap. Phys. Rev. B 74, 35201 (2006).

46. Cook, A. M., Fregoso, B. M., de Juan, F., and Moore, J. E. Design principles for shift current photovoltaics. ArXiv150708677 Cond-Mat (2015).

47. Aversa, C. and Sipe, J. E. Coherent current control in semiconductors: a susceptibility perspective. IEEE J. Quantum Electron. 32, 1570-1573 (1996).

48. Atanasov, R., Haché, A., Hughes, J. L. P., van Driel, H. M., and Sipe, J. E. Coherent Control of Photocurrent Generation in Bulk Semiconductors. Phys. Rev. Lett. 76, 1703-1706 (1996). 
49. van Driel, H. M., Sipe, J. E., Haché, A., and Atanasov, R. Coherence Control of Photocurrents in Semiconductors. Phys. Status Solidi B 204, 3-8 (1997).

50. Costa, L., Betz, M., Spasenović, M., Bristow, A. D., and van Driel, H. M. All-optical injection of ballistic electrical currents in unbiased silicon. Nat. Phys. 3, 632-635 (2007).

51. Haché, A., Kostoulas Y., Atanasov R., Hughes J. L. P., Sipe J. E., and van Driel H. M. Observation of Coherently Controlled Photocurrent in Unbiased, Bulk GaAs. Phys. Rev. Lett. 78, 306-309 (1997).

52. Sun, D., Divin C., Rioux J., Sipe J. E., Berger C., de Heer W. A., First P. N., and Norris T. B. Coherent Control of Ballistic Photocurrents in Multilayer Epitaxial Graphene Using Quantum Interference. Nano Lett. 10, 1293-1296 (2010).

53. Newson, R. W., Ménard, J.-M., Sames, C., Betz, M., and Driel, H. M. van. Coherently Controlled Ballistic Charge Currents Injected in Single-Walled Carbon Nanotubes and Graphite. Nano Lett. 8, 1586-1589 (2008).

54. Laman, N., Bieler, M., and Driel, H. M. van. Ultrafast shift and injection currents observed in wurtzite semiconductors via emitted terahertz radiation. J. Appl. Phys. 98, 103507 (2005).

55. Danishevskii A. M., Kastal'skii A. A., Ryvkin, S. M., and Yaroshetskii I. D. Dragging of Free Carriers by Photons in Direct Interband Transitions in Semiconductors. Sov. Phys. Jetp-Ussr 31, 292- (1970).

56. Obraztsov, P. A., Kaplas T., Gamov S. V., Kuwata-Gonokami M., Obraztsov A. N., and Svirko Y. P. All-optical control of ultrafast photocurrents in unbiased graphene. Sci. Rep. 4, (2014).

57. Plank, H., Golub L. E., Bauer S., Bel'kov V. V., Herrmann T., Olbrich P., Eschbach M., Plucinski L., Kampmeier J., Lanius M., Mussler G., Grützmacher D., and Ganichev S. D. Photon Drag Effect in Bi2Se3 Three Dimensional Topological Insulators. Phys. Rev. B 93, (2016).

58. Nastos, F. and Sipe, J. E. Optical rectification and current injection in unbiased semiconductors. Phys. Rev. B 82, 235204 (2010).

59. Bieler, M., Pierz, K., and Siegner, U. Simultaneous generation of shift and injection currents in (110)-grown GaAs/AlGaAs quantum wells. J. Appl. Phys. 100, 83710 (2006).

60. Brumer, P. and Shapiro, M. Laser Control of Molecular Processes. Annu. Rev. Phys. Chem. 43, 257-282 (1992).

61. Cheng, J. L., Vermeulen, N., and Sipe, J. E. Third-order nonlinearity of graphene: Effects of phenomenological relaxation and finite temperature. Phys. Rev. B 91, 235320 (2015).

62. Rioux, J., Sipe, J. E., and Burkard, G. Interference of stimulated electronic Raman scattering and linear absorption in coherent control. Phys. Rev. B 90, 115424 (2014).

63. Muniz, R. A. and Sipe, J. E. Coherent control of optical injection of spin and currents in topological insulators. Phys. Rev. B 89, 205113 (2014).

64. Bas D. A., Vargas-Velez K., Babakiray S., Johnson T. A., Borisov P., Stanescu T. D., Lederman D., and Bristow A. D. Coherent control of injection currents in high-quality films of Bi2Se3. Appl. Phys. Lett. 106, 41109 (2015).

65. Fu, L. and Kane, C. L. Superconducting Proximity Effect and Majorana Fermions at the Surface of a Topological Insulator. Phys. Rev. Lett. 100, 96407 (2008). 
66. Wang, J., Mabuchi, H. and Qi, X.-L. Calculation of divergent photon absorption in ultrathin films of a topological insulator. Phys. Rev. B 88, 195127 (2013).

67. Seo, J., Roushan P., Beidenkopf H., Hor Y. S., Cava R. J., and Yazdani A. Transmission of topological surface states through surface barriers. Nature 466, 343-346 (2010).

68. Olbrich, P., Golub L. E., Herrmann T., Danilov S. N., Plank H., Bel'kov V. V., Mussler G., Weyrich Ch., Schneider C. M., Kampmeier J., Grützmacher D., Plucinski L., Eschbach M., and Ganichev S. D. Room-Temperature High-Frequency Transport of Dirac Fermions in Epitaxially Grown Sb2Te3- and Bi2Se3-Based Topological Insulators. Phys. Rev. Lett. 113, 96601 (2014).

69. Rowley, J. D., Bas, D. A., Zawilski, K. T., Schunemann, P. G., and Bristow, A. D. Terahertz emission from ZnGeP2: phase-matching, intensity, and length scalability. J. Opt. Soc. Am. B 30, 2882 (2013).

70. Rowley, J. D., Wahlstrand J. K., Zawilski K. T., Schunemann P. G., Giles N. C., and Bristow A. D. Terahertz generation by optical rectification in uniaxial birefringent crystals. Opt. Express 20, 1696816973 (2012).

71. Chiker, F., Abbar, B., Tadjer, A., Aourag, H., and Khelifa, B. Full potential calculation of structural, electronic and optical properties of CdSiP2 and CdGeP2. Mater. Sci. Eng. B 98, 81-88 (2003).

72. Boyd, G., Buehler, E., Storz, F., and Wernick, J. Linear and nonlinear optical properties of ternary AlIBIVC2Vchalcopyrite semiconductors. IEEE J. Quantum Electron. 8, 419-426 (1972).

73. Petrov, V., Noack, F., Tunchev, I., Schunemann, P., and Zawilski, K. The nonlinear coefficient d36 of CdSiP2. Proc. SPIE 7197, 71970M-71970M-8 (2009).

74. Zawilski, K. T., Schunemann P. G., Pollak T. C., Zelmon D. E., Fernelius N. C., and Hopkins F. K. Growth and characterization of large CdSiP2 single crystals. J. Cryst. Growth 312, 1127-1132 (2010).

75. Rowley, J. D., Pierce J. K., Brant A. T., Halliburton L. E., Giles N. C., Schunemann P. G., and Bristow A. D. Broadband terahertz pulse emission from ZnGeP2. Opt. Lett. 37, 788-790 (2012).

76. Exter, M. van, Fattinger, C., and Grischkowsky, D. Terahertz time-domain spectroscopy of water vapor. Opt. Lett. 14, 1128 (1989).

77. Xin, X., Altan, H., Saint, A., Matten, D., and Alfano, R. R. Terahertz absorption spectrum of para and ortho water vapors at different humidities at room temperature. J. Appl. Phys. 100, 94905 (2006).

78. Rowley, J. D. Chalcopyrite Semiconductors as Sources for Terahertz Spectroscopy. (WEST VIRGINIA UNIVERSITY, 2013).

79. Yost, B. T., Cushing S. K., Meng F., Bright J., Bas D. A., Wu N., and Bristow A. D. Investigation of band gap narrowing in nitrogen-doped $\mathrm{La}_{2} \mathrm{Ti}_{2} \mathrm{O}_{7}$ with transient absorption spectroscopy. Phys. Chem. Chem. Phys. 17, 31039-31043 (2015).

80. Ferreira, V. M., Baptista, J. L., Petzelt, J., Komandin, G. A., and Voitsekhovskii, V. V. Loss spectra of pure and La-doped MgTiO3 microwave ceramics. J. Mater. Res. 10, 2301-2305 (1995).

81. Richter, C. and Schmuttenmaer, C. A. Exciton-like trap states limit electron mobility in TiO2 nanotubes. Nat. Nanotechnol. 5, 769-772 (2010).

82. Li, J., Cushing S. K., Meng F., Senty T. R., Bristow A. D., and Wu N. Plasmon-induced resonance energy transfer for solar energy conversion. Nat. Photonics 9, 601-607 (2015). 
83. Tinkham, M. Far Infrared Spectra of Magnetic Materials. J. Appl. Phys. 33, 1248-1253 (1962).

84. Lui, M., Drucker J., King A. R., Kotthaus J. P., Hansma P. K., and Jaccarino V. Observation of antiferromagnetic resonance in epitaxial films of MnF2. Phys. Rev. B 33, 7720-7723 (1986).

85. Xie, M.-Y., Peng X.-N., Fu X.-F., Zhang J.-J., Li G.-L., and Yu X.-F. Synthesis of Yb3+/Er3+ codoped MnF2 nanocrystals with bright red up-converted fluorescence. Scr. Mater. 60, 190-193 (2009).

86. Birgeneau, R. J., Guggenheim, H. J., and Shirane, G. Neutron Scattering Investigation of Phase Transitions and Magnetic Correlations in the Two-Dimensional Antiferromagnets K2NiF4, Rb2MnF4, Rb2FeF4. Phys. Rev. B 1, 2211-2230 (1970).

87. Schulhof, M. P., Heller, P., Nathans, R., and Linz, A. Critical Magnetic Scattering in Manganese Fluoride. Phys. Rev. B 1, 2304-2311 (1970).

88. Cowley, R. A., Buyers, W. J. L., Martel, P., and Stevenson, R. W. H. Magnetic excitations and magnetic critical scattering in cobalt fluoride. J. Phys. C Solid State Phys. 6, 2997 (1973).

89. Moussa, F., Hennion M., Rodriguez-Carvajal J., Moudden H., Pinsard L., and Revcolevschi A. Spin waves in the antiferromagnet perovskite LaMnO3: A neutron-scattering study. Phys. Rev. B 54, 1514915155 (1996).

90. Mays, J. M. Nuclear Magnetic Resonances and Mn-O-P-O-Mn Superexchange Linkages in Paramagnetic and Antiferromagnetic LiMnPO4. Phys. Rev. 131, 38-53 (1963).

91. Jones, E. D. and Jefferts, K. B. Zero-Field Manganese Nuclear Magnetic Resonance in Antiferromagnetic Manganese Fluoride. Phys. Rev. 135, A1277-A1280 (1964).

92. Hämäläinen, K., Kao C.-C., Hastings J. B., Siddons D. P., Berman L. E., Stojanoff V., and Cramer S. P. Spin-dependent x-ray absorption of MnO and MnF2. Phys. Rev. B 46, 14274-14277 (1992).

93. Saitoh, T., Bocquet A. E., Mizokawa T., Namatame H., and Fujimori A. Electronic structure of $\mathrm{La}_{1-\mathrm{x}} \mathrm{Sr}_{\mathrm{x}} \mathrm{MnO}_{3}$ studied by photoemission and x-ray-absorption spectroscopy. Phys. Rev. B 51, 1394213951 (1995).

94. Elliott, R. J. and Thorpe, M. F. The effects of magnon-magnon interaction on the two-magnon spectra of antiferromagnets. J. Phys. C Solid State Phys. 2, 1630 (1969).

95. Macfarlane, R. M. Raman Light Scattering from Excitons and Magnons in Cobalt Fluoride. Phys. Rev. Lett. 25, 1454-1457 (1970).

96. Lockwood, D. J. and Cottam, M. G. The spin phonon interaction in FeF2 and MnF2 studied by Raman spectroscopy. J. Appl. Phys. 64, 5876-5878 (1988).

97. Nishitani, J., Kozuki, K., Nagashima, T. and Hangyo, M. Terahertz radiation from coherent antiferromagnetic magnons excited by femtosecond laser pulses. Appl. Phys. Lett. 96, 221906 (2010).

98. Kampfrath, T., Sell A., Klatt G., Pashkin A., Mährlein S., Dekorsy T., Wolf M., Fiebig M., Leitenstorfer A., and Huber R. Coherent terahertz control of antiferromagnetic spin waves. Nat. Photonics 5, 31-34 (2011).

99. Zhou, R., Jin Z., Li G., Ma G., Cheng Z., and Wang X. Terahertz magnetic field induced coherent spin precession in YFeO3. Appl. Phys. Lett. 100, 61102 (2012). 
100. Jin, Z., Mics Z., Ma G., Cheng Z., Bonn M., and Turchinovich D. Single-pulse terahertz coherent control of spin resonance in the canted antiferromagnet $\mathrm{YFeO}$, mediated by dielectric anisotropy. Phys. Rev. B 87, 94422 (2013).

101. Sänger, I., Pavlov, V., Bayer, M., and Fiebig, M. Distribution of antiferromagnetic spin and twin domains in NiO. Phys. Rev. B 74, 144401 (2006).

102. White, R. M., Freedman, R., and Woolsey, R. B. Theory of the antiferromagnetic-resonance linewidth of MnF2. Phys. Rev. B 10, 1039-1043 (1974).

103. Buyers, W. J. L., Cowley, R. A., Holden, T. M., and Stevenson, R. W. H. Observation of a Localized Magnon in Co Doped MnF2. J. Appl. Phys. 39, 1118-1119 (1968).

104. Darwish, S., Salah, H. K., Abu-Teir, M. M., and Saleh, A. M. Optical investigation of 6A1g(6S) 4T2g (4D) absorption band in magnetic MnF2 and RbMnF3 compounds. Solid State Sci. 11, 1470-1474 (2009).

105. Sell, D. D., Greene, R. L. and White, R. M. Optical Exciton-Magnon Absorption in MnF2. Phys. Rev. 158, 489-510 (1967).

106. Stout, J. W. and Matarrese, L. M. Magnetic Anisotropy of the Iron-Group Fluorides. Rev. Mod. Phys. 25, 338-343 (1953).

107. Heller, P. and Benedek, G. B. Nuclear Magnetic Resonance in MnF2 Near the Critical Point. Phys. Rev. Lett. 8, 428-432 (1962).

108. Erickson, R. A. Neutron Diffraction Studies of Antiferromagnetism in Manganous Fluoride and Some Isomorphous Compounds. Phys. Rev. 90, 779-785 (1953).

109. Fitzsimmons, M. R., Yashar P., Leighton C., Schuller I. K., Nogués J., Majkrzak C. F., and Dura J. A. Asymmetric Magnetization Reversal in Exchange-Biased Hysteresis Loops. Phys. Rev. Lett. 84, 3986-3989 (2000).

110. Higuchi, T. and Kuwata-Gonokami, M. Control of antiferromagnetic domain distribution via polarization-dependent optical annealing. Nat. Commun. 7, 10720 (2016).

111. Wu, S. M., Zhang W., KC A., Borisov P., Pearson J. E., Jiang J. S., Lederman D., Hoffmann A., and Bhattacharya A. Antiferromagnetic Spin Seebeck Effect. Phys. Rev. Lett. 116, 97204 (2016).

112. Moriyasu, T., Wakabayashi, S., and Kohmoto, T. Observation of Antiferromagnetic Magnons and Magnetostriction in Manganese Oxide Using Terahertz Time-Domain Spectroscopy. J. Infrared Millim. Terahertz Waves 34, 277-288 (2013).

113. Belanger, D. P., King, A. R., and Jaccarino, V. Temperature dependence of the optical birefringence of MnF2, MgF2, and ZnF2. Phys. Rev. B 29, 2636-2643 (1984).

114. Okada, K. and Kotani, A. Interatomic and Intra-Atomic Configuration Interactions in Core-Level XRay Photoemission Spectra of Late Transition-Metal Compounds. Tokyo Sugaku Kaisya Zasshi 61, 4619-4637 (1992).

115. Nordblad, P., Lundgren, L., Figueroa, E., Gäfvert, U., and Beckman, O. Critical Behaviour of the Magnetic Susceptibility of MnF 2 Near the Néel Point. Phys. Scr. 20, 105 (1979).

116. Allen, S. J., Loudon, R., and Richards, P. L. Two-Magnon Absorption in Antiferromagnetic MnF2. Phys. Rev. Lett. 16, 463-466 (1966). 
117. Gibbons, D. F. Thermal Expansion Coefficients of Manganese Fluoride. Phys. Rev. 115, 11941195 (1959).

118. Cottam, M. G. and Lockwood, D. J. One-magnon Raman scattering in a $S=5 / 2$ antiferromagnet. Phys. Rev. B 31, 641-643 (1985).

119. Cottam, M. G. and Lockwood, D. J. Light scattering in magnetic solids. (Wiley New York (NY), 1986).

120. Okazaki, A., Turberfield, K. C. and Stevenson, R. W. H. Neutron inelastic scattering measurements of antiferromagnetic excitations in $\mathrm{MnF} 2$ at $4.2^{\circ} \mathrm{K}$ and at temperatures up to the Neel point. Phys. Lett. 8, 9-11 (1964).

121. Oguchi, T. Anisotropy Energy in MnF2 at Low Temperatures. Phys. Rev. 111, 1063-1066 (1958).

122. Darby, M. I. Tables of the Brillouin function and of the related function for the spontaneous magnetization. Br. J. Appl. Phys. 18, 1415 (1967).

123. Kotthaus, J. P. and Jaccarino, V. Temperature dependence of the antiferromagnetic resonance linewidth in MnF2. Phys. Lett. A 42, 361-362 (1973).

124. Holden, T. M., Svensson, E. C., Buyers, W. J. L., Cowley, R. A., and Stevenson, R. W. H. TwoMagnon Scattering of Neutrons by MnF2. J. Appl. Phys. 41, 896-897 (1970).

125. Fleury, P. A. and Loudon, R. Scattering of Light by One- and Two-Magnon Excitations. Phys. Rev. 166, 514-530 (1968).

126. Fleury, P. A., Porto, S. P. S. and Loudon, R. Two-Magnon Light Scattering in Antiferromagnetic MnF2. Phys. Rev. Lett. 18, 658-662 (1967).

127. Bayrakci, S. P., Keller, T., Habicht, K., and Keimer, B. Spin-Wave Lifetimes Throughout the Brillouin Zone. Science 312, 1926-1929 (2006).

128. Chinn, S. R., Zeiger, H. J., and O'Connor, J. R. Two-Magnon Raman Scattering and Exchange Interactions in Antiferromagnetic KNiF3 and K2NiF4 and Ferrimagnetic RbNiF3. Phys. Rev. B 3, 17091735 (1971).

129. Seehra, M. S., Helmick, R. E., and Srinivasan, G. Effect of temperature and antiferromagnetic ordering on the dielectric constants of MnO and MnF 2. J. Phys. C Solid State Phys. 19, 1627 (1986).

130. Siethoff, H. and Ahlborn, K. Debye-temperature-elastic-constants relationship for materials with hexagonal and tetragonal symmetry. J. Appl. Phys. 79, 2968-2974 (1996).

131. Tinkham, M. Paramagnetic Resonance in Dilute Iron Group Fluorides. I. Fluorine Hyperfine Structure. Proc. R. Soc. Lond. Ser. Math. Phys. Sci. 236, 535-548 (1956).

132. Woodfield, B. F., Wilson, M. L., and Byers, J. M. Low-Temperature Specific Heat of La1xSrxMnO3+ס. Phys. Rev. Lett. 78, 3201-3204 (1997).

133. Lees, M. R., Petrenko, O. A., Balakrishnan, G., and McK. Paul, D. Specific heat of Pr0.6(Ca1xSrx)0.4MnO3(0< X< 1). Phys. Rev. B 59, 1298-1303 (1999).

134. Zhang, H., Liu C.-X., Qi X.-L., Dai X., Fang Z., and Zhang S.-C. Topological insulators in Bi2Se3, Bi2Te3 and Sb2Te3 with a single Dirac cone on the surface. Nat. Phys. 5, 438-442 (2009). 
135. Tabor, P., Keenan, C., Urazhdin, S. and Lederman, D. Molecular beam epitaxy and characterization of thin Bi2Se3 films on Al2O3 (110). Appl. Phys. Lett. 99, 13111 (2011).

136. Bansal, N., Kim Y. S., Edrey E., Brahlek M., Horibe Y., lida K., Tanimura M., Li G.-H., Feng T., Lee H.-D., Gustafsson T., Andrei E., and Oh S. Epitaxial growth of topological insulator Bi2Se3 film on Si(111) with atomically sharp interface. Thin Solid Films 520, 224-229 (2011).

137. Xue, L., Zhou P., Zhang C. X., He C. Y., Hao G. L., Sun L. Z., and Zhong J. X. First-principles study of native point defects in Bi2Se3. AIP Adv. 3, 52105 (2013).

138. Côté, D., Fraser, J. M., DeCamp, M., Bucksbaum, P. H., and Driel, H. M. van. THz emission from coherently controlled photocurrents in GaAs. Appl. Phys. Lett. 75, 3959-3961 (1999).

139. Grischkowsky, D., Keiding, S., van Exter, M., and Fattinger, C. Far-infrared time-domain spectroscopy with terahertz beams of dielectrics and semiconductors. J. Opt. Soc. Am. B 7, 2006-2015 (1990).

140. Fraser, J. M., Shkrebtii, A. I., Sipe, J. E., and van Driel, H. M. Quantum Interference in ElectronHole Generation in Noncentrosymmetric Semiconductors. Phys. Rev. Lett. 83, 4192-4195 (1999).

141. Ruppert, C., Thunich S., Abstreiter G., Fontcuberta i Morral A., Holleitner A. W., and Betz M. Quantum Interference Control of Femtosecond, $\mu$ A Current Bursts in Single GaAs Nanowires. Nano Lett. 10, 1799-1804 (2010).

142. Sun, D., Divin C., Rioux J., Sipe J. E., Berger C., de Heer W. A., First P. N., and Norris T. B. Coherent control of ballistic photocurrents in multilayer epitaxial graphene using quantum interference. Nano Lett. 10, 1293-1296 (2010).

143. Park, K., Heremans, J. J., Scarola, V. W., and Minic, D. Robustness of Topologically Protected Surface States in Layering of Bi2Te3 Thin Films. Phys. Rev. Lett. 105, 186801 (2010).

144. Ruppert, C., Lohrenz, J., Thunich, S., and Betz, M. Ultrafast field-resolved semiconductor spectroscopy utilizing quantum interference control of currents. Opt. Lett. 37, 3879-3881 (2012).

145. Hache, A., Sipe, J. E., and van Driel, H. M. Quantum interference control of electrical currents in GaAs. IEEE J. Quantum Electron. 34, 1144-1154 (1998).

146. Sames, C., Ménard, J.-M., Betz, M., Smirl, A. L., and van Driel, H. M. All-optical coherently controlled terahertz ac charge currents from excitons in semiconductors. Phys. Rev. B 79, 45208 (2009).

147. Côté, D., Sipe, J. E., and van Driel, H. M. Simple method for calculating the propagation of terahertz radiation in experimental geometries. J. Opt. Soc. Am. B 20, 1374-1385 (2003).

148. Harrel, S. M., Milot, R. L., Schleicher, J. M., and Schmuttenmaer, C. A. Influence of free-carrier absorption on terahertz generation from ZnTe(110). J. Appl. Phys. 107, 33526 (2010).

149. Zhao, H., Loren, E. J., van Driel, H. M., and Smirl, A. L. Coherence Control of Hall Charge and Spin Currents. Phys. Rev. Lett. 96, 246601 (2006).

150. Bahramy, M. S., King P. D. C., de la Torre A., Chang J., Shi M., Patthey L., Balakrishnan G., Hofmann Ph., Arita R., Nagaosa N., and Baumberger F. Emergent quantum confinement at topological insulator surfaces. Nat. Commun. 3, 1159 (2012). 
151. Bas, D. A., Babakiray S., Lederman D., Bristow A. D., Muniz R. A., and Sipe J. E. Photocurrents in Bi2Se3: bulk versus surface, and injection versus shift currents. ArXiv160201074 Cond-Mat Physics (2016).

152. Dobrovinskaya E. R., Lytvynov L. A., Pishchik V. Sapphire - Material, Manufacturing, Applications. (2009).

153. Sobota, J. A., Yang S., Analytis J. G., Chen Y. L., Fisher I. R., Kirchmann P. S., and Shen Z.-X. Ultrafast Optical Excitation of a Persistent Surface-State Population in the Topological Insulator Bi2Se3. Phys. Rev. Lett. 108, 117403 (2012).

154. Sheik-bahae, M., Said, A. A., and Van Stryland, E. W. High-sensitivity, single-beam n2 measurements. Opt. Lett. 14, 955-957 (1989).

155. Sun, D., Wu Z.-K., Divin C., Li X., Berger C., de Heer W. A., First P. N., and Norris T. B. Ultrafast Relaxation of Excited Dirac Fermions in Epitaxial Graphene Using Optical Differential Transmission Spectroscopy. Phys. Rev. Lett. 101, 157402 (2008).

156. Breusing, M., Kuehn S., Winzer T., Malic E., Milde F., Severin N., Rabe J. P., Ropers C., Knorr A., and Elsaesser T. Ultrafast nonequilibrium carrier dynamics in a single graphene layer. Phys. Rev. B 83, 153410 (2011).

157. Kadi, F., Winzer T., Malic E., Knorr A., Göttfert F., Mittendorff M., Winnerl S., and Helm M. Microscopic Description of Intraband Absorption in Graphene: The Occurrence of Transient Negative Differential Transmission. Phys. Rev. Lett. 113, 35502 (2014).

158. Vignaud, D., Lampin, J. F., and Mollot, F. Two-photon absorption in InP substrates in the $1.55 \mu m$ range. Appl. Phys. Lett. 85, 239-241 (2004).

159. Yang, H., Feng X., Wang Q., Huang H., Chen W., Wee A. T. S., and Ji W. Giant Two-Photon Absorption in Bilayer Graphene. Nano Lett. 11, 2622-2627 (2011).

160. Wang, J., Gu, B., Wang, H.-T., and Ni, X.-W. Z-scan analytical theory for material with saturable absorption and two-photon absorption. Opt. Commun. 283, 3525-3528 (2010).

161. Theeten, J. B., Aspnes, D. E., and Chang, R. P. H. A new resonant ellipsometric technique for characterizing the interface between GaAs and its plasma-grown oxide. J. Appl. Phys. 49, 6097-6102 (1978).

162. Tonouchi, M. Cutting-edge terahertz technology. Nat. Photonics 1, 97-105 (2007).

163. Ulbricht, R., Hendry, E., Shan, J., Heinz, T. F., and Bonn, M. Carrier dynamics in semiconductors studied with time-resolved terahertz spectroscopy. Rev. Mod. Phys. 83, 543-586 (2011).

164. Gallot, G., Jamison, S. P., McGowan, R. W., and Grischkowsky, D. Terahertz waveguides. J. Opt. Soc. Am. B 17, 851 (2000).

165. Jamison, S. P., McGowan, R. W., and Grischkowsky, D. Single-mode waveguide propagation and reshaping of sub-ps terahertz pulses in sapphire fibers. Appl. Phys. Lett. 76, 1987-1989 (2000).

166. Chen, L.-J., Chen, H.-W., Kao, T.-F., Lu, J.-Y., and Sun, C.-K. Low-loss subwavelength plastic fiber for terahertz waveguiding. Opt. Lett. 31, 308 (2006).

167. Lai, C.-H., Hsueh Y.-C., Chen H.-W., Huang Y.-J., Chang H.-C., and Sun C.-K. Low-index terahertz pipe waveguides. Opt. Lett. 34, 3457 (2009). 
168. Wang, K. and Mittleman, D. M. Metal wires for terahertz wave guiding. Nature 432, 376-379 (2004).

169. Ji, Y. B., Kim, S. H., Lee, E. S., Son, J.-H., and Jeon, T.-I. Development of optical fiber-coupled terahertz endoscope. in 2010 IEEE Photonics Society Winter Topicals Meeting Series (WTM) 54-55 (2010).

170. Ballato, J., Hawkins T., Foy P., Stolen R., Kokuoz B., Ellison M., McMillen C., Reppert J., Rao A. M., Daw M., Sharma S., Shori R., Stafsudd O., Rice R. R., and Powers D. R. Silicon optical Fiber. Opt. Express 16, 18675 (2008).

171. Ballato, J., Hawkins T., Foy P., Yazgan-Kokuoz B., McMillen C., Burka L., Morris S., Stolen R., and Rice R. Advancements in semiconductor core optical fiber. Opt. Fiber Technol. 16, 399-408 (2010).

172. Gupta, N., McMillen C., Singh R., Podila R., Rao A. M., Hawkins T., Foy P., Morris S., Rice R., Poole K. F., Zhu L., and Ballato J. Annealing of silicon optical fibers. J. Appl. Phys. 110, 93107 (2011).

173. Sazio, P. J. A., Amezcua-Correa A., Finlayson C. E., Hayes J. R., Scheidemantel T. J., Baril N. F., Jackson B. R., Won D.-J., Zhang F., Margine E. R., Gopalan V., Crespi V. H., and Badding J. V. Microstructured Optical Fibers as High-Pressure Microfluidic Reactors. Science 311, 1583-1586 (2006).

174. Tyagi, H. K., Schmidt, M. A., Prill Sempere, L., and Russell, P. S. Optical properties of photonic crystal fiber with integral micron-sized Ge wire. Opt. Express 16, 17227 (2008).

175. Mehta, P., Healy N., Baril N. F., Sazio P. J. A., Badding J. V., and Peacock A. C. Nonlinear transmission properties of hydrogenated amorphous silicon core optical fibers. Opt. Express 18, 16826 (2010).

176. Ballato, J. and Snitzer, E. Fabrication of fibers with high rare-earth concentrations for Faraday isolator applications. Appl. Opt. 34, 6848 (1995).

177. Naftaly, M. and Miles, R. E. Terahertz time-domain spectroscopy of silicate glasses and the relationship to material properties. J. Appl. Phys. 102, 43517 (2007).

178. Marcuse, D. Loss Analysis of Single-Mode Fiber Splices. Bell Syst. Tech. J. 56, 703-718 (1977).

179. Oskooi, A. F., Roundy D., Ibanescu M., Bermel P., Joannopoulos J. D., and Johnson S. G. Meep: A flexible free-software package for electromagnetic simulations by the FDTD method. Comput. Phys. Commun. 181, 687-702 (2010).

180. Bas, D. A., Cushing, S. K., Ballato, J. and Bristow, A. D. Terahertz Waveguiding in Silicon-Core Fibers. ArXiv13050520 Phys. (2013). 


\section{Appendix A: Terahertz Waveguiding}

The $\mathrm{THz}$ frequency range is useful for various technological and scientific applications. ${ }^{162,163} \mathrm{THz}$ radiation lies between the microwave and IR regions of the electromagnetic spectrum and so $\mathrm{THz}$-based technologies share many of the same potential applications as its spectral neighbors. However, THz components are less developed and so many hurdles exist that must be overcome in order to develop suitable device architectures. For example, and most simply, high-quality transmission fibers are needed to confine and direct $\mathrm{THz}$ radiation in a manner that is robust and enabling of compact practical devices. Various schemes have been investigated for $\mathrm{THz}$ waveguiding using either dielectric ${ }^{164-167}$ and metallic ${ }^{168}$ materials. Dielectric fibers provide advantages in length and flexibility, extend low-frequency waveguiding capabilities, and have much lower power absorption than metal waveguides. ${ }^{165}$ Existing schemes are typically very efficient for laboratory use in the specific applications for which they are designed, with attenuation coefficients as low as about $0.01 \mathrm{~cm}^{-1} .{ }^{164,166}$ However, designing a multipurpose, commerciallymanufactured $\mathrm{THz}$ waveguide would further $\mathrm{THz}$ technology and its applications. Flexibility and large available wavelength ranges are essential for successful applications such as endoscopy, ${ }^{169}$ where the waveguide's core can be protected by a cladding the same way as in optical fibers.

It has been predicted that high purity silicon would be an optimal material for $\mathrm{THz}$ applications. Factors that support the claim are its relatively constant refractive index and low absorption over the $\mathrm{THz}$ frequency range, ${ }^{139}$ as well as its high thermal conductivity, high optical damage threshold, and low-loss transmission in the far-IR frequency range. ${ }^{170}$ Ballato, et al., have manufactured various silicon-core fibers using a scalable molten-core technique, which shows promising results for future applications. ${ }^{170-172}$ Alternative manufacturing techniques include chemical vapor deposition ${ }^{173}$ and high pressure melt infiltration. ${ }^{174}$ The molten-core method is particularly appropriate for $\mathrm{THz}$ applications, because it provides for large core $(>50 \mu \mathrm{m}$ ) fiber fabrication (potentially single-mode at $\mathrm{THz}$ frequencies) with length scalability and potentially high-speed manufacturing.

Silicon-core optical fibers have been shown to guide near- and mid-IR light, increase nonlinearoptical properties ${ }^{175}$ and act as Faraday isolators. ${ }^{176}$ The purpose of the work in this section is to extend the 
range of potential applications for silicon optical fiber to $\mathrm{THz}$ waveguides. The propagation of pulsed $\mathrm{THz}$ radiation is simulated to capture the propagation effects over a wide tuning range for either narrow band CW or broadband pulse transmission.

\section{A.1: Mode analysis}

Similarly to conventional optical fibers, semiconductor-core waveguides for $\mathrm{THz}$ applications operate on the (classical) principal of total-internal reflection. The radiation modes are confined and guided by the higher index silicon core $\left(n_{\text {core }}=3.418\right.$ at $\left.\sim 1 \mathrm{THz}\right),{ }^{139}$ which is clad by silica $\left(n_{\text {clad }}=1.954\right.$ at $\sim 1 \mathrm{THz}) .{ }^{139,177}$ Optical fibers are characterized by a normalized frequency parameter $V=\pi(N A) d / \lambda$, where the core diameter, $d$, is taken for the purposes of this work to be $140,170,200$, and $250 \mu \mathrm{m}$. The wavelength $\lambda$, is defined as $c / v n_{\text {core }}$, with $c$ being the vacuum light speed and the frequency range $v=0.5-3 \mathrm{THz}$, which correspond to core wavelengths of $\sim 29.2 \mu \mathrm{m}-\sim 175 \mu \mathrm{m}$. The numerical aperture for an optical fiber is given by $\mathrm{NA} \approx \sin \theta_{c}=\sqrt{n_{\text {core }}^{2}-n_{\text {clad }}^{2}} / n_{0}$, where $\theta_{c}$ is the half acceptance angle and $n_{0}$ for the external medium, which is usually air. The core-cladding refractive index contrast is $>1$, which leads to a very high NA compared to conventional optical fibers, where the refractive index contrast is typically $\sim 10^{-3}$. For practical considerations, such as for single-mode operation, to eliminate intermodal dispersion, and to increase output efficiency, it is preferable to minimize $V$, hence NA. This can be accomplished by setting $n_{0}=3.418$ to simulate end-butt coupling to a silicon chip or hemispherical lens.

$V$ is graphed as a function of frequency in Figure A.1(a), showing a linear increase in $V$ with frequency and a steeper slope with increasing core diameter. A fiber is single mode if its normalized frequency is less than the first root of the $J_{0}$ Bessel function, i.e. $V<2.405$, which is indicated as the horizontal line on the plot. Based on the normalized frequency calculations, it was determined that for $d<140 \mu \mathrm{m}$, wave propagation will have too much spatial overlap into the cladding, leading to higher attenuation. On the other hand, for $d>140 \mu \mathrm{m}$ the wavelength range for single-mode operation diminishes. 

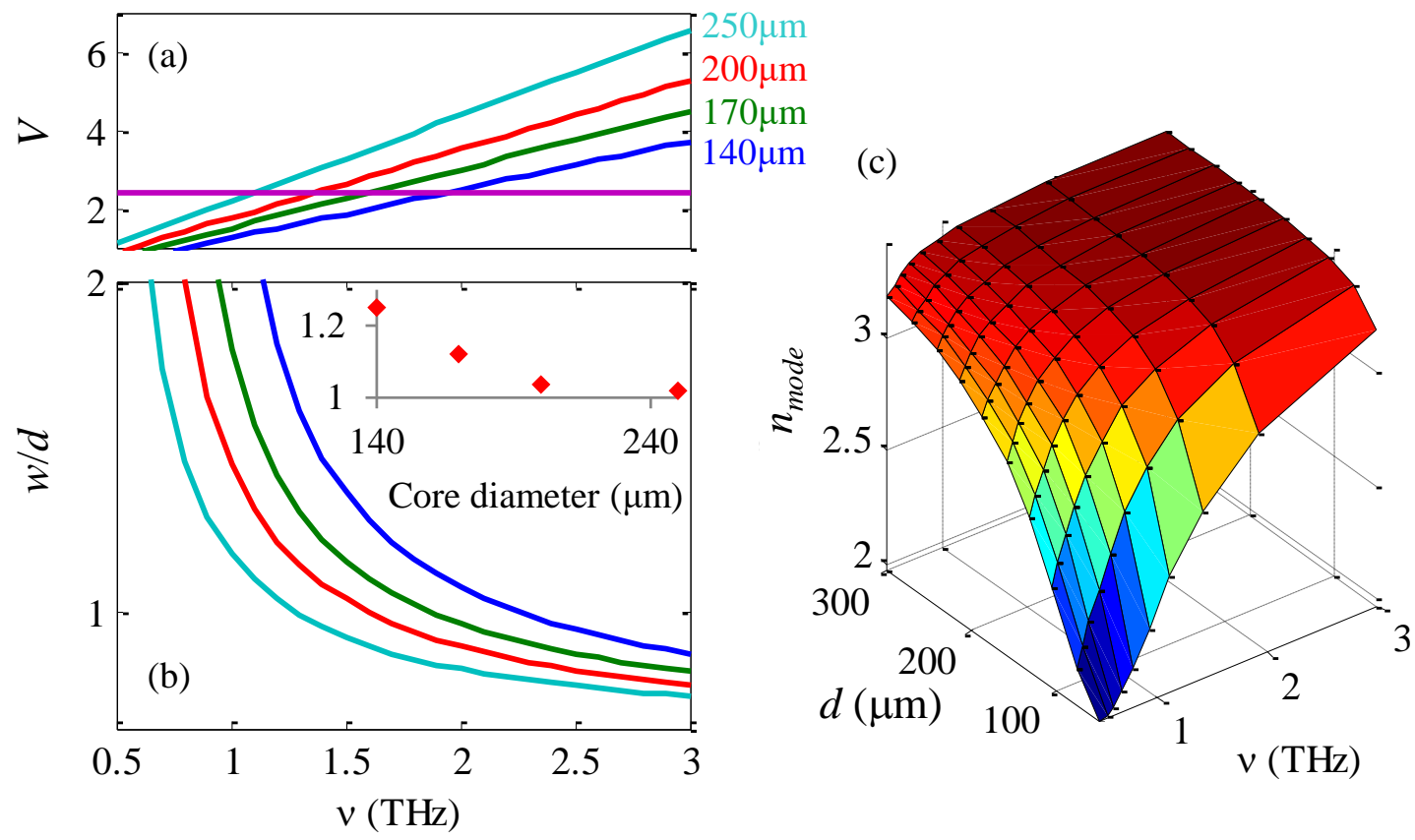

Figure A.1: (a) Normalized frequency parameter $V$ for fibers with $d=140,170,200$, and 250 $\mu \mathrm{m}$ (blue, green, red, and cyan). The horizontal line is at $V=2.405$. (b) The normalized $1 / e^{2}$ diameter of fundamental $\mathrm{HE}_{11}$ mode. Inset shows simulated Gaussian pulse values of $w / d$ versus core thickness. (c) Modal refractive index THz frequency and core diameters.

Using commercially available OptiMode software, the fundamental $\mathrm{HE}_{11}$ guided mode profile was computed. The $1 / e^{2}$-diameter of the mode profile was found in terms of the normalized frequency parameter and core diameter ${ }^{178}$ as

$$
w=d\left(0.65+1.619 V^{-1.5}+2.879 V^{-6}\right) .
$$

The normalized mode profile $w / d$ is plot as a function of frequency in Figure A.1(b). The $w / d$ parameter increases geometrically with decreasing frequency, but more slowly for wider core diameters. For high frequencies, $v>1.5 \mathrm{THz}, w / d$ ranges from $0.75-1.4$. Values of $w / d>>1$ leads to overlap of the mode in the cladding, which for $\mathrm{THz}$ radiation will result in absorption in the silica. ${ }^{139,177}$ This issue is limited for wider cores, but even the $250 \mu \mathrm{m}$ core shows overlap into the silica.

The modal index $n_{\text {mode }}$ was determined from the spatial extent of the mode profile; see Figure A.1(c). It is found that for high frequencies and large core diameters $n_{\text {mode }}$ approaches the value of bulk silicon, $n_{\text {core }}$. At low frequencies and especially in smaller core fibers there is increased mode overlap with 
the cladding and the mode index approaches $n_{\text {clad }}$, confirming the result of the normalized $1 / e^{2}$-diameter of the mode profile.

Fresnel losses occur at the interfaces between external Si (chip or hemisphere) and fiber, due to mismatch between the $n_{\text {mode }}$ and $n_{0}$. For example, $0.5-\mathrm{THz}$ radiation coupling to the $140-\mu \mathrm{m}$ fiber has a reflection of $I_{R} / I_{0}=\left(n_{S i}^{2}-n_{\text {mode }}^{2}\right) /\left(n_{S i}^{2}+n_{\text {mode }}^{2}\right) \approx 0.03$. Reflection decreases as the core diameter and frequency increase, because $n_{\text {mode }} \rightarrow n_{0}$.

\section{A.2: Finite-difference time-domain simulations}

Finite-difference time-domain (FDTD) simulations also were performed on fibers with core diameters of $d=140,170,200$, and $250 \mu \mathrm{m}$, and cladding outer diameters of $1.6 \mu \mathrm{m}$ using open-source Meep software. ${ }^{179}$ Due to the radial symmetry of the optical fiber geometry two-dimensional simulations are sufficient to determine propagation trends. The simulation cell has a grid size of $5000 \mu \mathrm{m} \times 3000 \mu \mathrm{m}$, surrounded by a $100-\mu \mathrm{m}$ border to absorb all reflections into the simulation region. The spatial and temporal resolution is set to $6.25 \mu \mathrm{m}$ and $41.7 \mathrm{fs}$ respectively.

The core is centrally located inside the cell with dimensions of $4000 \mu \mathrm{m} \times d$ and symmetric cladding has dimensions of $4000 \mu \mathrm{m} \times 1600 \mu \mathrm{m}$. A bulk Si region is placed at the entrance and exit regions of the fiber region to mimic either end-butt coupled focusing hemispheres or planar chip. The dielectric constants are determined from fitting the literature results ${ }^{139}$ with a damped oscillator model to determine the dispersion relation

$$
\varepsilon(\omega)=\varepsilon(\infty)+\frac{A \omega_{0}^{2}}{\omega_{0}^{2}-\omega^{2}-i \omega \gamma},
$$

where the high-frequency dielectric constant is $\varepsilon(\infty)=11.67895$, the oscillator amplitude is $A=0.00248$, resonance frequency is $\omega_{0}=2.35368 \mathrm{THz}$ and damping rate is $\gamma=17.1509 \mathrm{THz}$ for the Si core. Similarly, $\varepsilon(\infty)=3.64407, A=0.16585, \omega_{0}=5.47686 \mathrm{THz}$ and $\gamma=2.96159 \mathrm{THz}$ for the $\mathrm{SiO}_{2}$ cladding. These units are scaled to dimensionless parameters to run in the FDTD code.

The source is described as the discrete time derivative of a Gaussian ${ }^{179}$ waveform as 


$$
E(t)=(-i \omega)^{-1} \frac{\partial}{\partial t} \exp \left(-i \omega t-\frac{\left(t-t_{0}\right)^{2}}{2 w^{2}}\right)
$$

which corresponds to a plane-wave source with a Gaussian envelope determined from the frequencydomain properties of a desired pulse with center frequency of $1.755 \mathrm{THz}$ and the full-width at half-maximum (FWHM) of $2.925 \mathrm{THz}$. These parameters give a pulse width of $\tau_{p} \approx 342 \mathrm{fs}$. This pulse shape approximately reproduces those found by OR. ${ }^{75}$ The input extends across the full input (left) side of the cell, such that the radiation couples to both the core and cladding. This assumes that the mode is nearly equivalent to being at the beam waist if it were a focused Gaussian mode, and also provides a faster travelling reference pulse in the cladding for comparison to the core region of the fiber. From the source radiation, the algorithm incrementally evolves over finite time intervals using Maxwell's equations, ${ }^{178}$ propagating in the $+z$ direction. The FDTD simulation output electric field snapshots separated by about $300 \mathrm{fs}$ in time.

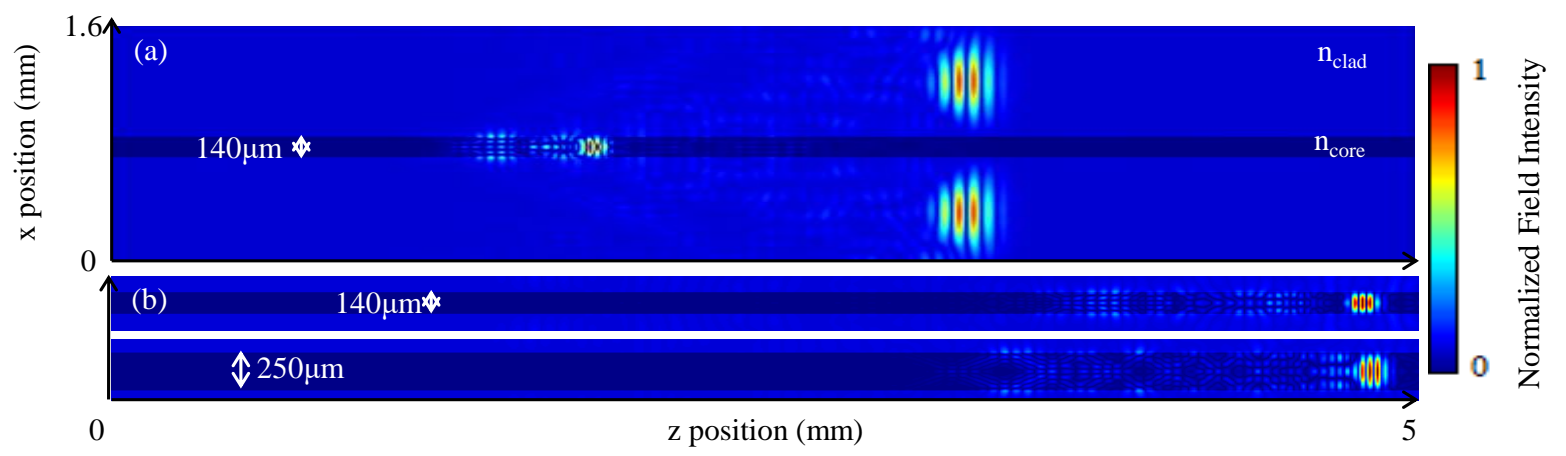

Figure A.2: (a) Spatial pattern of intensity at a single instant in time for a pulse travelling through a $140 \mu \mathrm{m}$ core fiber. Larger darkened areas are a visual aid to show the cladding and should not be confused with a nonzero intensity. (b) Comparison of main pulse and dispersive low-frequency tail for smallest and largest core diameters.

Figure A.2(a) shows the full-cell intensity snapshot at a single time step (17.67 ps after the wave enters the fiber) for $d=140 \mu \mathrm{m}$. Regardless of the core diameter, the bulk of the pulse propagating in the core has a speed of $0.29 c$, which is consistent with $n_{\text {core }}=3.45$. Lower frequency components clearly lag the main pulse at a slower velocity, which is consistent with the determination of $V$. In comparison, the cladding propagation speed is $\sim 0.6 c$, due to the lower index. Hence, core waveguiding can be separated from cladding propagation by taking into account the refractive index contrast between the cladding and the fundamental waveguiding mode. For example, in a $1 \mathrm{~cm}$ long large diameter fiber (for $n_{\text {mode }} \approx n_{\text {core }}$ ), 
the pulses that travel in the fundamental mode and in the cladding should be separated in time by $z / c\left(n_{\text {mode }}-n_{\text {clad }}\right)=48.8 \mathrm{ps}$. This temporal separation is an advantage for time-domain $\mathrm{THz}$ spectroscopy, which could easily identify each component.

A side-by-side comparison of the pulse near the exit of the smallest and largest core diameters at the same time step is shown in Figure A.2(b). A clear tail is present for the lower frequency components in both fibers. In the $140 \mu \mathrm{m}$ fiber the tail is dominated by poorer mode confinement, while in the $250 \mu \mathrm{m}$ fiber intermodal dispersion is more prominent, although both effects are present in each fiber.

The $1 / e^{2}$ diameter of the mode is extracted by examining the transverse position $x$ extracted from the intensity profile for a given time slice, wherein the $z$ position is selected to be the peak $\mathrm{THz}$ intensity. Figure A.3(a) shows the extracted intensity profiles for various positions $z=836,2010,3146$ and $4305 \mu \mathrm{m}$. Shortly after the pulse enters the core, its profile is very narrow and well-confined to the core, but after travelling approximately $4 \mathrm{~mm}$ it fills out the core and the shape becomes relatively stable.
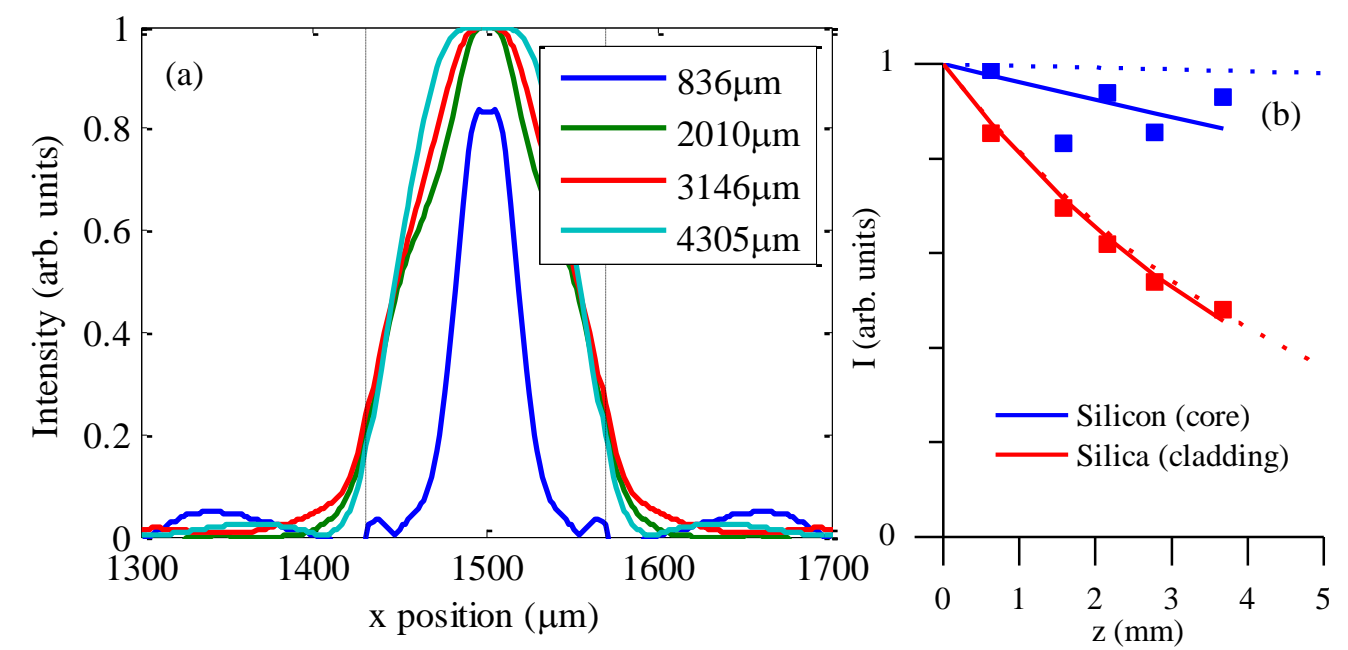

Figure A.3: (a) Evolving shape of the pulse as the peak electric field reaches z-distances given in the legend. Vertical dashed lines indicate the core-cladding boundary. (b) Attenuation of core (blue) and cladding (red) modes. The blue squares represent the flux through the core, and the red squares represent the flux through the entire fiber. Blue and red solid lines are exponential fits to the data points. Blue and red dotted lines are Beer's law attenuation curves for pure silicon and silica, ${ }^{139,176}$ respectively.

When the profile is stable, a $w / d$ value representing the mode confinement can be extracted from a Gaussian fit of the pulse. Mode confinement for all four simulated core diameters is shown in the inset of 
Figure A.1(b). The behavior is consistent with that prediction from Marcuse's equation ${ }^{178}$ showing that smaller core diameters will experience the poorest mode confinement; see Equation A.1. Note that these values are integrated over the pulse spectrum.

Attenuation for a single frequency $(1 \mathrm{THz})$ in the $140 \mu \mathrm{m}$ core fiber was obtained by tracking the simulated flux through planes at several $z$ distances, as shown in Figure A.3(b). Intensity was integrated over the fiber core and compared with the attenuation for pure silicon. The core intensity is attenuated more strongly than would occur in silicon because $n_{\text {mode }}$ has a significant contribution from the cladding refractive index at $1 \mathrm{THz}$; see Figure A.1(c). Intensity integrated over the entire fiber was also compared with the attenuation for pure silica. Attenuation through the fiber cladding is fit well with Beer's law for pure silica.

\section{A.3: Conclusion}

The transmission of a broadband THz pulse through silica-clad, silicon-core optical fibers of various core diameters has been simulated in order to demonstrate the feasibility of this potentially practical approach. ${ }^{180}$ It has been determined that optimal fiber core diameters will fall approximately in the 140$250 \mu \mathrm{m}$ range; ideally suited for the molten-core fabrication approach to semiconductor optical fibers. For smaller diameters, the guided modes will experience more spatial overlap with the cladding, especially for the lower frequency components. For larger diameters, the wavelength range for which the fibers are singlemode is narrower, requiring intermodal dispersion to be taken into account.

FDTD simulations agree well with a quantitative Beer's law absorption analysis, and with normalized frequency calculations and Marcuse's mode-confinement equation. The simulations demonstrate that $\mathrm{THz}$ waveguiding is achievable in semiconductor-core optical fibers of the stated core diameters.

Overall silicon-core optical fibers that can be created using a molten-core pulling technique are suitable for narrowband $\mathrm{CW}$ or possibly broadband pulsed $\mathrm{THz}$ radiation in the range of $0.5-3 \mathrm{THz}$. In practice the fiber-manufacturing method can be altered to use a wider range of materials beyond silicon and silica to improving $\mathrm{THz}$ waveguiding. Since the mode and basic propagation analysis are discussed in 
this section, the experimental proof-of-concept is left to be addressed in future works. Current growth methods are unable to create fibers of high enough quality for effective transmission. 